\title{
Traditionen, Innovationen und Technologietransfer - zur Herstellungstechnik und Funktion älterbronzezeitlicher Schwerter in Niedersachsen ${ }^{1}$
}

\author{
von Jan-Heinrich Bunnefeld, Göttingen, und Stefan Schwenzer, Berlin
}

\begin{abstract}
Bronzezeit; Niedersachsen; Nordischer Kreis; Schwerter; Vollgriffdolche; Metallhandwerk; Technologietransfer; Metallanalysen; Röntgenuntersuchungen; Computertomographie; Gebrauchsspuren.

Age du Bronze; Basse-Saxe; Bronze nordique; épées; poignards à manche massif; artisanat du métal; transfert de technologies; analyse de métaux; radioscopies; scanographie; traces d'utilisation.

Bronze Age; Lower Saxony; Nordic Bronze Age; swords; metal-hilted daggers; metal craft; technology transfer; metal analyses; X-ray examinations; computed tomography; use-wear analyses.
\end{abstract}

Im Rahmen eines durch das Land Niedersachsen finanzierten Projektes wurden makro- und mikroskopische Untersuchungen, Röntgenuntersuchungen, 3D-Computertomographien sowie Metallanalysen (Spurenelement- und Bleiisotopenanalysen) zu niedersächsischen Schwertern der älteren Bronzezeit angefertigt. Die Auswertung ergibt eine Reihe neuer Erkenntnisse, unter anderem wurde an zwei nordischen Vollgriffschwertern eine ungewöhnliche Verzierungstechnik der Knaufplatte durch eingesetzte Stifte festgestellt. Die Untersuchungen bestätigen zudem die Annahme, dass die nordischen Vollgriffschwerter und die aus dem südlichen Mitteleuropa stammenden Achtkantschwerter von den Trägern unterschiedlicher Handwerkstraditionen hergestellt wurden. Bemerkenswert ist, dass sich bei nordischen Vollgriffschwertern in begrenztem Umfang Hinweise auf die Adaption der süddeutschen Guss-, vor allem aber Schäftungstechnik finden, womit sich komplexe Beziehungen und ein selektiver Technologietransfer zwischen den beiden Handwerkstraditionen abzeichnen. Zudem kann belegt werden, dass handwerkliche Traditionen der frühbronzezeitlichen Vollgriffdolche in der Technik der mittelbronzezeitlichen Vollgriffschwerter fortleben. Für die Achtkantschwerter ist eine Produktion in mehreren kleineren Werkstätten anzunehmen. Die schon länger postulierten Werkstattkreise der nordischen Vollgriffschwerter konnten aufgrund der geringen Datenbasis leider nicht nach technischen Kriterien überprüft werden. Die Distribution von Achtkantschwertern in den Norden kann durch Import von süddeutsch verzierten Stücken sowie unverzierten Schwertern, die im Norden eigenhändig verziert wurden, erklärt werden. Dabei kam es zu gegenseitigen Kontakten und Beeinflussungen. Neben ibrer primären Funktion als Waffen ist den Schwertern zweifellos auch soziale und symbolische Bedeutung beizumessen.

Dans le cadre d'un projet financé par le Land de Basse-Saxe, des épées de cette région appartenant au Bronze ancien furent soumises à des examens macro- et microscopiques, à des radioscopies, des scanographies $3 D$ ainsi qu'à des analyses de métaux (analyses des éléments traces et des isotopes du plomb). L'étude livre une série de nouveaux résultats, dont l'identification, d'une technique de décor à l'aide de clous fixés dans la plaque du pommeau de deux épées nordiques à poignée massive. Ces examens confirment en outre l'idée que les épées nordiques à poignée massive et les épées à poignée octogo-

1 Das Projekt „Technische Untersuchungen an älterbronzezeitlichen Schwertern Niedersachsens“ wurde dankenswerterweise im Jahre 2006 vom Niedersächsischen Ministerium für Wissenschaft und Kultur gefördert. Geleitet wurde es von Prof. Dr. K.-H. Willroth (Göttingen), durchgeführt von Dr. S. Schwenzer (Berlin) und ausgewertet zu großen Teilen von J.-H. Bunnefeld M. A. (Göttingen). Ein großer Dank geht an folgende Museen (in alphabetischer Reihenfolge) Museum Burg Bederkesa, Focke Museum Bremen, BachmannMuseum Bremervörde, Niedersächsisches Landesmuseum Hannover, Museum Nienburg/Weser, Schloss Gottorf - Archäologisches Landesmuseum Schleswig, Schwedenspeicher-Museum Stade und Museum Schloss Schönebeck in Vegesack sowie an ihre Mitarbeiter für die freundliche Zusammenarbeit bei der Materialaufnahme. Zahlreiche Röntgenbilder wurden freundlicherweise vom RömischGermanischen Zentralmuseum in Mainz zur Verfügung gestellt. Weitere konnten durch das freundliche Entgegenkommen von Airbus S. A. S. in Bremen und Yxlon International in Hamburg aufgenommen werden. Die Computertomographien konnten bei der Volkswagen AG in Wolfsburg durchgeführt werden. Allen Beteiligten gebührt dafür unser herzlicher Dank. Die archäometallurgischen Untersuchungen wurden am Curt-Engelhorn-Zentrum für Archäometrie in Mannheim vorgenommen. Für Ratschläge und Hilfe bei der Auswertung der Metallanalysen möchten wir uns bei Prof. Dr. E. Pernicka (Tübingen/Mannheim) und Mag. Ing. Mathias Mehofer (Wien) herzlich bedanken. Auch all denen, die nicht namentlich genannt werden konnten, danken wir für ihre Ratschläge und Hilfe! 
nale du sud de l'Europe centrale furent fabriquées par des artisans imprégnés de traditions distinctes. Il faut remarquer que certaines épées nordiques présentent quelques caractéristiques propres aux techniques du coulage du bronze et surtout de l'emmanchement de l'Allemagne du Sud, révélant ainsi des liens complexes et un transfert technologique sélectif entre les deux traditions artisanales. On a également pu établir que les traditions artisanales des poignards à manche massif du Bronze ancien perdurent à travers la technique de fabrication des épées à poignée massive du Bronze moyen. Une production dans plusieurs petits ateliers peut être envisagée pour les épées à poignée octogonale. Vu la faible quantité de données, il fut impossible de vérifier selon des critères techniques les groupes d'ateliers des épées nordiques à poignée massive, postulés déjà depuis longtemps. La distribution des épées à poignée octogonale dans le nord peut s'expliquer par l'importation d'exemplaires décorés à la façon allemande méridionale, comme par celle d'épées nues, qui furent alors décorées par les artisans nordiques locaux. Des contacts et influences réciproques accompagnèrent ces échanges. Outre leur fonction première de servir d'arme, les épées devaient certainement revêtir une signification sociale et symbolique.

In a project funded by the German state of Lower Saxony, macro- and microscopic analyses, X-ray examinations, 3D computed tomography and metal analyses (trace element analyses and lead isotope analyses) were carried out on Early Bronze swords from Lower Saxony. The evaluation provides some new insights, including the discovery of an exceptional ornamental technique involving inserted bolts on the pommels of two Nordic full-hilted swords. Furthermore, the investigations confirm the theory that Nordic full-hilted swords and Southern Central European octagonal hilted swords were produced by craftsmen of different traditions. Remarkably, Nordic full-hilted swords bear evidence of a limited adaptation of casting and mounting techniques of Southern Central European origin. This sheds light on complex relations and a selective technology transfer between these two different craft traditions. In addition, it can be shown that some craft traditions of the Early Bronze metal-hilted daggers live on in some full-hilted swords of later times. The octagonal hilted swords were probably produced in several small workshops. The long-postulated workshop trading areas of Nordic full-hilted swords could unfortunately not be reassessed according to technological criteria because of the scarce data available. The distribution of octagonal hilted swords in the north may be explained by the import of swords with "southern" ornamentation and other non-decorated swords which were finished in the north. This led to reciprocal contact and influences. Besides their primary function as a weapon, the swords would certainly also have carried social and symbolic meanings.

\section{Einleitung}

Die Schwerter der Bronzezeit gehören zu den am häufigsten untersuchten Objekten der Urgeschichte. Untersuchungen speziell zu ihrer Technik wurden dabei jedoch bemerkenswert selten durchgeführt und publiziert. Wichtige Arbeiten für die ältere Bronzezeit im mitteleuropäischen Raum stammen von D. Ankner ${ }^{2}$, H. Drescher ${ }^{3}$, J. Driehaus $^{4}$, H.-J. Hundt ${ }^{5}$ und aus jüngerer Zeit von M. Mödlinger ${ }^{6}$ sowie H. Wüstemann ${ }^{7}$, wobei nur Ankner, Mödlinger und Wüstemann größere Objektserien veröffentlicht haben.

Im vorliegenden Aufsatz soll die Herstellungstechnik und Funktion älterbronzezeitlicher Schwerter (Per. I b bis Per. III) im Gebiet des heutigen Niedersachsen untersucht werden. Dazu wurden 121 Schwerter ausgewählt und katalogisiert. Davon konnten 69 Fundstücke makro- und mikroskopisch begutachtet werden, für 24 Schwerter liegen Röntgenbilder vor und 54 Stücke wurden zum Teil mehrfach beprobt und metallanalytisch untersucht. Sechs Schwerter konnten außerdem dank der freundlichen Unterstützung der Volkswagen AG dreidimensional computertomographisch untersucht werden. In den vorliegenden Kata-

2 Ankner 1977.

3 Drescher 1957; 1958; 1961; 1978.

4 Driehaus 1961; 1968.

5 Hundt 1962; 1965; 1979.

6 Mödlinger 2007; 2011.

7 Wüstemann 1999; 2004. log wurden nur Fundstücke aufgenommen, die tatsächlich untersucht werden konnten.

Das Ziel der Studie ist die Rekonstruktion handwerklicher Tätigkeiten, möglicher Werkstattkreise und die Ermittlung jener Handels- und Kommunikationswege, die den Austausch handwerklicher Fähigkeiten ermöglichten. Ein wichtiger Aspekt dabei ist die Frage, inwiefern die unterschiedlichen Traditionen der frühbronzezeitlichen Vollgriffdolche die spätere Entwicklung beeinflusst haben. Zudem soll die Analyse von Gebrauchsspuren Hinweise auf die Funktion der Schwerter ergeben.

Das heutige Niedersachsen ist für diese Fragestellungen besonders geeignet, da es in der älteren Bronzezeit einen Schnittpunkt zwischen den Handwerkstraditionen des Nordischen Kreises und Süddeutschlands bildet und deshalb Aussagen zu ihrem Nebeneinander und ihren Beziehungen ermöglicht.

\section{Herstellungstechnik}

\section{Griffplatten- und Griffzungenschwerter}

Der Guss ${ }^{8}$ der Griffplatten- und Griffzungenschwerter erfolgte wahrscheinlich in zweiteiligen Gussformen ${ }^{9}$ aus

8 Zum Bronzeguss allgemein siehe u.a. Armbruster 2000; Jantzen 2008.

9 Der Terminus „zweiteilige Gussform“ bedeutet im Falle keramischer Formen nicht unbedingt, dass sie mehrfach verwend- 
Keramik, Bronze oder Stein. Da die Gussnähte sicherlich im Bereich der Schneiden gelegen haben und im Zuge der Nachbearbeitung entfernt wurden, sind sie nicht mehr nachzuweisen. Um die Gussform möglichst rasch vollständig mit der Gussspeise zu füllen, waren die Gießer auf die Schwerkraft und den hydrostatischen Druck angewiesen. Nach dem Zustand erhaltener Gusszapfen zu urteilen, standen Gussformen gerade oder leicht geneigt. Möglicherweise wurden sie mit Sand bzw. Steinen abgestützt oder eingegraben, um ein sich Öffnen zu verhindern und eine stabile Position zu garantieren ${ }^{10}$. Die Gase konnten nicht immer rechtzeitig vor dem Erkalten des Metalls entweichen und bildeten deshalb oft Blasen, die häufig in der Literatur im Sinne der technischen Terminologie nicht ganz korrekt als ,Lunker' bezeichnet werden ${ }^{11}$. Die Position des Angusses lässt sich bei vielen Schwertern über die Verteilung dieser Gasblasen ermitteln ${ }^{12}$.

Bei den Griffplattenschwertern von Buxtehude-Altkloster (Nr. 5) und Hambostel (Nr. 10) zeigen sich im Klingenbereich zahlreiche Blasen. Beim Stück von Hambostel scheint die Griffplatte blasenfrei zu sein, was eher auf einen Anguss an der Klingenspitze hindeutet. An dem Griffzungenschwert aus der „Umgebung von Stade“ (Nr. 39) ist im oberen Klingenbereich auf der Mittelrippe ein größerer Gussfehler aufgetreten, so dass noch einmal nachgegossen werden musste (Abb. 1). Die Griffzungenschwerter von Harsefeld (Nr. 34) und Nindorf (Nr. 36) zeigen im Röntgenbild unterschiedliche Befunde. Das Stück von Nindorf weist abgesehen von einigen kleinen Blasen im Heftbereich nur sehr wenige Hohlräume auf, wohingegen das Schwert von Harsefeld insbesondere im oberen Klingenbereich und im Heft von vielen Blasen durchzogen ist (Abb. 2). Die Befunde sprechen daher für einen Anguss an der Griffzunge. Dies entspricht den Ergebnissen von M. Mödlinger für Griffzungenschwerter in Österreich ${ }^{13}$.

Nach dem Guss wurden die Stücke nachbearbeitet, wobei Gusszapfen und -nähte durch Abschlagen mit einem Meißel oder Schleifen entfernt, Nietlöcher eingefügt und die Schneiden gedengelt wurden. Während des Dengelns musste das Metall immer wieder kurze Zeit zwischengeglüht werden, um das Gefüge zu entspannen, die Sprödigkeit zu reduzieren und Rissen vorzubeugen. Dabei kam es jedoch nicht $\mathrm{zu}$ einer Homogenisierung des Materials ${ }^{14}$.

bar waren und ist daher nicht als Gegenteil zum Wachsausschmelzverfahren zu sehen. Unterschieden werden können sie durch ihre Herstellungsweise. Zweiteilige Gussformen wurden wohl häufig einfach von einem vorhandenen Objekt abgeformt, müssen jedoch nach dem Zusammensetzen nicht ohne Zerstörung zu öffnen gewesen sein (Jantzen 2008, 96).

10 Armbruster 2000, 68; 71-72; Mödlinger 2007, 112; dies. 2011, 24-30; Jantzen 2008, 313-314.

11 Echte Lunker entstehen durch die Schrumpfung des Materials beim Abkühlungsprozess der Schmelze und enthalten keine Gase, sondern ein Vakuum (Bargel/Schulze 2008, 73).

12 Mödlinger 2007, 104; 113; dies. 2011, 33.

13 Dies. 2007, 113.

14 Armbruster 2000, 56; 125-126; Mödlinger 2007, 113; dies. 2011, 35-41.

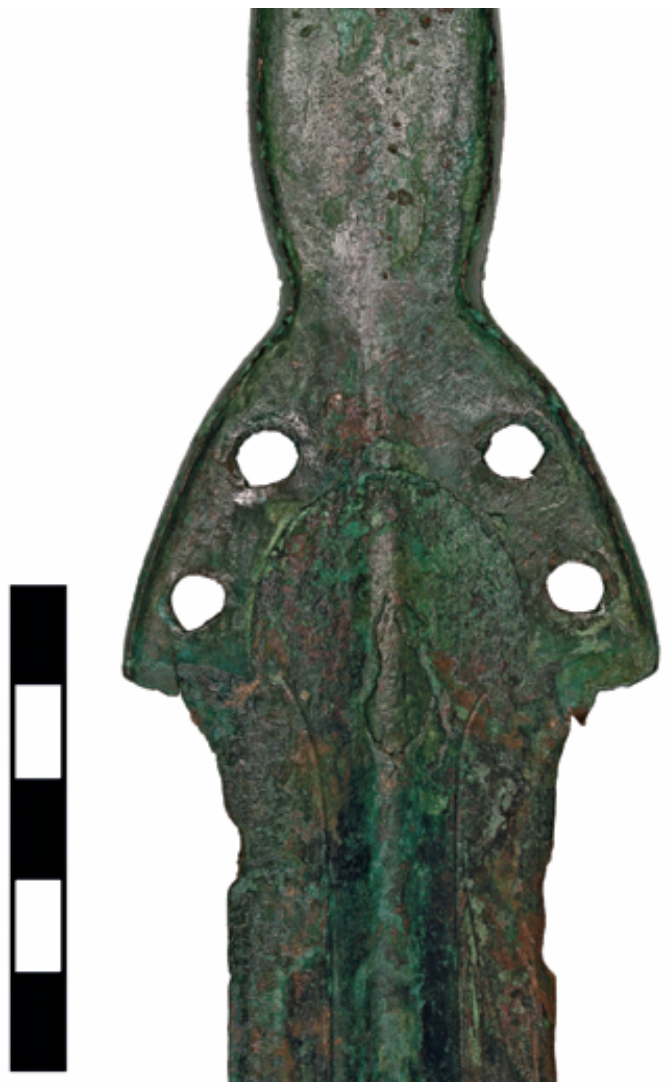

Abb. 1. Gussfehler auf der Klinge des Griffzungenschwertes aus der „Umgebung von Stade“ (Foto: S. Schwenzer)

Auch wenn im Fundmaterial keine metallographischen Untersuchungen vorgenommen werden konnten, ist eine effektive Nachbearbeitung von Schwertern in Niedersachsen für Kurzschwerter des Typs Dahlenburg (Per. III/IV) nachgewiesen $^{15}$. Zum Abschluss schliff und polierte man die Oberfläche mit geeigneten Materialien. Schleifen und Polieren dürften relativ zeitaufwendige Arbeiten gewesen sein, die neben der Nachbearbeitung gussfrischer Stücke auch dazu dienten Gebrauchspuren zu entfernen. Erhalten haben sich solche Bearbeitungsspuren in optimaler Weise an Stücken, die in Gewässern deponiert wurden und daher nur im geringen Maße Korrosion und Patinierung aufweisen. Patinierte und daher sicher antike Schleifspuren konnten im Rahmen des Projektes auf den Klingen von Lemförde (Nr. 14) und aus der „Umgebung von Bremen“ (Nr. 27) nachgewiesen werden (Abb. 3).

Mithilfe von Nieten wurden der Griff bzw. die Griffschalen aus organischem Material (beispielsweise Holz, Horn oder Bein) auf der Griffplatte und der Griffzunge befestigt. Teile der Griffschalen können gelegentlich auch aus Blei bestanden haben ${ }^{16}$. Darauf weist möglicherweise eine teilweise weißlich gefärbte Griffzunge beim Fundstück von Langen (Nr. 35) hin. Bei einem jungbronzezeitlichen Schwert aus Ra-

\footnotetext{
15 Kaufmann et al. 1996, 273-278.

16 Laux 2009, 99
} 

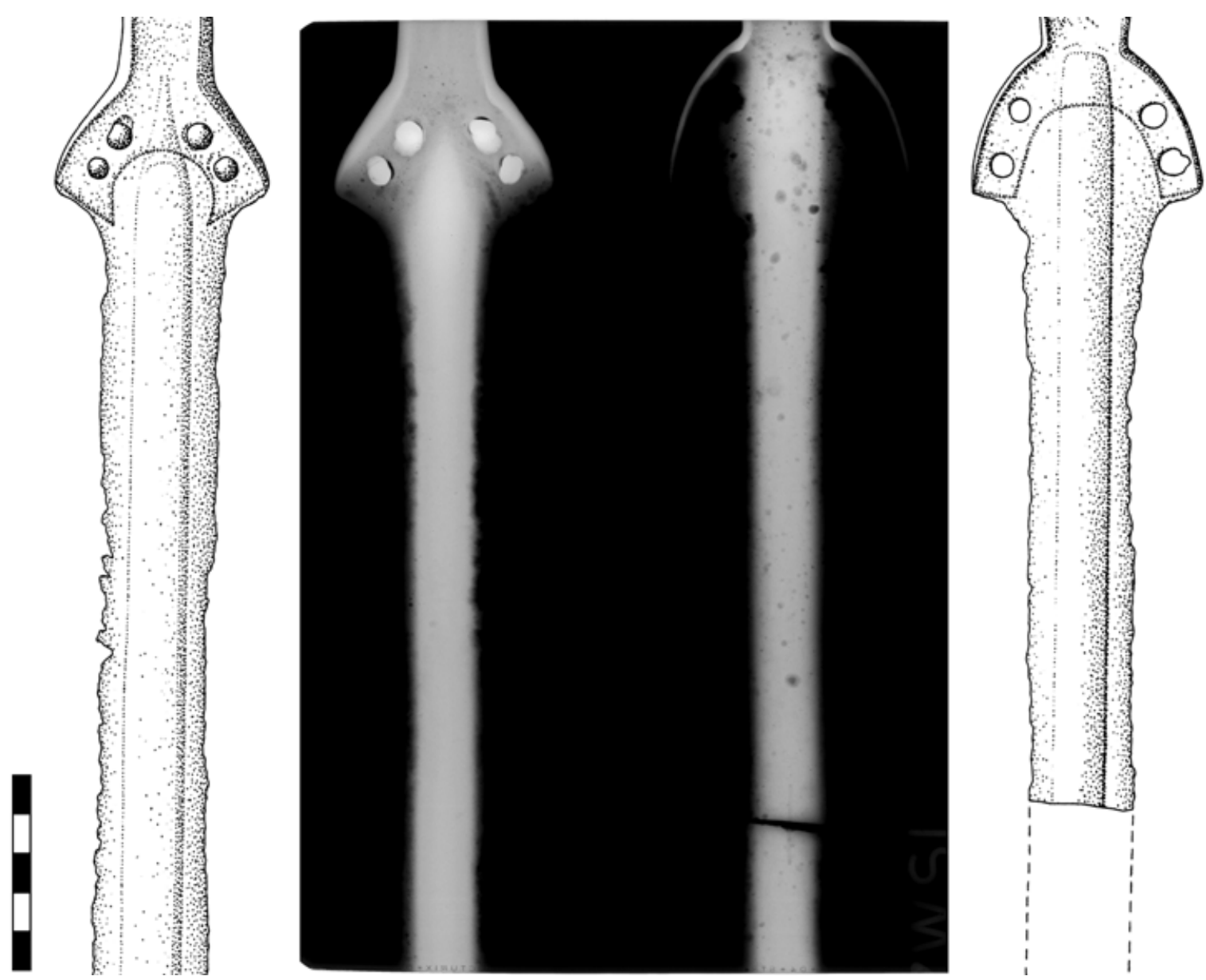

Abb. 2. Röntgenaufnahme der Griffzungenschwerter von Nindorf (links) und Harsefeld (rechts) (Zeichnungen: Laux 2009, Taf. 43,269 u. Taf. 39,246;

Röntgenaufnahme: Schloss Gottorf - Archäologisches Landesmuseum Schleswig)

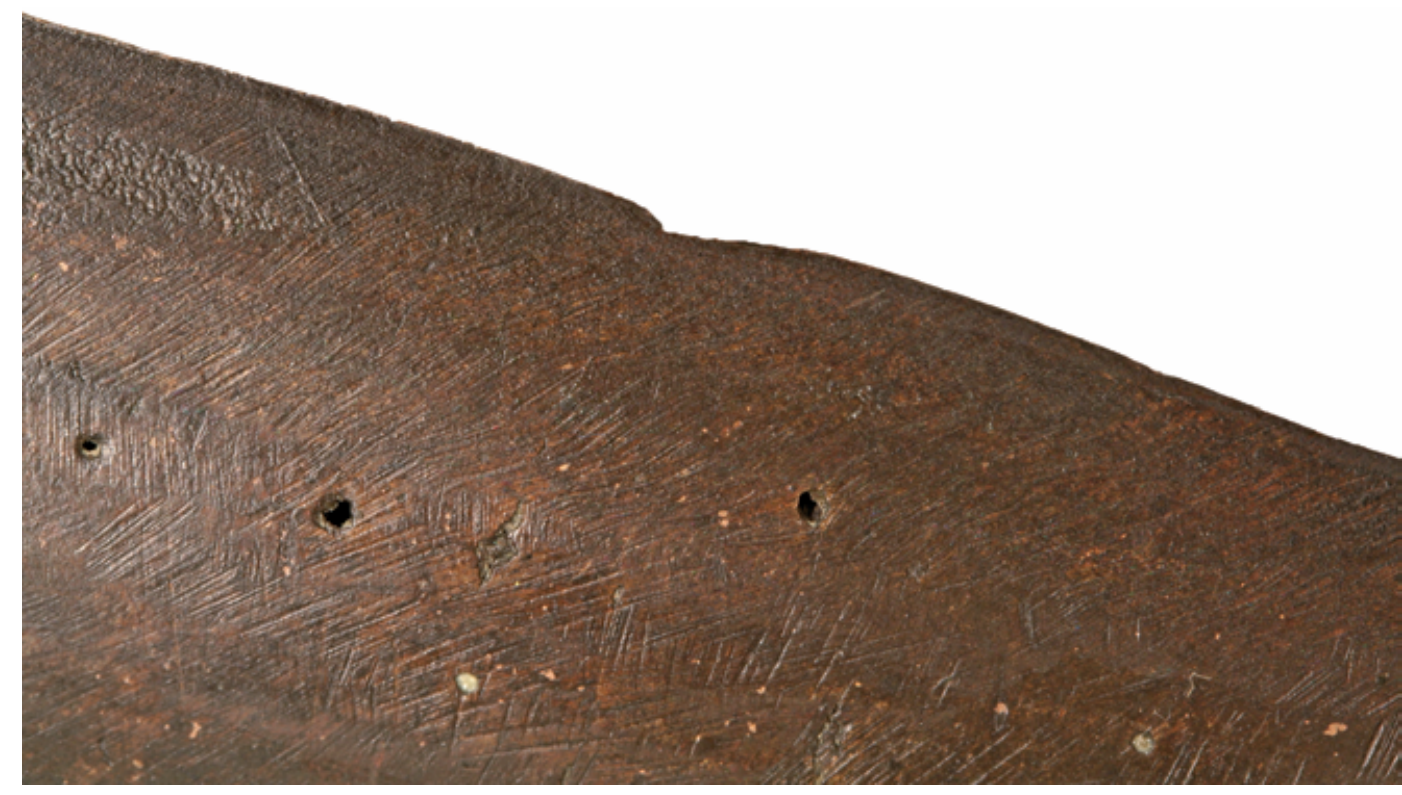

Abb. 3. Schleifspuren auf dem Schwert aus der „Umgebung von Bremen“ (ohne M; Foto: S. Schwenzer) 


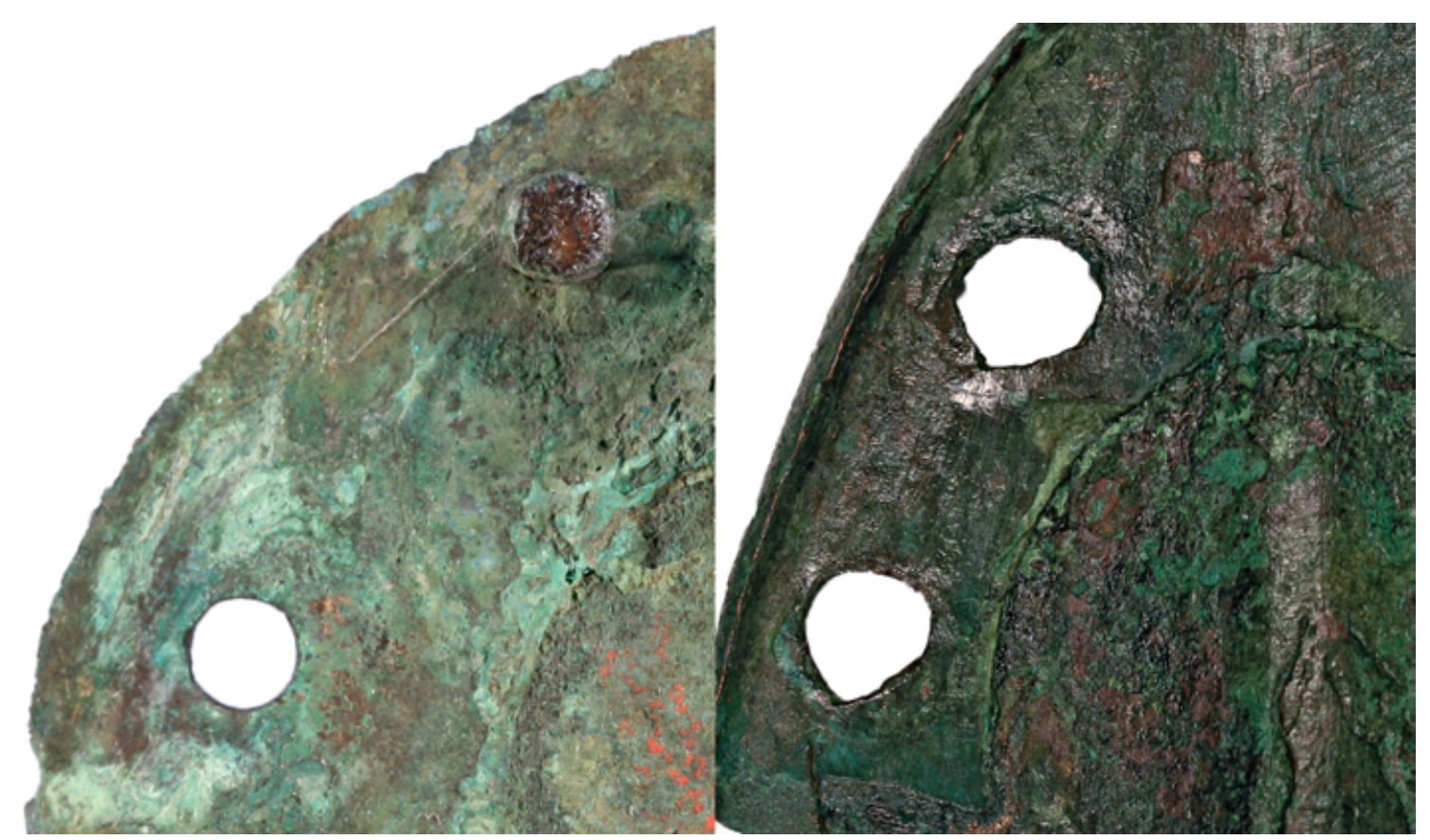

Abb. 4. Beispiele für ein vermutlich mitgegossenes Nietloch (links) und ein geschlagenes Nietloch (rechts) (ohne M; Fotos: S. Schwenzer)

depohl, Lkr. Parchim, Mecklenburg-Vorpommern, konnte kürzlich ein mehrschichtiger Aufbau aus dünnem Leder über dem Metall, einer dünnen Polsterung mit pflanzlichem Material, den hölzernen Griffschalen und einer Lederumwicklung nachgewiesen werden ${ }^{17}$.

Bei Griffplattenschwertern kommen Ringniete ${ }^{18}$ (Typen Sögel und Wohlde) und Pflockniete (Typen Baven und Harburg), bei Griffzungenschwertern nur Pflockniete vor ${ }^{19}$. Deutlich zu erkennen ist bei allen Schwertern, dass die inneren Niete länger sind als die äußeren; das organische Heft hatte offenbar einen gewölbten Querschnitt. Anhand unterschiedlich beschaffener Patina ist bei vielen Schwertern der Heftausschnitt rekonstruierbar, der bei den Griffplattenschwertern bogen-, halbkreis- oder dreiviertelkreisförmig und bei den Griffzungenschwertern meistens halbrund war.

Die Nietlöcher können theoretisch mitgegossen, gebohrt oder geschlagen sein. Hinweise auf mitgegossene Nietlöcher können regelmäßig geformte Löcher und Querschnitte sowie fehlende Aufwulstungen am Rand sein (Abb. 4). Denkbar ist nicht nur das Mitgießen von vollständigen Nietlöchern, sondern auch eine Anlage des Nietloches als

17 Schmidt 2009, 30.

18 Hier soll ebenso wie bei Laux (2009) der Begriff, ,Ringniet‘ für einen Niet mit relativ kegelförmigem oder pilzförmigem Kopf verwendet werden. Dies geschieht unabhängig davon, ob er einen massiven oder hohlen Querschnitt aufweist (anders als Schwenzer 2004a, 4), da diese Unterteilung im Fundmaterial schwierig durchzuführen ist. Alle Fundstücke, bei denen eine Unterscheidung möglich war, weisen jedoch einen massiven Querschnitt auf.

19 Laux 2009, 21; 28; 38-42; 98-127. eingetieftes „Senkloch“ beim Guss und eine nachträgliche Durchlochung 20 .

Eine Bohrung ist durch eine regelmäßige Form, charakteristische Bohrwülste mit Rissen darin und Bohrrillen im Loch zu erkennen ${ }^{21}$. H. Born nimmt den Gebrauch von Eisenspitzen $a^{22}$, während $\mathrm{H}$. Wüstemann eine Weiterentwicklung der neolithischen Steinbohrtechnik, die mit entsprechenden Schleifmitteln arbeitete, vermutet ${ }^{23}$. An dem Dolch von Dierkshausen, Lkr. Harburg, Niedersachsen, sind drei geschliffene Bohrungen, die jeweils von beiden Seiten durchgeführt wurden, nachzuweisen. Drei mitgegossene „Senklöcher" wurden dafür hier nicht durchlocht ${ }^{24}$.

Bei einer Herstellung der Löcher durch Guss oder Bohrung kommt es in jedem Fall in diesem ohnedies gefährdeten Bereich zu einem Materialverlust, der sich nachteilig auf die Widerstandskraft gegen Risse auswirkt.

Das Schlagen von Nietlöchern kann auf zwei Arten erfolgen (Abb. 4). Einerseits ist die Lochung mittels eines Runddornes in heißem oder kaltem Zustand denkbar. Dabei entstehen jeweils charakteristische Aufwulstungen am Rand der Löcher, die natürlich auch nachträglich abgearbeitet

20 Born 1989, 129 Abb. 3. Ein Beispiel hierfür findet sich bei zwei Gussformfragmenten für jungbronzezeitliche Schwerter von der „Hünenburg“ bei Watenstedt, Lkr. Helmstedt: Heske et al. 2010, 178 Anm. 14.

21 Born 1989, 129 Abb. 1.

$22 \mathrm{Zu}$ frühen Eisenwerkzeugen siehe auch Kapitel „Verzierung“.

${ }_{23}$ Drescher 1978b; Born 1989, 126; Armbruster 2000, 108; Wüstemann 2004, 214 Anm. 8.

24 Drescher 1956/58, 28 Taf. 5. 

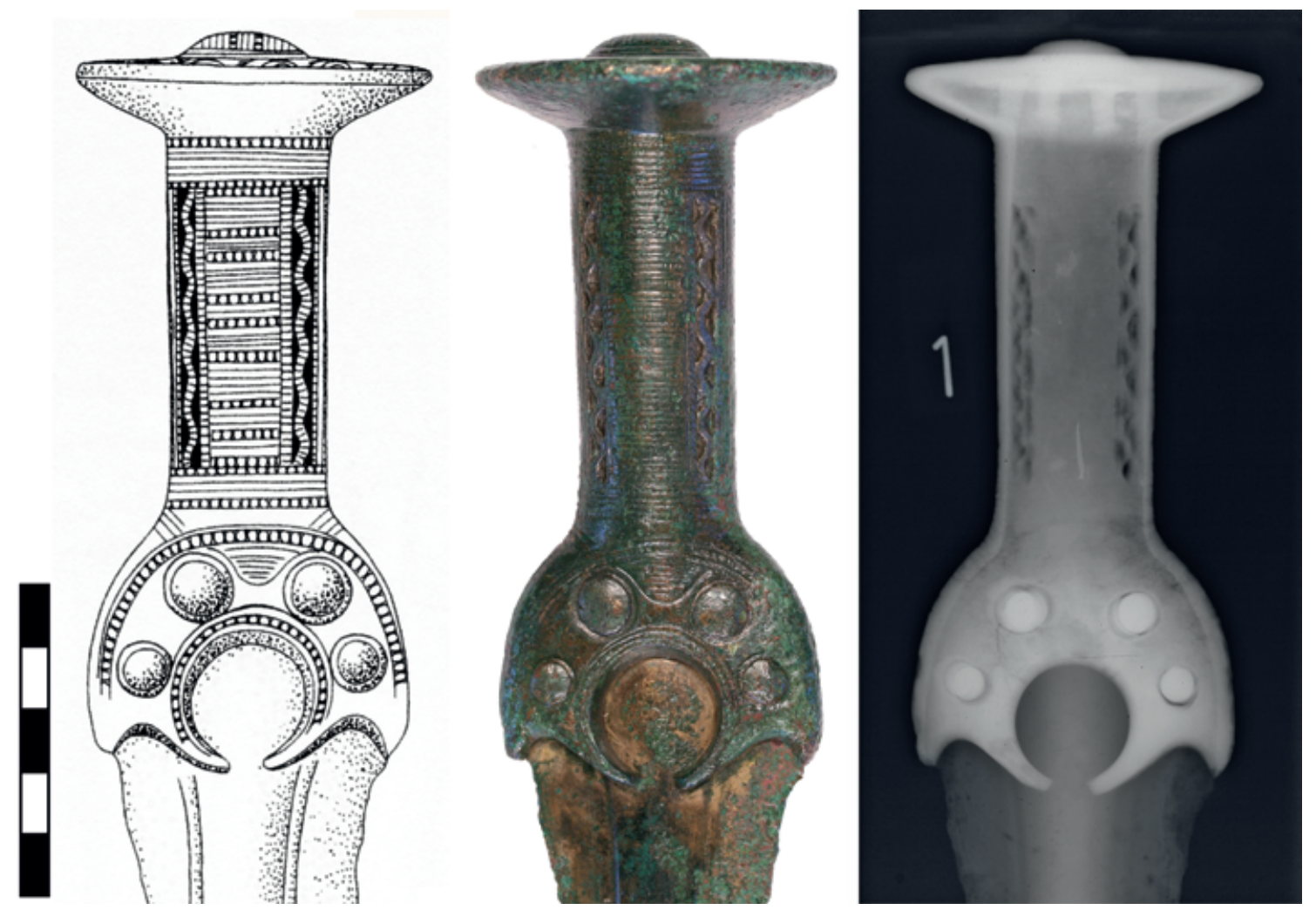

Abb. 5. Zeichnung, Foto und Röntgenaufnahme des nordischen Vollgriffschwertes von Wiepenkathen (Zeichnung: Laux 2009, Taf. 29,182; Foto: S. Schwenzer; Röntgenaufnahme: Römisch-Germanisches Zentralmuseum Mainz)

worden sein können ${ }^{25}$. Auch kleine Risse und Ausbrüche an den Rändern, die sich auf die Stabilität nachteilig auswirken, deuten darauf hin ${ }^{26}$. Am Griffzungenschwert von Toppenstedt, Lkr. Harburg, Niedersachsen, konnte Drescher andererseits nachvollziehen, dass an der betreffenden Stelle ein Ring vorgepunzt und die entstandene Scheibe dann mit einem Dorn heraus gestanzt wurde. Mit dieser Methode ist nach Drescher eine Durchlochung von bis zu etwa $2 \mathrm{~mm}$ starker Bronze mühelos möglich, darüber hinaus sei es umständlich oder unmöglich ${ }^{27}$. Manche der untersuchten Griffzungen scheinen mit einer Stärke von bis zu 3,5 mm für diese Technik deutlich zu dick zu sein.

In jedem Fall benötigte der Handwerker viel Übung und Erfahrung, um Nietlöcher möglichst ohne die kleinen Risse, die bei Belastung rasch weiter ausreißen können, zu schlagen.

An den niedersächsischen Griffplatten- und Griffzungenschwertern sind - trotz Korrosion, die derartige Beobachtungen erschwert - Hinweise auf mitgegossene, gebohrte sowie geschlagene Nietlöcher festzustellen. Gerade die frühen Griffplattenschwerter weisen in der Mehrzahl sehr regelmäßige Löcher auf, die wohl mitgegossen oder gebohrt

25 Armbruster 2000, 59; 111; Mödlinger 2007, 113; dies. 2011, $41-42$.

26 Wüstemann 2004, 125 Anm. 54.

27 Drescher 1956/58, 23-25. wurden, während die unregelmäßige Form der Griffzungenschwerter mit häufigen Ausbrüchen am Rand eher auf geschlagene Löcher schließen lässt.

Es deutet sich also eine chronologische Tendenz von früheren mitgegossenen oder gebohrten hin zu späteren geschlagenen Nietlöchern an.

\section{Vollgriffschwerter}

\section{Untersuchungen am Griff - Nordische Vollgriffschwerter}

Der Griff nordischer Vollgriffschwerter und -dolche wurde im sog. „nordischen Feinguss“ über einem Tonkern hergestellt. Diese Bezeichnung bezieht sich auf den sehr dünnwandigen und materialsparenden Guss der Griffwandung und des Knaufs. Der innere Hohlraum entspricht dem äußeren Griffprofil somit weitgehend (Abb. 5)28. Für die Periode II existieren unterschiedliche Meinungen zur Gusstechnik der Vollgriffschwerter: Einmal wird von zweiteiligen Gussformen bzw. Klappformen gesprochen ${ }^{29}$, andererseits vom Guss im Wachsauschmelzverfahren ${ }^{30}$. Da ins-

28 Ders. 1961, 57; Wüstemann 2004, 213-214 Anm. 6.

29 Drescher 1961, 57; Oldeberg 1976, 79; Wüstemann 2004, 213 Anm. 6.

30 Driehaus 1968, 362-363; Drescher 1978a, 61-62; Wüstemann $1999,138$. 

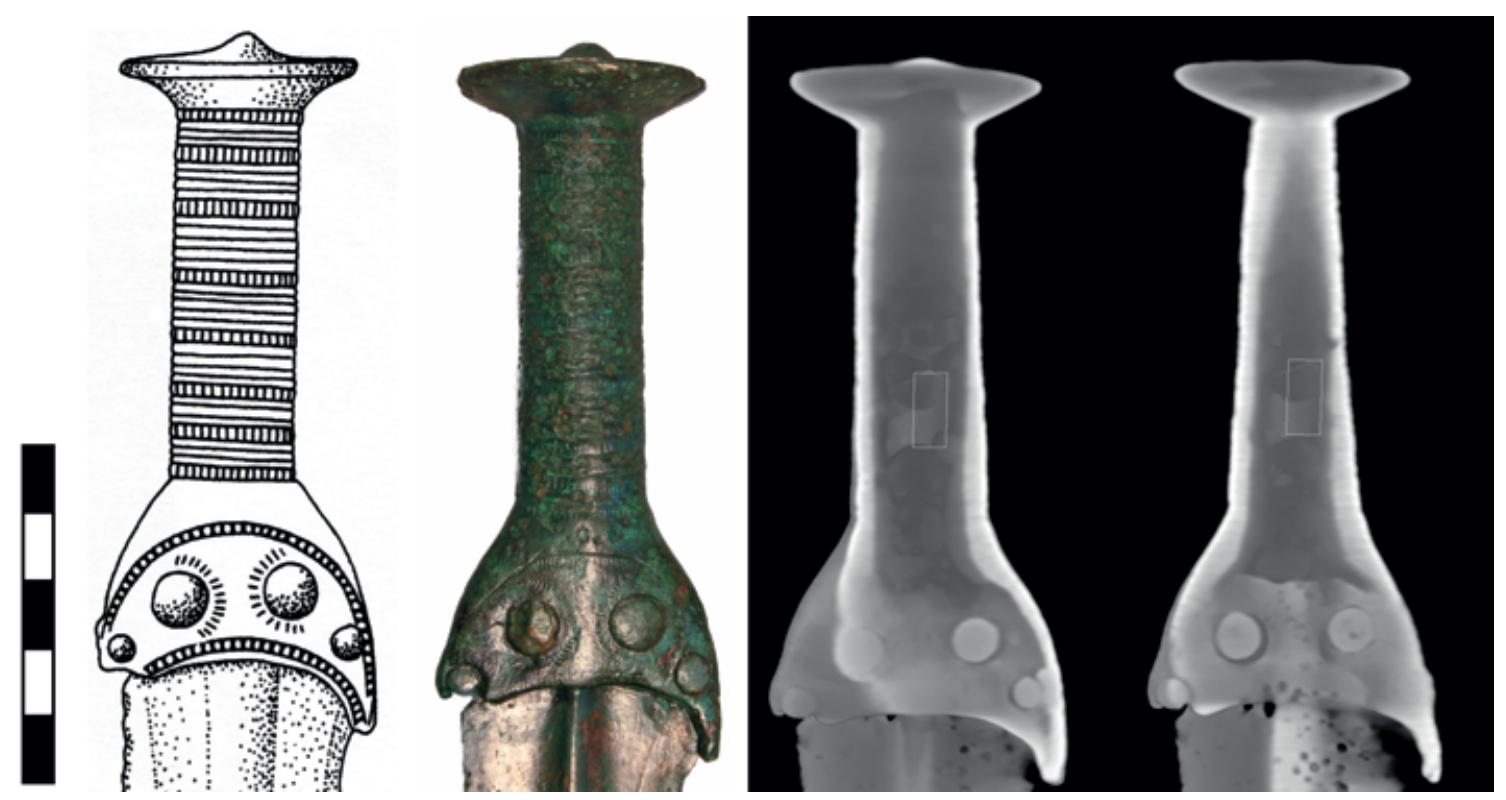

Abb. 6. Zeichnung, Foto und zwei computertomographische Aufnahmen des nordischen Vollgriffschwertes von Apensen ([Nr. 41]; Zeichnung: Laux 2009, Taf. 30,190; Foto: S. Schwenzer; Computertomographie: Volkswagen AG, Wolfsburg)

gesamt nur eine einzige bronzezeitliche Schwertgriffgussform in Europa bekannt ist (für Griffe des Typs Mörigen, Fundort Erlingshofen, Lkr. Eichstätt, Bayern) und die Griffe gerade der nordischen Vollgriffschwerter sehr individuell geformt sind, ist vom Wachsausschmelzverfahren als Norm auszugehen. Es wird vermutet, dass der Einguss zweiarmig von den Heftenden her erfolgte, da die Luft aus dem Kern so direkt in den Formmantel entweichen konnte. Vor allem wirkt der Knauf zu dünnwandig und instabil für die Montage eines Gusstrichters und das Entfernen eines Gusszapfens. Möglicherweise hing der Kern an den Metalloder Tonstiften, die die großen und mitgegossenen Nietlöcher am Heft aussparen sollten, und benötigte daher keine weiteren Kernhalter ${ }^{31}$.

Diese komplexe Gusstechnik ist an vielen Fundstücken in Niedersachsen, die bemerkenswert qualitätsvoll hergestellt sind und nur wenige Lunker oder Gasblasen aufweisen, deutlich erkennbar. Bei vielen Schwertern - unter anderem den computertomographisch untersuchten Fundstücken (Nr. 41, 42, 48, 53) - wurde der Gusskern zumindest teilweise im Griff belassen, was eine Arbeitsersparnis bedeutete und zur Stabilität beitrug, aber auch den Schwerpunkt der Waffe in Richtung Griff verändert. Bei den CT-Untersuchungen konnte außerdem durch die raue Gussoberfläche im Griffinnenraum Ton als Kernmaterial nachgewiesen werden $^{32}$.

Nicht vollkommen gelungen ist der Guss bei einem Schwert von Apensen (Nr. 41), da große Teile des eigentlichen Knaufinnenraumes mit Bronze gefüllt sind (Abb. 6).

\footnotetext{
31 Driehaus 1968, 361-362; Drescher 1978a, 61; Wüstemann 2004, 214 Anm. 7.

32 Vgl. auch Mödlinger 2008.
}

Es ist unwahrscheinlich, dass es sich hier um einen Nachguss handelt, da nicht einmal in der computertomographischen Untersuchung Einschnitte im Metall erkennbar sind $^{33}$. Im unteren Bereich des Griffes ist der Tonkern an vielen Stellen gestört, wohl weil man versucht hatte, ihn zu entfernen.

Ein zweites Schwert von Apensen (Nr. 42) offenbart einen Überfangguss am Knauf, mit dem vermutlich ein misslungener Guss nachgebessert wurde (Abb. 7). Eventuell befand sich der Angusspunkt bei diesem Schwert entgegen der allgemeinen Annahme am Knauf und die Entfernung eines Gusszapfens führte zur Beschädigung der Knaufplatte, die anschließend repariert werden musste. Große Teile der Kreisaugenornamentik sind durch Korrosion nicht mehr erkennbar, andere Bereiche aber noch sehr gut. Möglicherweise ist das unterschiedliche Korrosionsverhalten ebenfalls auf den Nachguss mit anderer Materialzusammensetzung zurückzuführen.

Das Schwert aus der „Umgebung von Stade“ (Nr. 51) zeigt eine bislang einmalige Gusstechnik (Abb. 8). Die Form des Griffinnenraumes folgt nicht den Konturen des Griffes, sondern verjüngt sich zum Heft hin und ist nach unten möglicherweise geschlossen. Zumindest besteht zwischen den Innenräumen von Griff und Heft eine im Röntgenbefund dünne Materialverstärkung. Ein in diesem Bereich genauerer Befund könnte gegebenenfalls durch eine dreidimensionale Computertomographie erzielt werden, was jedoch leider im Rahmen des Projektes nicht mehr möglich war.

33 Nachgüsse verschmelzen nicht mit dem vorher vorhandenen Material, sondern sind nur mechanisch verbunden: Drescher 1958,9 . 

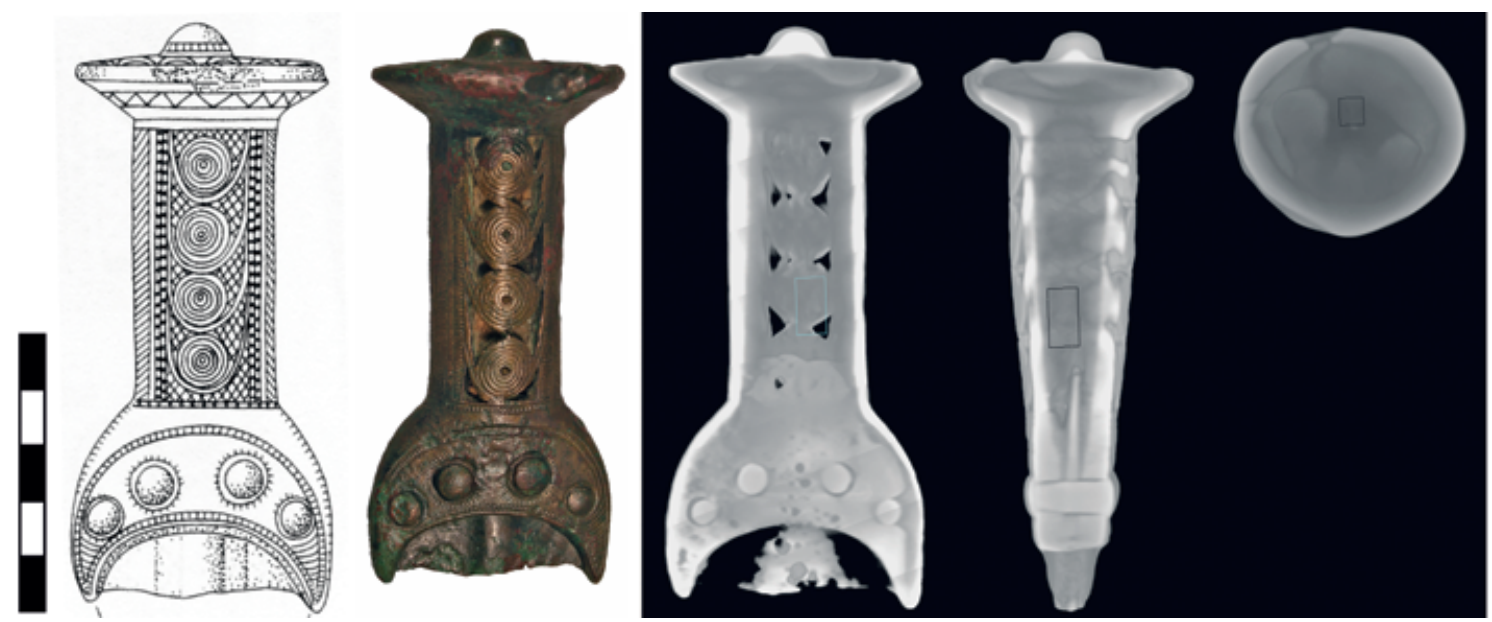

Abb. 7. Zeichnung, Foto und drei computertomographische Aufnahmen (frontal, seitlich, Knauf von oben) des nordischen Vollgriffschwertes von Apensen ([Nr. 42]; Zeichnung: Laux 2009, Taf. 29,185; Foto: S. Schwenzer; Computertomographie: Volkswagen AG, Wolfsburg)
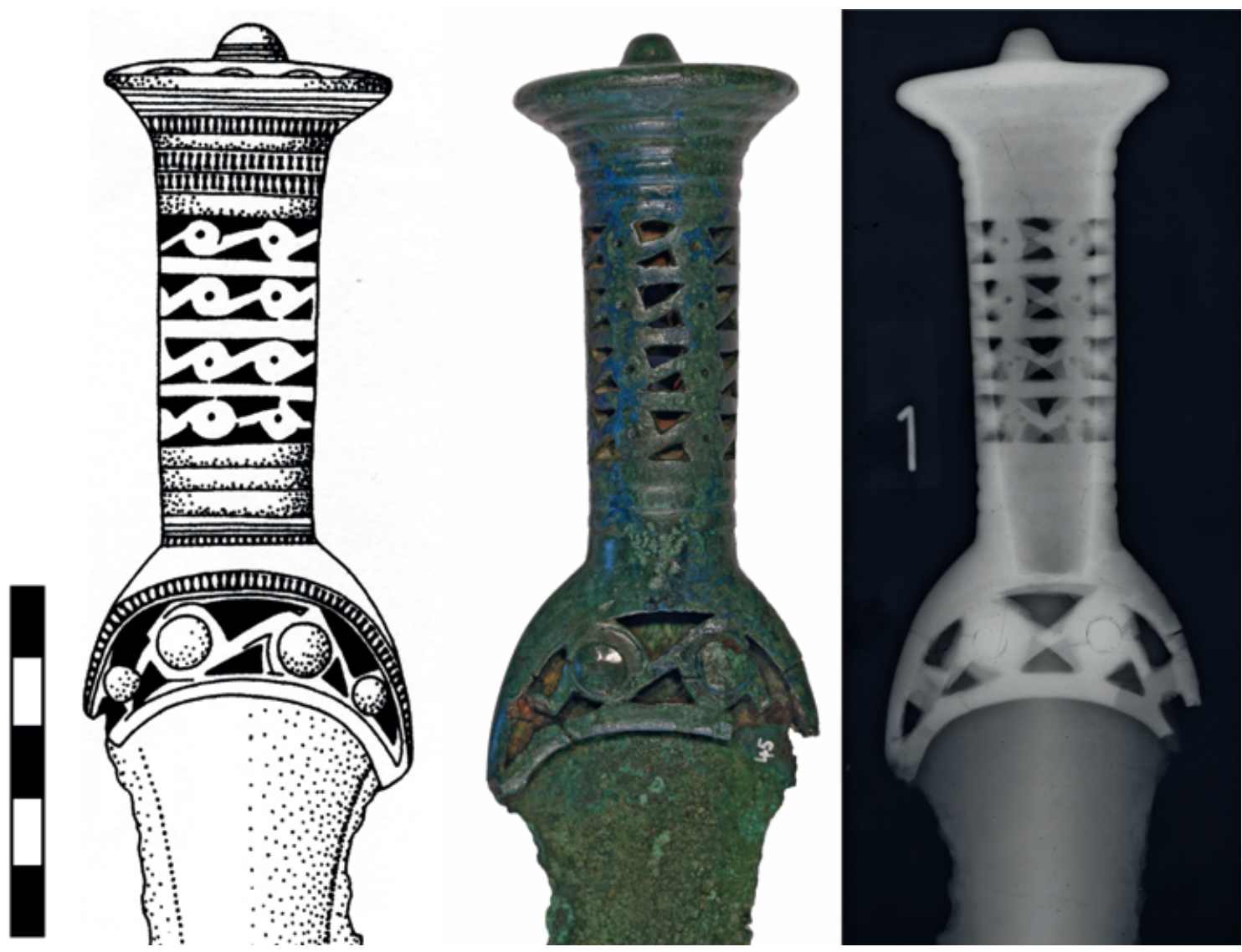

Abb. 8. Zeichnung, Foto und Röntgenaufnahme des nordischen Vollgriffschwertes aus der „Umgebung von Stade“ ([Nr. 51]; Zeichnung: Laux 2009, Taf. 30,188; Foto: S. Schwenzer; Röntgenaufnahme: Römisch-Germanisches Zentralmuseum Mainz) 

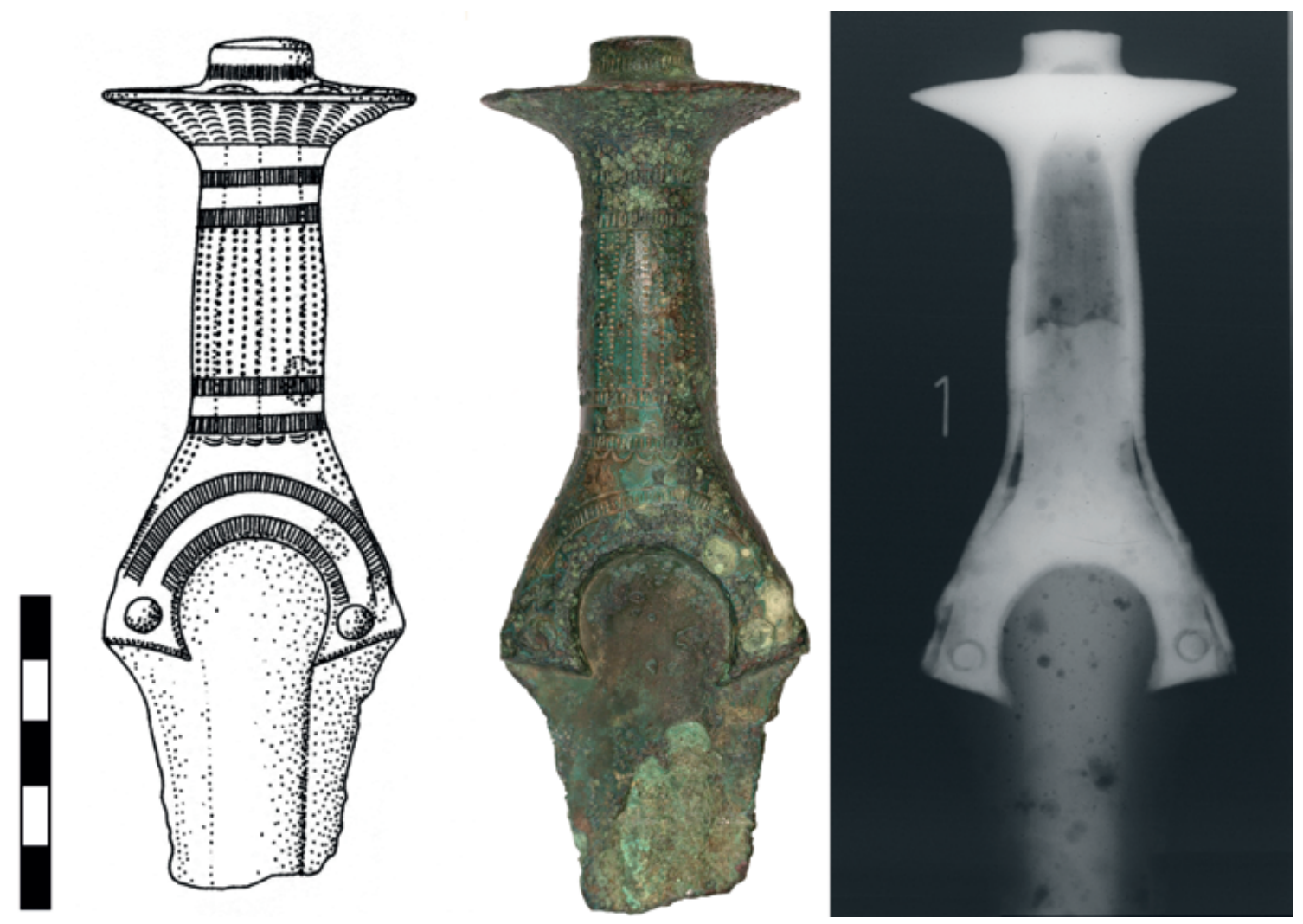

Abb. 9. Zeichnung, Foto und Röntgenaufnahme des Achtkantschwertes von Schwinge (Zeichnung: Laux 2009, Taf. 25,170; Foto: S. Schwenzer; Röntgenaufnahme: Römisch-Germanisches Zentralmuseum Mainz)

Auch in Periode III wurden die Griffe der Vollgriffschwerter nordischen Typs nach einhelliger Meinung im Wachsausschmelzverfahren gegossen. Dies trifft auch für die im Rahmen des Projektes aufgenommenen Schwerter von Deutsch Evern (Nr. 43) und Meckelfeld (Nr. 49) zu. Im Falle des von Drescher untersuchten Schwertes von Meckelfeld wurden Knauf und Griff wohl absichtlich einzeln gegossen und der schmale Schlitz dazwischen wohl mit organischem Material kaschiert. Weiterhin ist an diesem Fundstück zu erkennen, dass der Gusskern vier Rillen aufweist. Dies erleichterte den Guss, da das Material die dünnwandigen Bereiche des Heftes schneller ausfüllen konnte. Eventuell lag der Einguss im oberen Rand des Griffteils und erfolgte mit vier Armen. Auch für Schwerter der Periode III ist ansonsten aber ein zweiarmiger Einguss an den Heftenden denkbar, so dass der Kern in die Form gehängt werden konnte. Im Knauf befindet sich unterhalb der Platte ein Steg, der parallel zu den Seiten in der Mitte verläuft und auf irgendeine unbekannte Art und Weise mit der Angel verbunden ist ${ }^{34}$.

34 Ders. 1961, 60-61.

\section{Untersuchungen am Griff - Achtkantschwerter}

Nach Ausweis der bisher bekannt gewordenen und untersuchten Funde sind alle Achtkantschwerter, egal ob sie im südlichen Mitteleuropa oder in Nordeuropa gefunden wurden, in vergleichbarer Weise hergestellt worden (Abb. 9). Man hatte sie wahrscheinlich, ebenso wie andere Vollgriffschwerter, im Wachsauschmelzverfahren gegossen. Ein Kern diente dazu, den Griffinnenraum beim Guss auszusparen. Die Verwendung von Klappformen ist nicht gänzlich auszuschließen. Allerdings diente der oben erwähnte, europaweit bislang einzige Fund einer solchen Form aus Erlingshofen, Lkr. Eichstätt, Bayern, dem Guss von Schwertern des Typs Mörigen, ist also jünger zu datieren. Wüstemann vermutet außerdem den Guss in verlorener Sandform ${ }^{35}$. Beim Guss stand die Form wohl aufrecht, wobei sich der Einguss vermutlich im Knaufbereich befand. Anders als bei den nordischen Vollgriffschwertern ist der Griff der Achtkantschwerter sehr dickwandig und der Knauf massiv gegossen. Im Knauf befindet sich dennoch meistens ein unregelmäßiger und nach außen offener Hohlraum. Das kleine Loch wurde oft durch einen Bronzestift oder Hämmern verschlossen und ist häufig nicht mehr

35 von Quillfeldt 1995, 75; 214 Nr. 281; Wüstemann 2004, 122-123 Anm. 45; Mödlinger 2007, 112; dies. 2011, 31-32. 

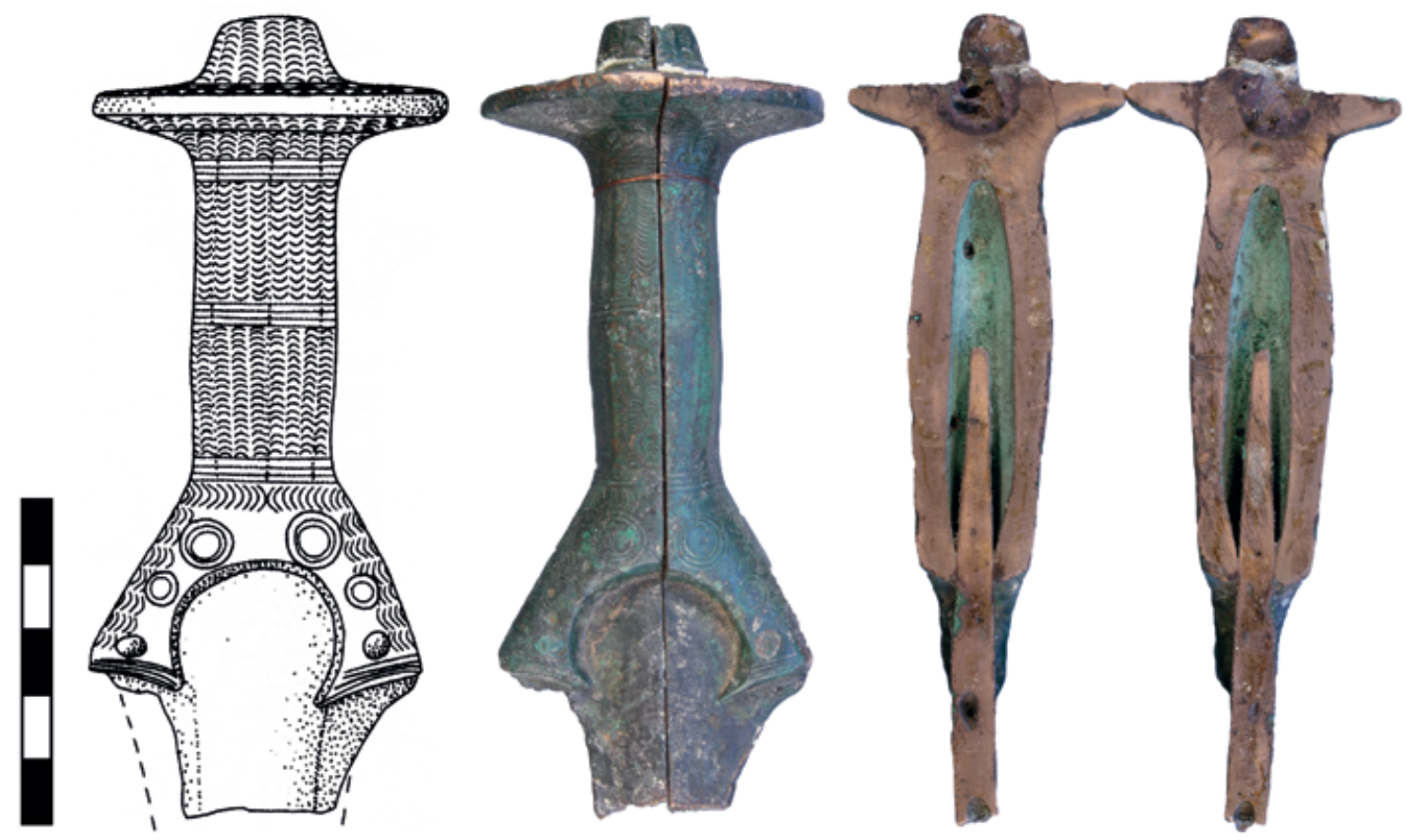

Abb. 10. Zeichnung und zwei Fotos des Achtkantschwertes von Bremervörde (frontal, Anschnitt)

(Zeichnung: Laux 2009, Taf. 26,172; Fotos: S. Schwenzer)

sichtbar ${ }^{36}$. Das Profil des Griffinnenraumes entspricht nicht dem Außenprofil, sondern verjüngt sich zum Knauf hin. Nach dem Guss wurde der Tonkern offenbar in der Regel vollständig entfernt, um Platz für die Griffzunge zu schaffen ${ }^{37}$. Die Nietlöcher im Heftbogen wurden mitgegossen.

Alle im Rahmen dieses Projektes angefertigten bzw. ausgewerteten Röntgenbilder der niedersächsischen Achtkantschwerter zeigen den typisch dickwandigen Guss und zum Teil Gasblasen in den Griffen. Das Schwert von Bremervörde (Nr. 55) wurde nicht geröntgt, aber rezent in Längsrichtung durchgeschnitten (nicht durch die Projektausführenden!) (Abb. 10). Sehr gut zu erkennen ist zum einen aufgrund der rauen Innenwand die Verwendung eines Tonkerns, zum anderen im Knaufbereich eine schwammige Struktur. Hierbei handelt es sich wohl um Mikrolunker, die beim Schwund des Metalls während der Abkühlung der Schmelze entstanden sind. Ähnliches lässt sich im Röntgenbild auch beim Fundstück von Kuhla (Nr. 57) im Bereich des Knaufknopfes erkennen. Außerdem zeigen sich auch kleinere Fehler. So ist beim Schwert aus der „Umgebung von Stade“ (Nr. 63) der Tonkern offenbar beim Guss verrutscht, so dass der Griffinnenraum schief im Griff liegt (Abb. 11).

\footnotetext{
36 Hundt 1965, 45.

37 Soweit erkennbar wurde bei allen Schwertern in Süddeutschland, Ostdeutschland und Niedersachsen der Tonkern entfernt, vgl. auch von Quillfeldt 1995, 76 und Wüstemann 2004, 122-123.
}

Lange Zeit wurde diskutiert, auf welche Art der unregelmäßige Hohlraum in vielen Knäufen entstanden ist (Abb. 9; $12 ; 20)^{38}$. Eine Theorie besagt, dass er von entweichenden Gasen verursacht wurde; es bleibt aber fraglich, warum es bei manchen Schwertern - auch in Niedersachsen - keinen solchen Hohlraum gibt ${ }^{39}$. Driehaus sieht als mögliche Erklärung einzig das (ihm unerklärliche) Umstürzen der Gussform direkt nach dem Einguss, wobei noch flüssiges Material aus dem Knauf wieder heraus fließ $\mathrm{e}^{40}$. Mödlinger erklärt den Hohlraum mit einer Fixierung des Kerns ${ }^{41}$; in diesem Fall sollte der Hohlraum jedoch einen regelmäßigeren und zielgerichteten Verlauf aufweisen ${ }^{42}$. Eine plausible Erklärung ist, dass es sich um Innenlunker handelt, die durch den Schwund des Materials beim Abkühlungsprozess entstehen, wenn die Schmelze bereits komplett von erstarrtem Metall eingeschlossen ist. Je nach Größe und Ausdehnung dieses Innenlunkers konnte so bei der Entfernung des Gusszapfens eine kleine Öffnung nach außen entstehen ${ }^{43}$. Zur Vermeidung solcher Hohlräume bemühen Gießer sich in der Regel um eine relativ gleichmäßige Materialstärke im gesamten Gussstück, die eine gleichmäßige Abkühlung des Metalls gewährleistet. Bei Achtkantschwer-

\footnotetext{
38 Vgl. u.a. Hundt 1965, 45; von Quillfeldt 1995, 75-76; Wüstemann 1999, 135

39 Ders. 2004, 123.

40 Driehaus 1968, 356-357.

41 Mödlinger 2007, 112; dies. 2011, 32.

42 Driehaus 1968, 356.

43 Auch wenn Driehaus $(1968,357)$ keine Notwendigkeit dafür erkennt, spricht nichts gegen das Vorhandensein von Gusszapfen bei der Herstellung von Achtkantschwertern.
} 

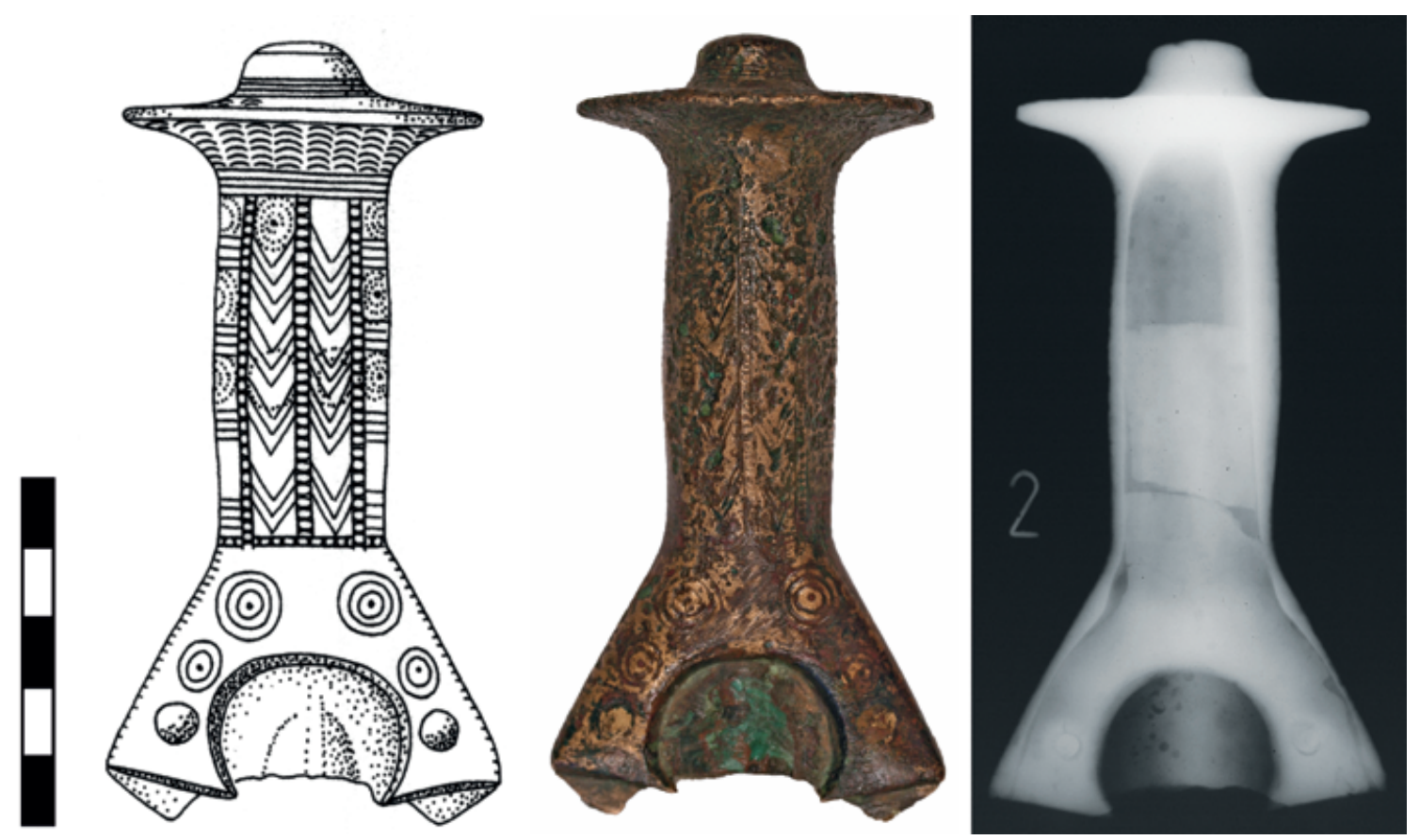

Abb. 11. Zeichnung, Foto und Röntgenaufnahme des Achtkantschwertes aus der „Umgebung von Stade“

(Zeichnung: Laux 2009, Taf. 28,179; Foto: S. Schwenzer;

Röntgenaufnahme: Römisch-Germanisches Zentralmuseum Mainz)

tern ist der Knaufbereich jedoch viel massiver als die übrigen Bereiche, weshalb eine hohe Wahrscheinlichkeit besteht, dass sich Innenlunker bilden. Derartige Lunker können sich nicht nur als größere Hohlräume ausprägen, sondern ebenso in Form zahlreicher Mikrolunker auftreten (siehe die Schwerter von Bremervörde [Nr. 55, Abb. 10] und Kuhla [Nr. 57]) $)^{44}$.

Interessant sind die Schwerter von Meckelstedt (Nr. 59, Abb. 12) und Wedel (Nr. 64, Abb. 13), die Abweichungen von der üblichen Gusstechnik aufweisen. Das Fundstück von Meckelstedt, das von F. Laux typologisch als Vorform der Achtkantschwerter angesprochen wird ${ }^{45}$, zeigt einen recht dünnwandig gegossenen Griff. Der Innenraum verjüngt sich nur unwesentlich zum Knauf, der einen Innenlunker aufweist. Diese Details deuten auch von technischer Seite mit ihrer Ähnlichkeit zur Gussweise des Typs Spatzenhausen eine frühe Zeitstellung an. Das Schwert von Wedel, von Laux typologisch als Zwischenform von Typ Spatzenhausen und Achtkantschwertern bezeichnet ${ }^{46}$, hat einen dünnwandig gegossenen Griff mit konischem Hohlraum bis in den Knaufbereich. Diese Befunde stimmen mit den von Driehaus und Hundt für den Typ Spatzenhausen herausgestellten Merkmalen überein ${ }^{47}$. Ob auch beim Schwert von Wedel der Gusskern - wie beim Typ Spatzenhausen üblich -

\footnotetext{
${ }_{44} \mathrm{Zu}$ Innenlunkern und ihrer Entstehung vgl. Bargel/Schulze 2008, 73-74.

45 Laux 2009, 71 Nr. 168.

46 Ebd. 70-71 Nr. 167

47 Driehaus 1961, 25-26; Hundt 1965, 43-44.
}

im Griff belassen wurde, ist nicht erkennbar. Am Knauf weist der Griff einen deutlich erkennbaren Überfangguss auf (Abb. 14), der in einem Loch am ehemals flachovalen Knaufknopf verankert ist. Der Nachguss ist vielleicht im Auftrag des nordischen Besitzers angefertigt worden, um den Knauf nach Art der nordischen Knäufe zu vergrößern und anschließend mit Spiralen verzieren zu können ${ }^{48}$.

Offenbar stimmt bei diesen beiden Fundstücken die typologische Ansprache mit den herstellungstechnischen Beobachtungen überein, so dass hier Vorgänger der Achtkantschwerter zu fassen sind. Hundt deutet das Schwert von Wedel hingegen als parallele Entwicklung zu den Achtkantschwertern, die zwar die Schäftungstechnik übernahm, aber eine alte Gusstechnik und Form beibehielt. Das Interesse an südmitteleuropäischen Schwertern beschränkte sich im Norden jedenfalls nicht auf echte Achtkantschwerter, sondern schloss verwandte und möglicherweise etwas frühere Formen mit ein. Ein Schwert, das nach Meinung von Hundt werkstattgleich mit dem von Wedel ist, fand sich in Matrei in Osttirol, Bezirk Lienz, Tirol, Österreich ${ }^{49}$.
48 Hundt $1979,185$. 

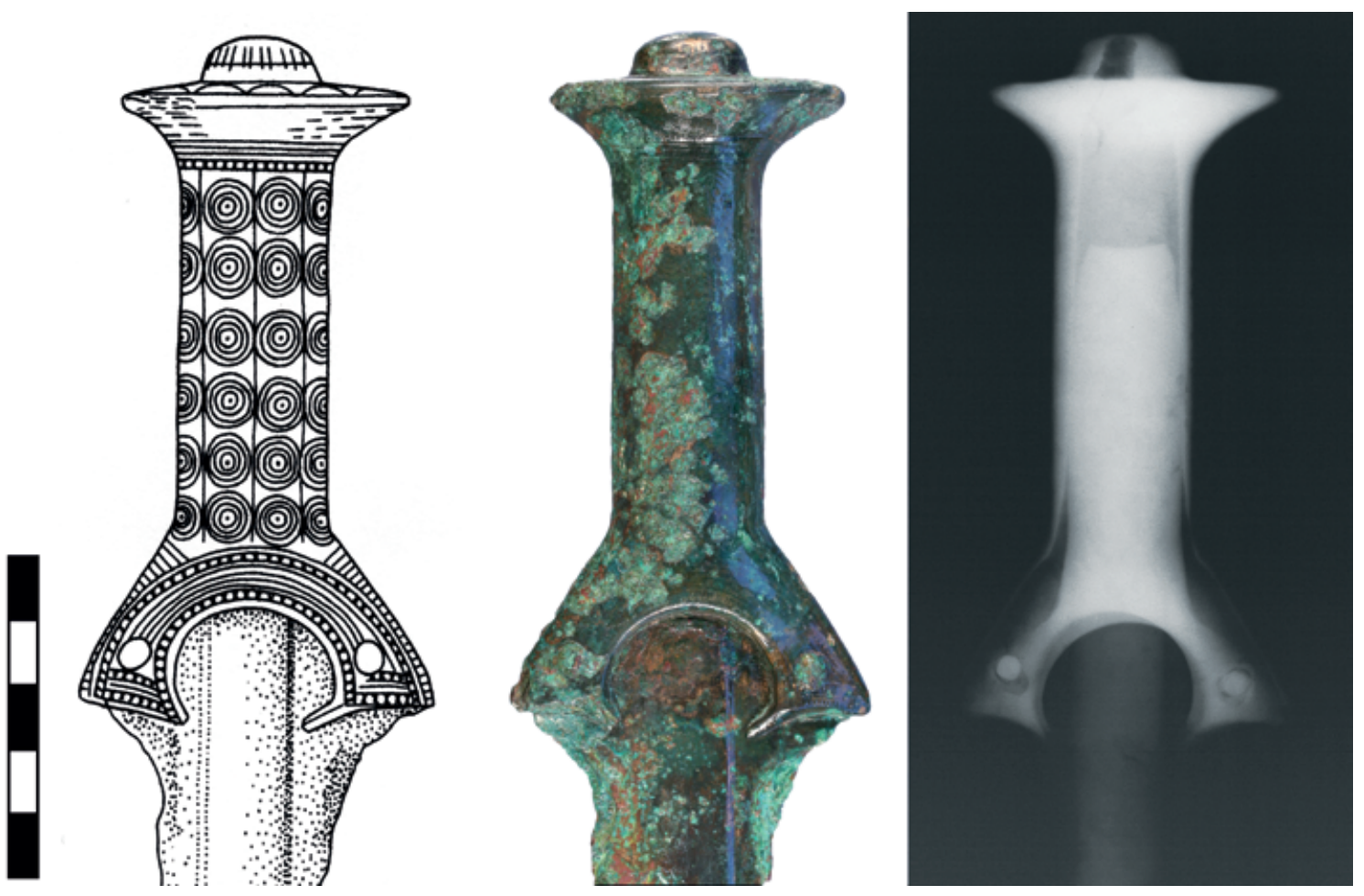

Abb. 12. Zeichnung, Foto und Röntgenaufnahme des Achtkantschwertes von Meckelstedt (Zeichnung: Laux 2009, Taf. 25,168; Foto: S. Schwenzer;

Röntgenaufnahme: Römisch-Germanisches Zentralmuseum Mainz)
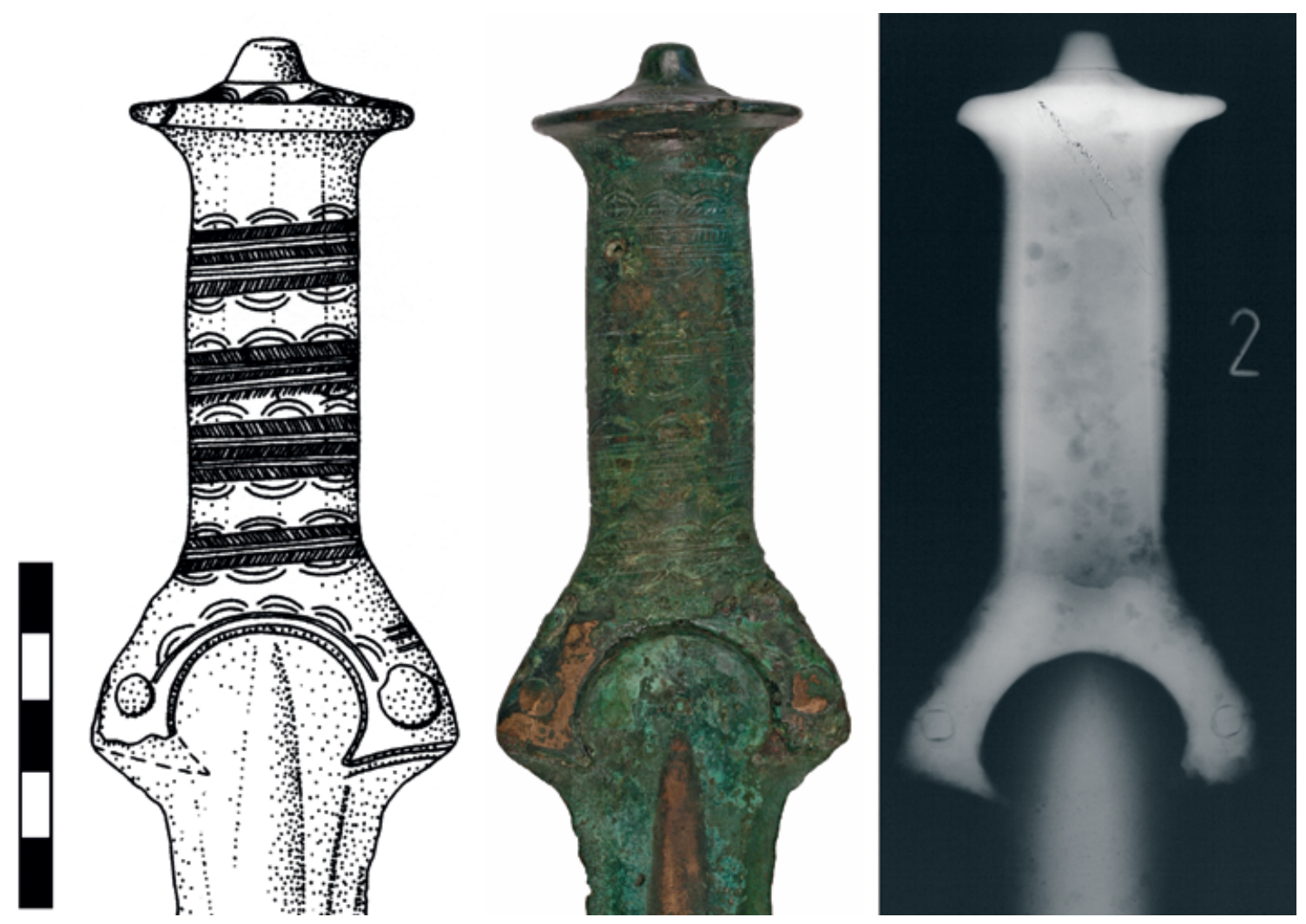

Abb. 13. Zeichnung, Foto und Röntgenaufnahme des Achtkantschwertes von Wedel (Zeichnung: Laux 2009, Taf. 25,167; Foto: S. Schwenzer; Röntgenaufnahme: Römisch-Germanisches Zentralmuseum Mainz) 


\section{Untersuchungen am Griff - einteilig gegossene Fundstücke}

Weiterhin sind zwei Vollgriffdolche von Hambostel (Nr. 66) und aus der „Umgebung von Stade“ (Nr. 69) und ein kurzes Vollgriffschwert von Ruschwedel (Nr. 68, Abb. 15) untersucht worden, die mithilfe von Scheinnieten am Heft den Anschein einer mit dem Griff verbundenen Klinge erwecken. Tatsächlich sind sie jedoch massiv und einteilig gegossen.

\section{Untersuchungen an der Klinge}

Die Klingen der Vollgriffschwerter wurden in zweiteiligen Gussformen angefertigt und später nachbearbeitet ${ }^{50}$. Der Angusspunkt kann über die Verteilung der Gasblasen in der Klinge lokalisiert werden ${ }^{51}$, bei den niedersächsischen Vollgriffschwertern sind jedoch keine Konzentrationen erkennbar, weil meist nur die Griffpartien radiographisch untersucht sind. Es gibt offenbar sowohl Klingen mit vielen als auch solche mit sehr wenigen Blasen.

Die Klingen der nordischen Vollgriffschwerter von Loxstedt (Nr. 48, Abb. 19) und Westerwanna (Nr. 53) sind offenbar nicht erst durch Nachschärfungen stark gekürzt, sondern gezielt in der vorliegenden Länge hergestellt worden. Dafür sprechen die sich gleichmäßig verschmälernden Mittelrippen.

Auf welche Art die Nietlöcher in den Klingen angebracht wurden, ist fraglich. Wüstemann zufolge wurden sie erst nach dem Guss passend zum jeweiligen Griff angebracht, weil der Schwund durch Trocknung und Brand der keramischen Gussformen sowie durch Abkühlung des Metalls es seiner Ansicht nach erschwerte, die Nietlöcher von Griff und Klinge deckungsgleich zu gießen ${ }^{52}$. Einige Jahre zuvor ging Wüstemann noch wie Driehaus, A. Oldeberg und Drescher davon aus, dass die Nietlöcher bereits beim Guss ausgespart wurden ${ }^{53}$. Für nachträglich angebrachte Nietlöcher kommen Schlagen und Bohren in Frage, wie bei den Griffplatten- und Griffzungenschwertern bereits dargestellt wurde (s.o.). Im Allgemeinen sind die Nietlöcher an den Klingen der nordischen Vollgriffschwerter größer und regelmäßiger geformt als die der Achtkantschwerter mit ihren Unregelmäßigkeiten und Ausbrüchen. Beim Schwert von Meckelstedt (Nr. 59, Abb. 12) wurde ein Nietloch offenbar sogar an falscher Stelle angebracht und zur Korrektur des Fehlers ein zweites Loch leicht versetzt ausgeführt. Dies belegt auf jeden Fall die Praxis der nachträglichen Lochung.

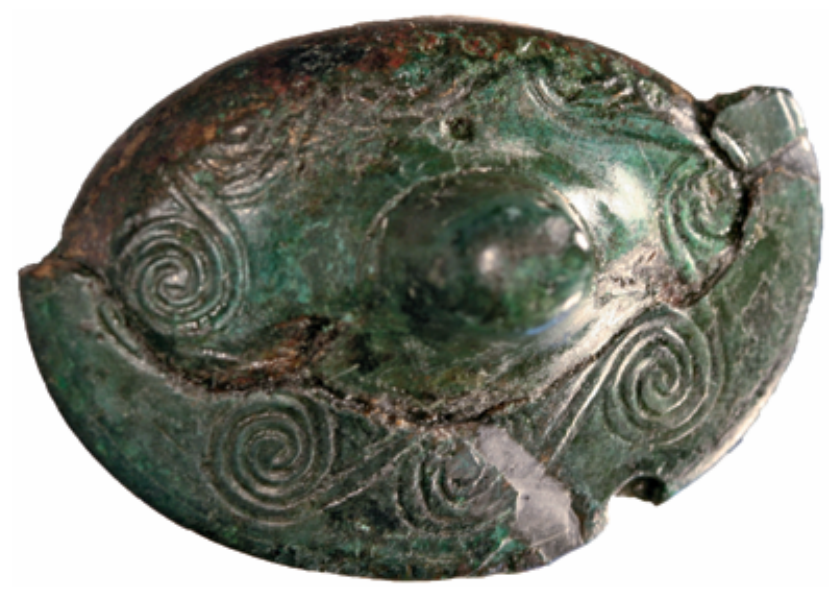

Abb. 14. Knauf des Achtkantschwertes von Wedel (ohne M; Foto: S. Schwenzer)

Untersuchungen an der Schäftung - Nordische Vollgriffschwerter

Die Funktionalität von Vollgriffschwertern wird maßgeblich durch die Technik bestimmt, mit der die Klinge mit dem Griff verbunden ist.

Griff und Klinge der nordischen Vollgriffschwerter sind durch Niete und nicht durch Überfangguss, wie früher gelegentlich angenommen wurde, miteinander verbunden ${ }^{54}$. In Periode II sind verschiedene Schäftungsarten zu unterscheiden. Zum einen können die Niete am Heft oder Knauf befestigt sein; außerdem gibt es unterschiedlich gestaltete Klingenenden mit Griffplatten, Griffzungen oder Griffangeln. Weiterhin interessant sind die Genauigkeit der Einpassung der Klinge in das Heft sowie die Anzahl der Niete bzw. Scheinniete, die meist zwischen vier und sechs liegt ${ }^{55}$. Insgesamt sind die Nietlöcher und Niete der nordischen Vollgriffschwerter sehr regelmäßig geformt und qualitativ besser als bei den Achtkantschwertern ${ }^{56}$.

Die Schwerter aus der „Umgebung von Stade“ (Nr. 51, Abb. 8) und von Wiepenkathen (Nr. 54, Abb. 5) besitzen am Abschluss der Klingen gerundete Griffplatten, die in ihrer Größe und Form dem Heftmund entsprechen. Die Klingen der Fundstücke von Apensen (Nr. 41, Abb. 6; Nr. 42; Abb. 7) und Harsefeld (Nr. 47) hingegen könnten nach den Röntgenaufnahmen ursprünglich eine Griffzunge besessen haben, die aber zu einem unbekannten Zeitpunkt abbrach bzw. abgebrochen wurde. Bei allen drei Fundstücken stimmen die Form von Griffplatte und Heft nicht exakt überein. Bei den Schwertern von Apensen finden sich sogar völlig ausgebrochene Nietlöcher (Abb. 6 und Abb. 7). Offenbar wurde die Klinge bei jenen Stücken nicht für diesen Griff konzipiert; ob hier möglicherweise der Griff oder die Klinge sekundär verwendet wurde, ist nicht zu sagen.

\footnotetext{
50 Drescher 1961, 60; Mödlinger 2007, 112-113; dies. 2011, 30.

51 Dies. 2007, 104; dies. 2011, 33; 76.

52 Wüstemann 2004, 214 Anm. 7.

53 Driehaus 1968, 362; Oldeberg 1976, 80; Drescher 1978, 62; Wüstemann 1999, 140.
}

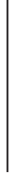

\footnotetext{
54 Drescher 1958, 75-76; Wüstemann 2004, 213-224; 229-233.

55 Drescher 1961, 57; Ottenjann 1969, 10.

56 Vgl. Wüstemann 2004, 215 Anm. 9.
} 

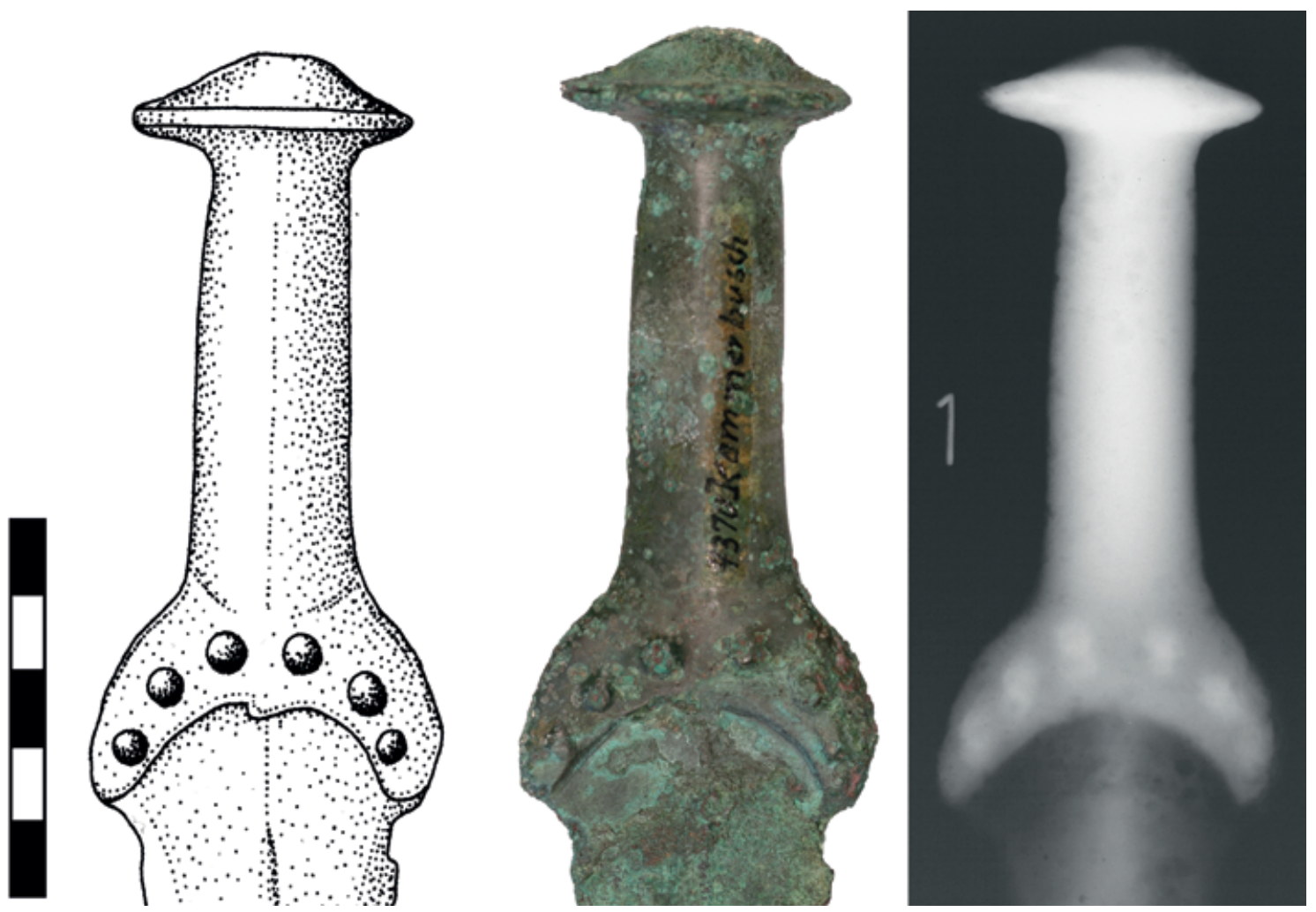

Abb. 15. Zeichnung, Foto und Röntgenaufnahme des einteilig gegossenen Vollgriffschwertes von Ruschwedel (Zeichnung: Laux 2009, Taf. 1,2; Fotos: S. Schwenzer;

Röntgenaufnahme: Römisch-Germanisches Zentralmuseum Mainz)

Das Schwert von Estorf (Nr. 44, Abb. 16) hat eine zum Heftmund passende Klinge. Die Griffzunge verläuft etwa bis zur Mitte des Griffteils und ist wie bei den Achtkantschwertern (s.u.) mit den Innenwänden der Griffsäule verkeilt. Die Verwendung dieser Verkeilschäftung bei nordischen Vollgriffschwertern ist auch von Fundstücken aus Schleswig-Holstein und Mecklenburg-Vorpommern bekannt $^{57}$.

Die Schwerter aus der „Umgebung von Stade“ (Nr. 52, Abb. 17) und Westerwanna (Nr. 53, Abb. 18) haben hingegen eine Griffangelklinge, deren Angel durch die Griffsäule bis zum Knauf führt. Beim erstgenannten Fundstück passt sich das Klingenende nicht exakt dem Heft an und die Griffangel ist einmal, möglicherweise durch die Stauchung beim Vernieten, gebrochen. Ähnlich wie bei dem „westlichen“ Vollgriffschwert von Meißenheim, BadenWürttemberg, erfassen die beiden kleineren äußeren Niete die Klinge nur zum Teil und die größeren inneren sind nur Scheinniete $^{58}$. Etwas besser gelang die Einpassung der Klinge an das Heft beim Fundstück von Westerwanna. Die

\footnotetext{
57 Schleswig-Holstein: Puls (Driehaus 1968, 358 Abb. 27; 361); Mecklenburg-Vorpommern: Stralsund (Wüstemann 2004, 218 Nr. 553), Lehsen (ebd. 220 Nr. 557), Silmenitz (ebd. 224 Nr. 563), „Umgebung von Brüsewitz“ (ebd. 216 Nr. 548).

58 Hundt 1962, 36; 55.
}

recht breite Griffangel ist aber im unteren Bereich an einer Stelle gebrochen und im oberen Bereich stark gestaucht. Sie war offenbar zu lang und wurde gewaltsam in den Griff eingepasst. Ob die Risse rund um die Nietlöcher alt oder rezent, eventuell im Zusammenhang mit dem Bruch der Klinge, entstanden sind, muss offen bleiben. Ein im unteren Bereich der Griffsäule bei äußerlicher Betrachtung wie ein Niet wirkender Teil der Verzierung kann durch die Röntgenanalyse als Scheinniet identifiziert werden. Die Klinge war im Heft mit vier Nieten befestigt, von denen nur drei erhalten sind.

Ungewöhnlich nicht nur in Hinblick auf die Befestigung der Klinge ist die Konstruktionsweise des Schwertes von Loxstedt (Nr. 48, Abb. 19). Es fehlt das Heft, welches wohl aus organischem Material gefertigt war, da der saubere untere Abschluss der Griffsäule gegen einen Bruch spricht. Parallelen zu dieser Konstruktionsweise sind uns nicht bekannt. Die Einpassung der Klinge lässt sich daher und wegen des sehr fragmentarischen Zustandes der Griffplatte nicht mehr beurteilen.

Nordische Vollgriffschwerter der Periode III haben durchgängig eine Klinge mit Griffangel. Die durch die Griffsäule des Vollgriffes verlaufende Griffangel, wurde am Knauf durch ein Loch geführt und verhämmert. Dadurch mündet das Ende der Griffangel meist in einem kleinen Knaufknopf. Am Heft befinden sich ausschließlich Scheinoder Zierniete, die eine entsprechende Verbindung imitie- 

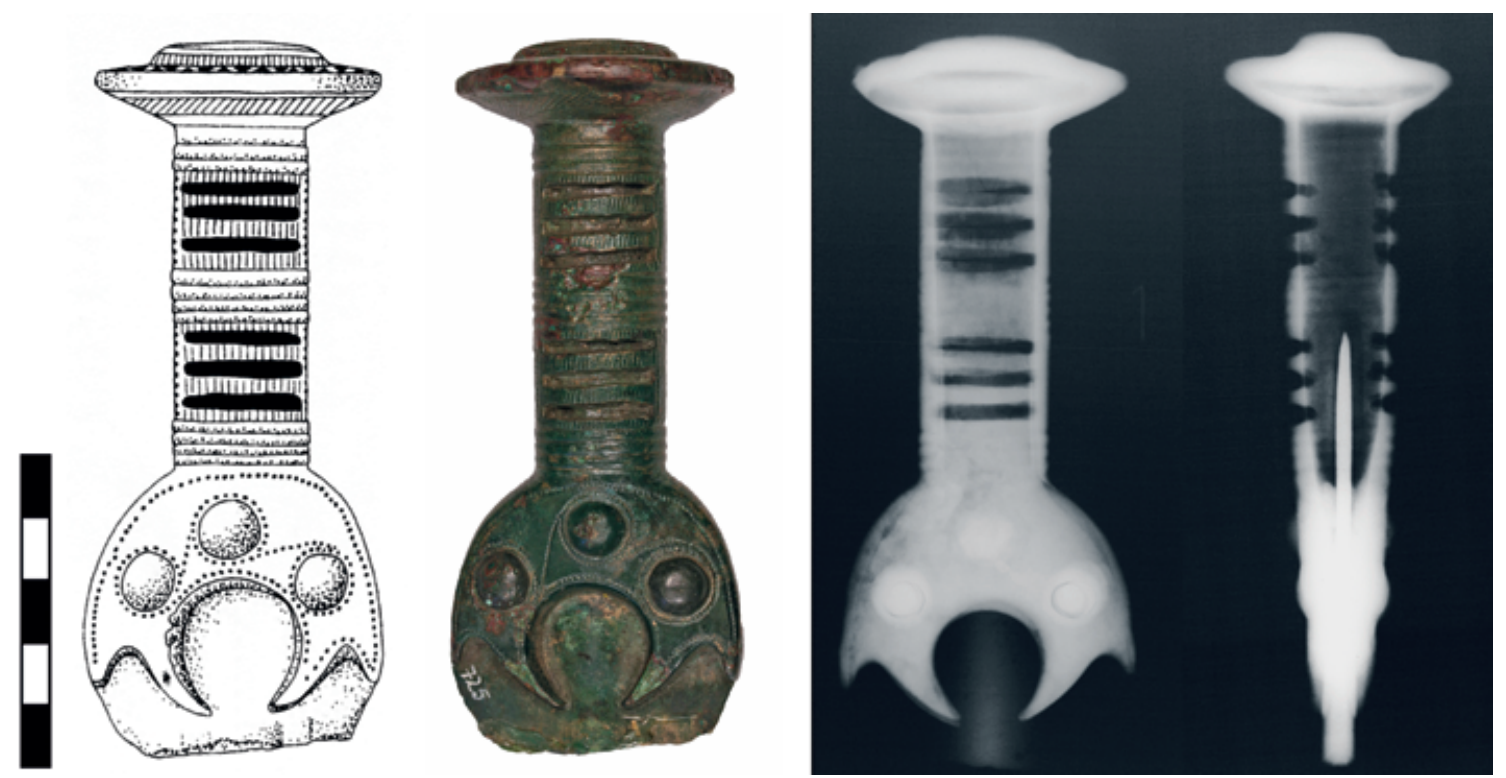

Abb. 16. Zeichnung, Foto und Röntgenaufnahmen (frontal, seitlich) des nordischen Vollgriffschwertes von Estorf (Zeichnung: Laux 2009, Taf. 29,183; Foto: S. Schwenzer;

Röntgenaufnahmen: Römisch-Germanisches Zentralmuseum Mainz)
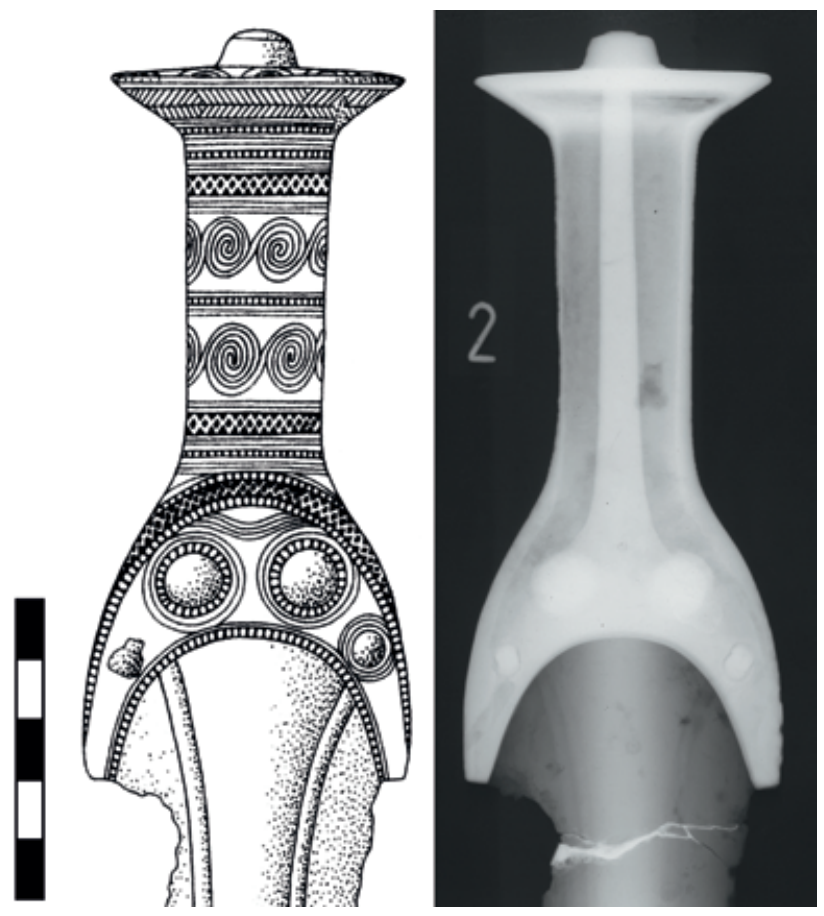

Abb. 17. Zeichnung und Röntgenaufnahme des nordischen Vollgriffschwertes aus der „Umgebung von Stade“ (Nr. 52) (Zeichnung: Laux 2009, Taf. 31,196; Röntgenaufnahme: Römisch-Germanisches Zentralmuseum Mainz) 

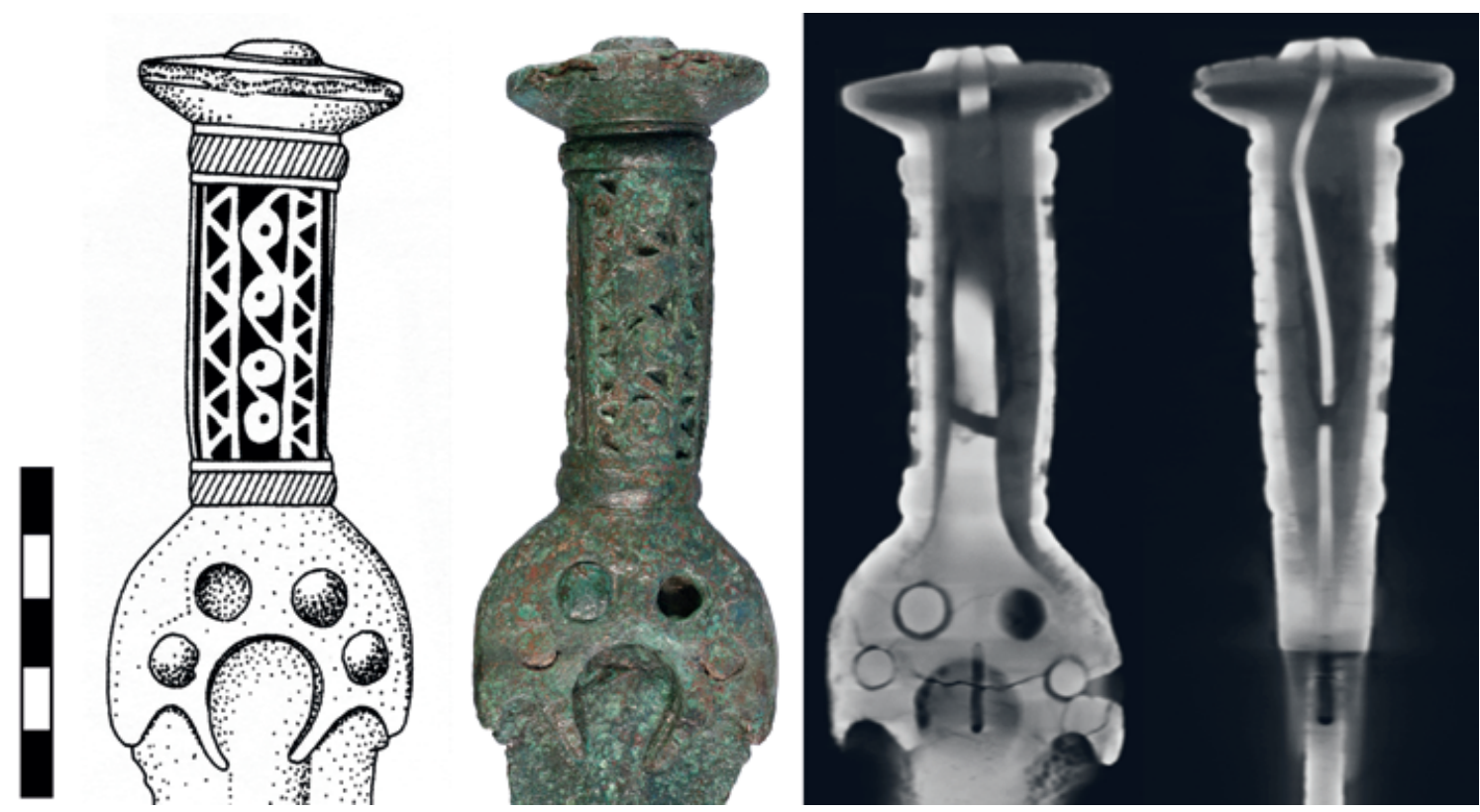

Abb. 18. Zeichnung, Foto und computertomographische Aufnahme des nordischen Vollgriffschwertes von Westerwanna (Zeichnung: Laux 2009, Taf. 30,186; Foto: S. Schwenzer; Computertomographie: Volkswagen AG, Wolfsburg)

ren $^{59}$. Beim Schwert von Deutsch Evern (Nr. 43) schließt die aufgesetzte Griffpartie eng an der Griffangelklinge ab. Vermutlich ist dies dadurch zu erklären, dass das Wachsmodell für die Gussform direkt an der Klinge angepasst wurde. Anders als bei einer Herstellung durch den (ebenfalls theoretisch denkbaren) Überfangguss blieben so beide Teile voneinander trennbar und der Heftabschluss konnte nachbearbeitet werden. Anschließend wurde der Heftmund entsprechend angeschmiedet. Ein weiterer Vorteil dieses Vorgehens ist, dass im Falle eines Fehlgusses kein weiterer Schaden an der bereits fertigen Klinge verursacht wurde ${ }^{60}$.

Für das Schwert von Meckelfeld (Nr. 49) vermutet Drescher, dass die Schäftung von zahlreichen kleinen Holzpflöcken zwischen dem sehr harten Tonkern und der Griffangel stabilisiert wird ${ }^{61}$.

Auffällig ist die hohe Individualität der Verbindungstechniken in Periode II, während in Periode III nur noch eine einzige Variante existiert. Ein breiter angelegter Vergleich der technischen Befunde mit $\mathrm{H}$. Ottenjanns anhand von Form und Ornament definierten Typen könnte helfen, die von ihm vermuteten Werkstätten bzw. Werkstattkreise entweder zu bestätigen oder ein neues Bild zu entwerfen ${ }^{62}$.

\section{Untersuchungen an der Schäftung - Achtkantschwerter}

Die Schäftungsweise der Achtkantschwerter ist deutlich weniger abwechslungsreich als die der zeitgleichen nordischen Vollgriffschwerter der Periode II.

59 Wüstemann 2004, 231-232.

60 Drescher 1958, 75-76; Wüstemann 2004, 232.

61 Drescher 1961, 61.

62 Vgl. Hundt 1962, 20; Ottenjann 1969.
Die als Vorgänger der Achtkantschwerter zu bewertenden Schwerter des Typs Spatzenhausen haben eine Klinge mit abgerundeter Griffplatte, die mit zwei Nieten nahe den Heftenden im Heft befestigt wurde. Während der Griff noch den größten Teil des Gusskerns enthält und daher stabil ist, stellt der Heftbereich, der ebenso dünnwandig gegossen ist und keinen Kern mehr aufweist, bei diesen Waffen einen kritischen Punkt dar ${ }^{63}$. Hebelkräfte konnten hier leicht zu einer Beschädigung der Verbindung führen. Dies war vermutlich der Grund, die Schäftungstechnik, wie bei den Achtkantschwertern zu beobachten ist zu verändern und zu verbessern.

Bei den eigentlichen Achtkantschwertern wurde die Schäftung auf eine neuartige Weise ausgeführt (Abb. 9). Auch hier finden sich am Heft nahe den Heftenden zwei Niete. Die Breite an dieser Stelle liegt in einem einheitlichen Bereich von $5,4 \mathrm{~cm}$ bis $5,8 \mathrm{~cm}$. Die Klinge endet jedoch nicht in einer halbrunden Heftplatte, sondern hat eine in den Griff hineinragende Heftzunge. Die Schultern der Klinge sind am Übergang zur Heftzunge meist einziehend geformt. Im Heftinnenraum und in der unteren Griffsäule hält die Griffzunge häufig keinen Kontakt zur Wandung des Griffs. Die Zunge berührt dann meistens etwa in der Mitte der Griffsäule deren innere Seiten und ist damit verkeilt, da der Griffinnenraum sich zum Knauf hin verjüngt. Selten ragt die Griffzunge dabei über die Mitte der Griffsäule hinaus. Das genaue Einpassen der Klinge an die Heftbreite sowie der Zunge an den Griffinnenraum geschah durch Ausschmieden. Nach der Vernietung wurde möglicherweise noch das Heft an die Klinge angeschmiedet ${ }^{64}$.

63 Hundt 1965, 44.

${ }^{64}$ Ebd. 45-46; Ankner 1977, 277; von Quillfeldt 1995, 76; Wüstemann 2004, 215 Anm. 9. 

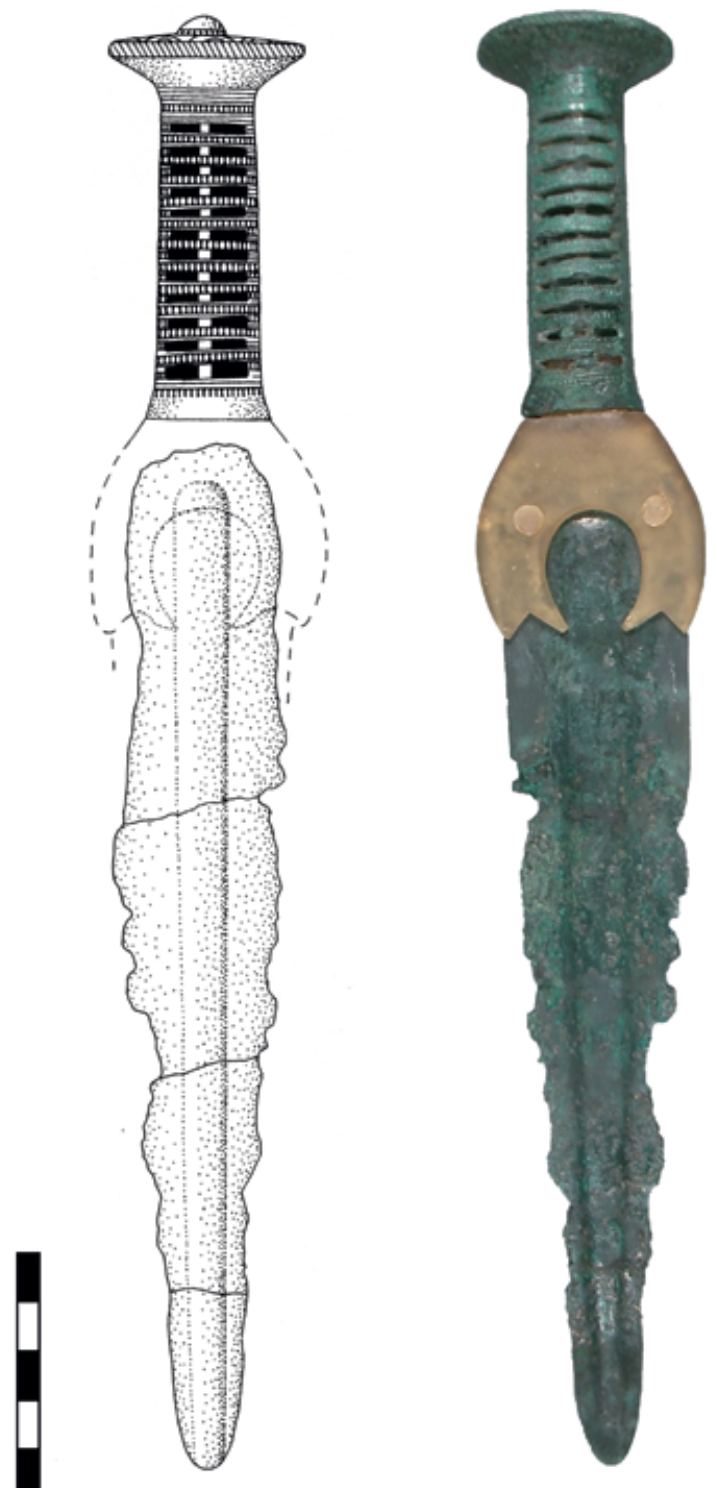

Abb. 19. Zeichnung und Foto des nordischen Vollgriffschwertes von Loxstedt (Zeichnung: Laux 2009, Taf. 31,197; Foto: S. Schwenzer)

Das aus typologischer Sicht zwischen dem Typ Spatzenhausen und den Achtkantschwertern stehende Schwert von Wedel (Nr. 64, Abb. 13) zeigt auch bei der Schäftung eine Besonderheit. Die mit zwei Nieten am Heft befestigte Klinge endet nicht in einer halbrunden Griffplatte wie bei den Schwertern vom Typ Spatzenhausen, sie weist aber auch nicht die für die Achtkantschwerter charakteristische Griffzunge auf. Der zackig ausgebrochene Rand der Klinge endet bereits im unteren Bereich der Griffsäule und erinnert am ehesten an eine abgebrochenen Griffzunge. Die Einpassung in den Heftmund ist dennoch sehr gut gelungen. Rund um die Nietlöcher finden sich leichte Risse. Es ist offenbar zumindest versucht worden, dieses Schwert nach Art der Achtkantschwerter zu schäften, wie es bei dem sehr ähnlichen Schwert von Matrei in Tirol auch ge- lungen ist ${ }^{65}$. Dieser Befund bezeugt noch einmal den Charakter des Fundstücks als Übergangsform oder parallele Entwicklung zu den Achtkantschwertern (s.o.).

Trotz der recht einheitlichen Schäftung der Achtkantschwerter können mehrere Aspekte unterschieden werden. Im vorliegenden Fundgut variieren vor allem die relative Länge der Griffzunge und die Position der Kontaktstelle an der Zunge. Es lassen sich kurze, mittellange und lange Griffzungen erfassen, je nachdem, ob sie nicht oder ziemlich genau bis zur Mitte der Griffsäule reichen oder sogar darüber hinausgehen. Die Kontaktstelle mit der Innenwand liegt entweder im oberen oder mittleren Teil der Griffzunge,

65 Hundt 1979, 183-184. 

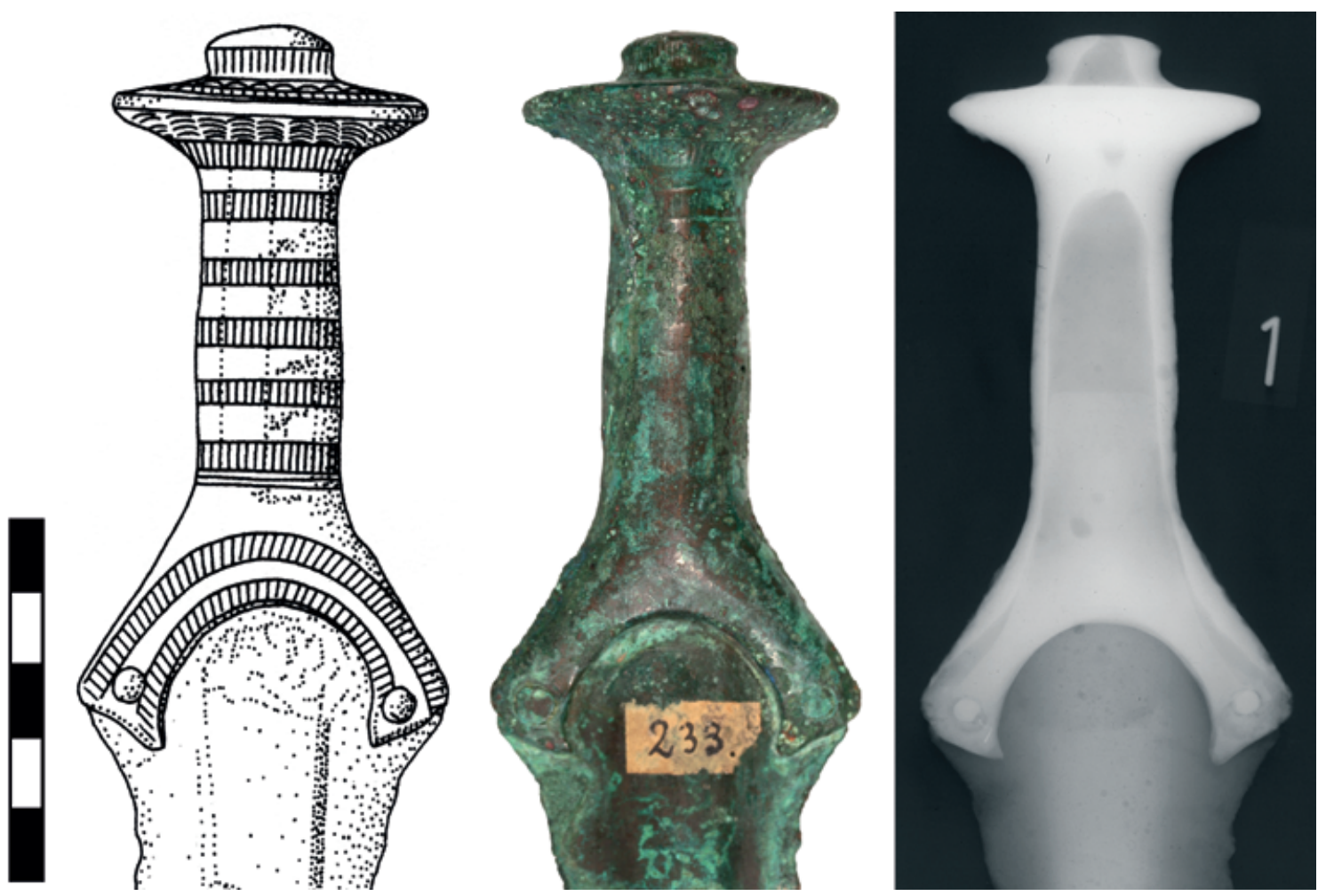

Abb. 20. Zeichnung, Foto und Röntgenaufnahme des Achtkantschwertes von Lühnenspecken (Zeichnung: Laux 2009, Taf. 25,169; Foto: S. Schwenzer;

Röntgenaufnahme: Römisch-Germanisches Zentralmuseum Mainz)

in manchen Fällen berührt auch eine längere Partie der Zunge die Wandung. Diese technische Variante ist für die Stabilität der Verbindung von großem Nutzen, da auftretende Torsions- und Scherkräfte auf eine größere Fläche verteilt werden ${ }^{66}$. Insgesamt wäre es interessant zu sehen, ob sich bei breiter angelegten radiographischen Untersuchungen aus den aufgezeigten Unterschieden gewisse Regelmäßigkeiten bei der Schäftung ableiten lassen.

Manche Achtkantschwerter zeigen eine ungewöhnlich gute Anpassung von Klinge und Heft, insbesondere die Schwerter von Ottensen (Nr. 61), Schwinge (Nr. 62, Abb. 9), aus der „Umgebung von Stade“ (Nr. 63, Abb. 11) und dem mecklenburgischen Vielist ${ }^{67}$. Bei ihnen berührt die Zunge die Innenwand im Heft und auch in der Griffsäule über weite Strecken. Möglicherweise wurden hier beide Teile speziell füreinander gefertigt. Das Schwert aus der „Umgebung von Stade“ zeigt einen deutlichen Bruch in der Griffzunge.

Andere Schwerter geben im Gegensatz dazu Hinweise auf ein mögliches Auswechseln der Klinge. Bei den Fundstücken von Lühnenspecken (Nr. 58, Abb. 20) und Meckelstedt (Nr. 59, Abb. 12) deuten deutlich ausgeweitete Nietlöcher bzw. ein versetztes Nietloch darauf hin, dass Griff und Klinge wohl nicht füreinander gefertigt wurden und

66 Wüstemann 2004, 124 Anm. 51.

67 Ebd. 121-122 Nr. 424. deshalb die Nietlöcher beim Einpassen nachgebessert werden mussten. Das Schwert von Meckelstedt, obwohl von F. Laux aus typologischer Sicht als Vorform der Achtkantschwerter charakterisiert (s.o.), offenbart bei der Schäftung keine Besonderheiten.

\section{Verzierungen und Verzierungstechniken}

Die Verzierungstechnik von bronzezeitlichen Metallobjekten wird zum Teil kontrovers diskutiert. Insbesondere ist fraglich, ob feinere Verzierungen bereits in der Gussform angelegt oder erst anschließend angebracht wurden. Untersuchte österreichische Schwerter wurden ausschließlich nach dem Guss verziert, was anhand von Aufwulstungen erkennbar ist ${ }^{68}$. E. Herner nimmt an, dass die nordischen Verzierungen aus Spiralen, konzentrischen Kreisen usw. ebenfalls nachträglich eingepunzt wurden ${ }^{69}$.

P. Rønne indes widerspricht ihr zumindest im Falle der komplexen nordischen Spiralornamentik und führt stichhaltige Argumente dafür an, dass die Ornamente stattdessen mit Drahtspiralen in die Wachsmodelle für das Wachsausschmelzverfahren eingedrückt wurden. So sind beispielsweise keine Punzspuren oder Veränderungen im Metallge-

68 Mödlinger 2007, 114; dies. 2011, 44.

${ }^{69}$ Herner 1989. 
füge festzustellen und die Spiralen einer Reihe sind in den meisten Fällen absolut identisch. Genau im Kontaktbereich zweier Spiralen befindet sich vielfach eine kleine Unregelmäßigkeit, die durch das Aneinanderreihen von Eindrücken hervorragend erklärt werden kann. Gussversuche belegen, dass es möglich ist, die zuvor eingestempelten Ornamente im Guss auf das Bronzeobjekt zu übertragen ${ }^{70}$. Eine in der Gussform bereits angelegte Ornamentik ist außerdem in solchen Fällen wahrscheinlich, in denen die Form durch das Abformen eines ebenfalls verzierten Modells hergestellt wurde ${ }^{71}$.

Trotz der jüngsten Zweifel von R. Schwab et al. sprechen diese Ergebnisse für sich ${ }^{72}$. Außerdem ist zu bedenken, dass die antiken Metallhandwerker einen weitaus größeren Erfahrungsschatz als die rezenten Nachahmer ihrer Technik hatten und deshalb eine kategorische Zurückweisung bestimmter Arbeitsweisen zumindest fragwürdig bleibt.

Obwohl also auch von mitgegossenen Verzierungen auszugehen ist, wurden viele Objekte offenbar erst nachträglich verziert. Hier muss nun diskutiert werden, welche Techniken zur Anwendung kamen. Besonders strittig ist, ob eine Bearbeitung von Bronzeoberflächen durch spanabhebende Verfahren - beispielsweise Gravieren - überhaupt mit bronzenem Werkzeug möglich ist. Falls nicht, können lediglich spanlose, das Material nur verdrängende Techniken wie Punzieren und Ziselieren verwendet worden sein. Während H. Drescher und B. Armbruster spanabhebende Verfahren unter diesen Voraussetzungen als unmöglich bezeichnen und lediglich spanlose Techniken annehmen ${ }^{73}$, ist M. Mödlinger gegenteiliger Meinung ${ }^{74}$.

Bemerkenswert erscheint die Möglichkeit, dass manche Verzierungen mithilfe von gehärteten eisernen Sticheln eingraviert worden sein könnten. Ein älterbronzezeitliches Objekt aus verhüttetem Eisen, welches aufgrund seiner Härte und Form durchaus als Stichel, Dorn oder Punze gedient haben kann, fand sich nahe Emmen in den östlichen Niederlanden im Zusammenhang mit einem Bohlenweg im Moor (dendrodatiert auf ca. 1350 v. Chr.). Kleine Mengen Eisen können bei der Kupferverhüttung als Nebenprodukte anfallen und könnten zur Herstellung von kleinen Werkzeugen gedient haben ${ }^{75}$. H.-J. Hundt rechnet mit der Verwendung von eisernen und sogar stählernen Werkzeugen in größerem Umfang ab Periode III ${ }^{76}$. Letztendlich bleiben solche Überlegungen ohne weitere Belege aber Spekulation.

Die Linien auf der Schwertklinge von Meckelfeld (Nr. 49) sind nach Drescher „,[m]erkwürdigerweise [...] allem Anschein nach gefeilt worden"77. Mit welchen Werkzeugen dies geschah, bleibt offen ${ }^{78}$. Armbruster merkt an, dass die

\footnotetext{
70 Rønne 1989, 129-142.

71 Jantzen 2008, 97, 314.

72 Schwab et al. 2007, 243-244.

73 Drescher 1956/58, 27; 1968, 138; Armbruster 2000, 56; 111.

74 Mödlinger 2007, 114; dies. 2011, 43-44.

75 Charles 1984.

76 Hundt 1968, 139.

77 Drescher 1961, 60.

78 Ebd. 59-60.
}

Herstellung dieser Linien bislang ungeklärt ist ${ }^{79}$. Mödlinger nimmt eine Gravierung oder Punzierung mit Feuerstein, Quarz oder gehärteten Bronzesticheln an ${ }^{80}$. Die Fundstücke aus Niedersachsen steuern keine neuen Hinweise bei. Während die breiteren, parallel zum Mittelgrat verlaufenden Rippen an manchen nordischen Vollgriffschwertern offensichtlich mitgegossen wurden, bleibt die Entstehung der schmalen Linienbündel bislang ungelöst.

Verzierungen finden sich im vorliegenden Fundgut abgesehen von Linien auf Klingen - nur an Griffplattenschwertern (fast ausschließlich vom Typ Sögel) und Vollgriffschwertern. Das Griffplattenschwert von Mellinghausen (Nr. 16) ist auf der Klinge mit vier Linien verziert, die auf den beiden äußeren Linien Punktreihen aufweisen. Diese Punkte wurden offenbar mit einer leicht dreieckigen Punze angebracht.

Die Vertiefungen und Durchbrüche an den Griffen diverser nordischer Vollgriffschwerter, die sicherlich Inkrustationen oder Ziermaterialien (z.B. Pech, Horn oder Bernstein) für ein abwechslungsreiches polychromes Aussehen enthielten, wurden im Guss angelegt (z. B. Abb. 5; 7; 8) ${ }^{81}$. Im Griffbereich des Schwertes von Estorf (Nr. 44) überlagert eine Dreieckspunze die mitgegossenen Rippen. Ob dieser kleine Fehler am Wachsmodell oder am fertigen Gussstück geschah, ist nicht zu klären.

Auf der Knaufplatte des Schwertes von Loxstedt (Nr. 48, Abb. 21) befinden sich sechs Spiralen, deren Inneres jeweils von einem kleinen Stift gebildet wird, wie man besonders in der Computertomographie deutlich erkennen kann. Der runde, am äußeren Rand mit kleinen Einkerbungen versehene Knaufknopf ist ebenfalls mit einem längeren Stift in die Knaufplatte eingesetzt. Weil diese Stifte offenbar keinen konstruktiven Nutzen haben, spricht das Ergebnis der Metallanalysen (s.u.) mit unterschiedlich hohen Zinnanteilen dafür, dass durch die Wahl verschiedener Legierungen ein farblicher Kontrasteffekt erzielt werden sollte. Ähnliches zeigt sich im Knauf des Schwertes von Wiepenkathen (Nr. 54, Abb. 21). Hier bilden vier kleine Stifte möglicherweise den inneren Bereich von vier der Kreisaugen auf der Knaufplatte. Diese in Niedersachsen damit zweimal in ähnlicher Form auftretende Technik ist ansonsten bei radiographisch untersuchten nordischen Vollgriffschwertern bislang nicht bekannt ${ }^{82}$. Möglicherweise wurden die einzelnen Be-

\footnotetext{
79 Armbruster 2000, 121.

80 Mödlinger 2007, 114; dies. 2011, 44.

81 Vgl. Laux 2009, 85.

82 Zuerst wurde diese Technik von Ottenjann $(1969,15)$ am Schwert von Loxstedt beobachtet. Aus mehreren Komponenten bestehende Knäufe sind sowohl aus der Schwertgruppe Hajdúsámson-Apa (Hundt 1962, 41-47; Kovács 1994, 54) als auch von „westlichen“ Vollgriffschwertern (Hundt 1962, 36-37) bekannt. Anders als die vorliegenden beiden Fundstücke haben jene Schwertknäufe jedoch eine größere Öffnung oder sogar einen oben offen gegossenen Griff, der durch eine Platte verschlossen wurde. Diese Gusstechnik kann als Weiterentwicklung des Tüllengusses bei frühbronzezeitlichen Vollgriffdolchen gedeutet werden (Hundt 1962, 45; Schwenzer 2004a, 164).
} 


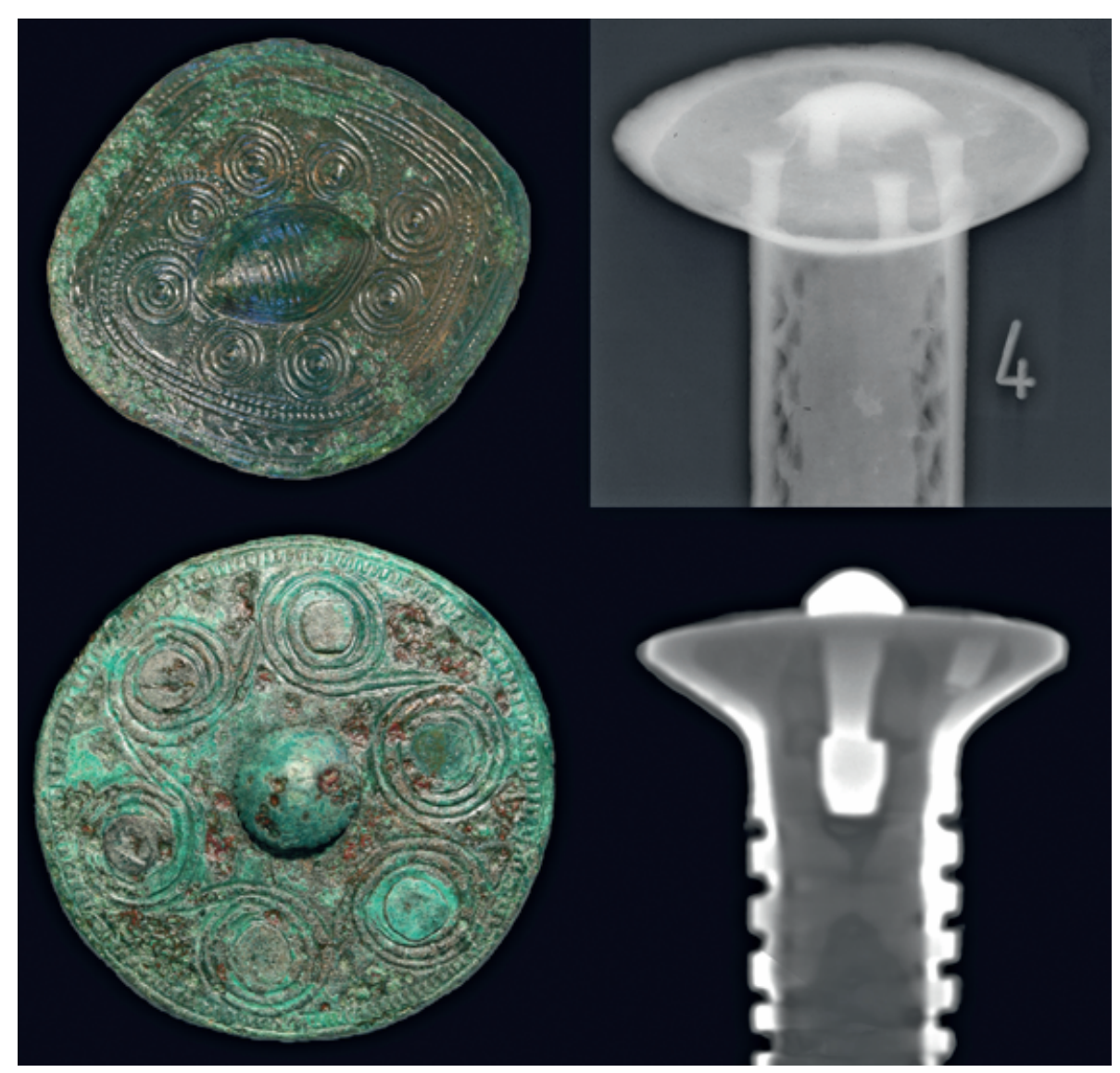

Abb. 21. Fotos und Röntgen- bzw. computertomographische Aufnahmen der Knaufbereiche der nordischen Vollgriffschwerter von Wiepenkathen (oben) und Loxstedt (unten)

(ohne M; Fotos: S. Schwenzer; Röntgenaufnahme: Römisch-Germanisches Zentralmuseum Mainz; Computertomographie: Volkswagen AG, Wolfsburg)

standteile, um die Kontrastwirkung zu verstärken, zusätzlich künstlich patiniert ${ }^{83}$.

Bei einem der Schwerter von Apensen (Nr. 41) wurde das Kreisaugen-Ornament der Knaufplatte offenbar besonders nachlässig ausgeführt und macht einen dilettantischen Eindruck. Ob es gepunzt und ziseliert oder vor dem Guss in das Wachsmodell eingearbeitet wurde, wird nicht deutlich.

Das Achtkantschwert aus der „Umgebung von Stade“ (Nr. 63, Abb. 11) wurde offenbar in zwei Schritten verziert. Zuerst war ein Girlandenmotiv auf der Knaufplatte und eine Kreisaugenverzierung am Heft vorhanden, anschließend wurden die Kanten der acht Facetten mit einer Sanduhr-Punze und die Zwischenräume mit einem V-förmigen Motiv verziert ${ }^{84}$.

Auch der Knauf des Schwertes von Wedel (Nr. 64, Abb. 13) ist in zwei Schritten verziert worden. Der ursprüngliche Knauf mit seiner vermutlich südmitteleuropäischen Ornamentik wurde mit einem Überfangguss ver-

83 Berger/Pernicka 2010.

84 Laux 2009, 74. größert und mit einer nordischen Spiralornamentik verziert, die von der älteren Griffsäulenverzierung stark abweicht ${ }^{85}$.

Das Achtkantschwert von Alfstedt, Lkr. Rotenburg (Wümme), Niedersachsen, wurde anscheinend mithilfe von drei Punzen am Griff verziert. Die Punkte, die innere Linie auf der Knaufplatte und die Kreisverzierung am Heft wurden mit einer Punze mit abgerundeter Bahn, die der übrigen Linien mit einer Schrotpunze angebracht. Punzen mit häkchenförmiger Bahn wurden für die Anbringung der Bögen auf der Griffsäule benutzt. Ob es sich um eine oder mehrere solcher Geräte handelte, ist nicht klar ${ }^{86}$.

Zusammenfassend ist zu sagen, dass es im untersuchten Fundmaterial sicher zum einen die mitgegossenen Vertiefungen und Durchbrüche im Griff sowie zum anderen nachträglich angebrachte punzierte, ziselierte und eventuell gravierte Verzierungen gibt. Inwieweit auch in Niedersachsen feine Verzierungen im Guss angelegt wurden, bleibt wegen der Korrosion an vielen Fundstücken unklar. Interessant ist die in Niedersachsen zweimal belegte Verzierung durch ein-

85 Hundt 1979, 185-186.

86 Hofmann 2003, 39. 


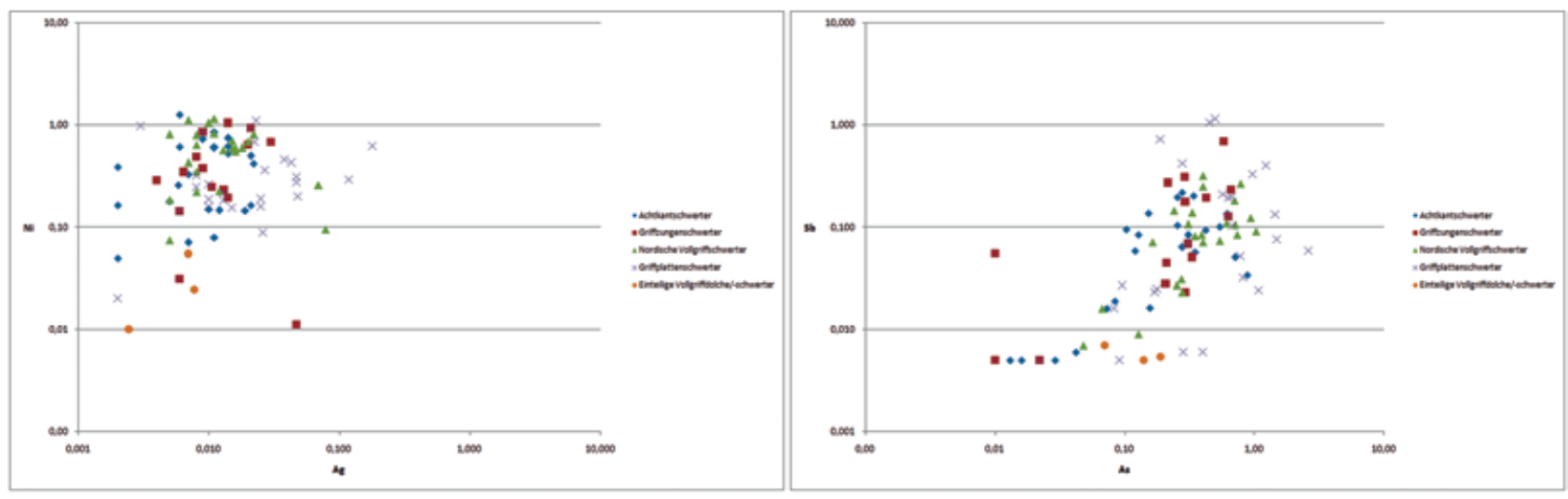

Abb. 22. Röntgenfluoreszenzanalysen-Ergebnisse: Silber/Nickel- (links) und Arsen/Antimon-Diagramm (rechts)

gesetzte Metallstifte am Knauf, die wohl eine polychrome Wirkung erzielen sollten. Dies muss - ebenso wie die Inkrustationen und Einlagen in den Vertiefungen und Durchbrüchen vieler Griffe - den nordischen Vollgriffschwertern ein prächtiges Aussehen verliehen haben.

\section{Metallanalysen}

Zur Messung der Spurenelemente kamen die Röntgenfluoreszenzanalyse (Abb. 22; Tab. 4) und für die Bleiisotopen die Massenspektrometrie mit Anregung durch ein Argonplasma und simultaner Isotopenmessung mit mehreren Kollektoren (MC-ICP-MS) zur Anwendung (Abb. 23; Tab. 1).

\section{Metallanalysen der Griffplattenschwerter}

Es wurden 20 Röntgenfluoreszenz- und zwei Bleiisotopenanalysen an Griffplattenschwertern durchgeführt, bei denen es sich in allen Fällen um Zinnbronzen mit Zinngehalten zwischen 6,1-14\% handelt.

Andere Elemente (Eisen, Cobalt, Nickel, Zink, Arsen, Silber, Antimon, Blei, Bismut) sind im Allgemeinen nur in sehr geringen Konzentrationen vorhanden. Besonders niedrige Antimongehalte finden sich in den Schwertern von Baven und Nienburg. Mit Ausnahme der Klinge von Ostereistedt (Nr. 19) mit 1,29\% Blei ist die Metallzusammensetzung als sehr bleiarm zu bezeichnen. Ob der Bleigehalt von 1,29\% auf das Erz oder das Einschmelzen von Bronzeschrott zurückzuführen ist, muss offen bleiben ${ }^{87}$. Die beiden einzigen beprobten Schwerter mit mehr als $0,1 \%$ Silber sind die Fundstücke von Klein Henstedt (Nr. 13) mit 0,119\% und Soltau (Nr. 23) mit 0,179\% Silber. Obwohl diese Werte im beprobten Material ungewöhnlich sind, lassen sie sich natürlich erklären und haben keine besonderen Auswirkungen auf die Materialeigenschaften der Legierung. Das Schwert von Osterholz-Scharmbeck (Nr. 20) zeigt einen relativ hohen Nickelgehalt von $0,98 \%$, während die

87 Pernicka 1990, 54-55.

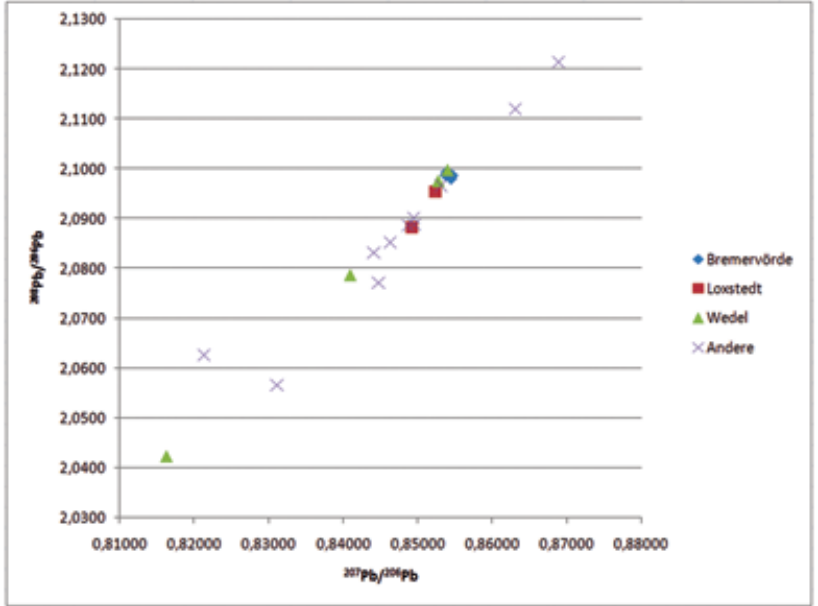

Abb. 23. Ergebnisse der Bleiisotopenanalysen

Fundstücke von Helmste (Nr. 12), Klein Henstedt (Nr. 13), Mellinghausen (Nr. 21) und Soltau (Nr. 23) vergleichsweise hohe Arsenwerte von 1,08-2,62 \% aufweisen. Arsen kann als häufiges Begleitelement von Kupfererz bei der Verhüttung als Verunreinigung unabsichtlich in das Metall gelangen. In den Schwertern von Ehestorf (Nr. 6) und Buxtehude-Altkloster (Nr. 20) befinden sich 1,15\% bzw. 0,72\% Antimon. Weiterhin gibt es einige Griffplattenschwerter, die mehr als 0,01\% Bismut enthalten. Von einer intentionellen Beimengung ist bei keinem der genannten Spurenelemente auszugehen.

Der beprobte Niet aus dem Schwert von Lemförde (Nr. 14) zeigt außer einem Zinngehalt von 5,5\% keine Besonderheiten, während das Schwert von Ostereistedt (Nr. 19) einen Niet mit einem Antimonanteil von 1,06\% aufweist.

Beim Schwert von Lemförde (Nr. 14) scheinen Schwert und Niet wegen der beinahe identischen Spurenelementgehalte aus demselben Kupfer, jedoch mit einem unterschiedlich hohen Anteil von Zinn hergestellt worden $\mathrm{zu}$ sein. Eventuell hat man für die Klinge bewusst mehr Zinn beigemengt, um die Härte zu erhöhen und möglicherweise eine andere Farbe zu erhalten. Schwert und Niet aus Osterei- 


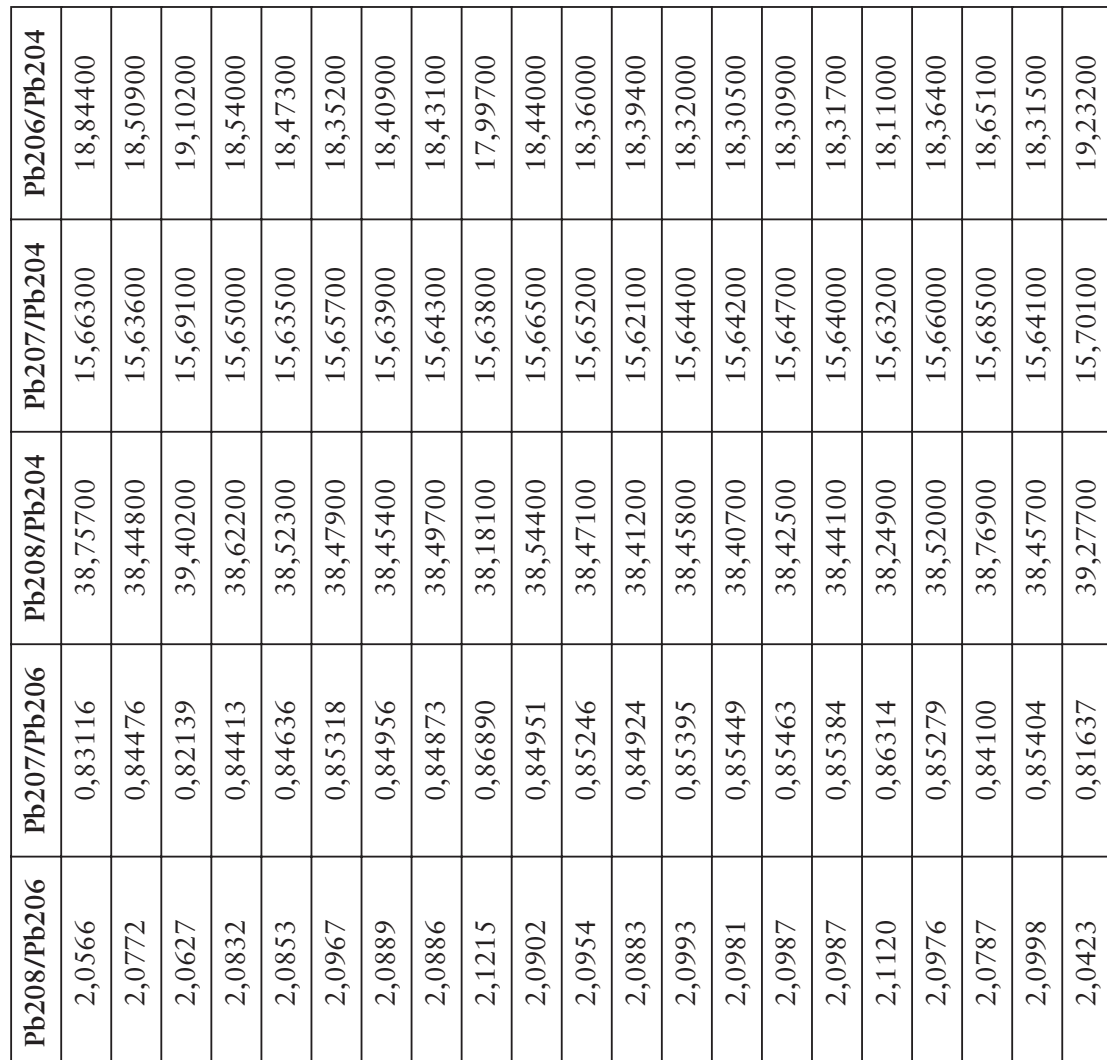

离

䇂

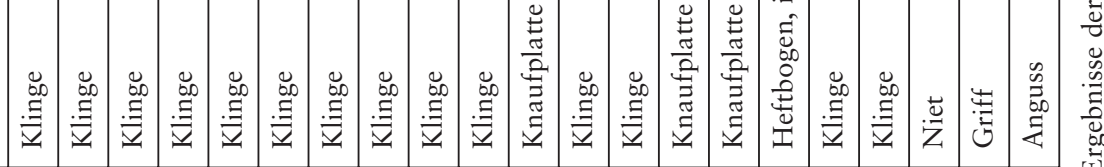

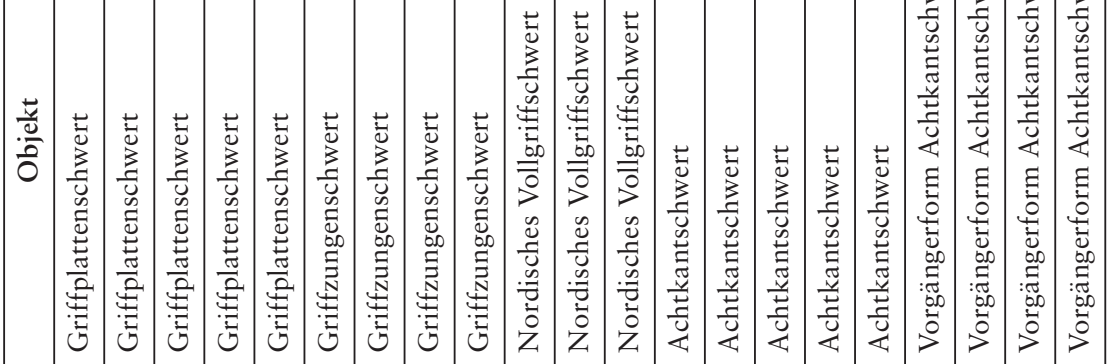

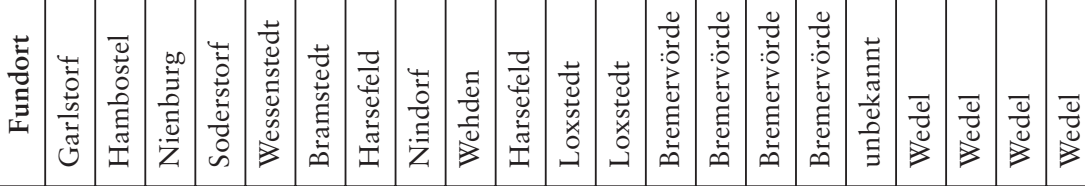

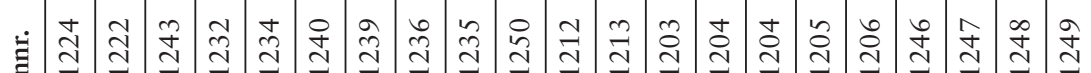

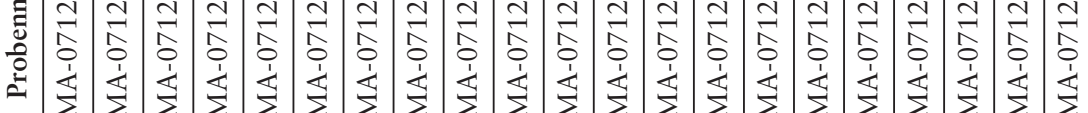

葛 
stedt (Nr. 19) hingegen sind offenbar aus unterschiedlichen Kupfersorten gefertigt, auch hier besteht die Klinge aus einer zinnreicheren Legierung.

Die für die Griffplattenschwerter verwendete Kupfersorte stimmt nach den Spurenelementkonzentrationen im Wesentlichen mit den Varianten des „ostalpinen Kupfers“ überein ${ }^{88}$.

\section{Metallanalysen der „Westeuropäischen“ Griffplattenschwerter}

Es wurden drei Röntgenfluoreszenzanalysen und drei Bleiisotopenanalysen an „westeuropäischen“ Griffplattenschwertern (sog. ,Rapierschwerter') vorgenommen. Der Zinnanteil schwankt zwischen $8,6-16,8 \%$. Die große Variationsbreite wird durch das Schwert von Wessenstedt (Nr. 28) mit 16,8\% Zinn verursacht.

Dieses Fundstück weist auch einen erhöhten Nickelanteil von $1,11 \%$ auf. Die Schwerter von Soderstorf (Nr. 22) und Wessenstedt (Nr. 28) haben zudem einen recht hohen Antimongehalt von $0,42 \%$ bzw. $0,40 \%$. Ansonsten sind andere Elemente nur in sehr geringen Anteilen nachweisbar. Insbesondere das Schwert von Garlstorf (Nr. 7) zeigt sehr geringe Konzentrationen an Nickel, Arsen und Antimon, weshalb es von den beiden übrigen beprobten Stücken abweicht. Es lässt sich nicht eindeutig entscheiden, ob es sich in dieser Gruppe um „ostalpines Kupfer“ oder Material von den Britischen Inseln handelt, da sich die Streubereiche bei den Bleiisotopenverhältnissen überlappen und die Spurenelementmuster nicht unbedingt signifikant unterschiedlich sind ${ }^{89}$.

\section{Metallanalysen der Griffzungenschwerter}

An den Griffzungenschwertern wurden 16 Röntgenfluoreszenzanalysen und vier Bleiisotopenanalysen durchgeführt. Die eigentlichen Schwerter weisen zwischen 8,3-13,5\% Zinn auf. Der Zinnanteil gruppiert sich recht eng um den Mittelwert von 11,3\%. Es handelt sich also um Zinnbronzen.

Die übrigen Elemente sind nur in sehr geringen Konzentrationen vorhanden. Besonders auffällig sind die Schwerter von Eggestedt (Nr. 31) und Farven (Nr. 32) mit extrem geringen Nickel- und Arsenanteilen und beim Stück von Eggestedt zudem einem sehr geringen Antimongehalt. Das Schwert von Bostelwiebeck (Nr. 29) zeigt extrem niedrige Arsen- und Antimonwerte.

Die Niete zeigen Zinnwerte zwischen 8,9-10\%. Das Schwert von Bramstedt (Nr. 30) hat einen Niet mit einem Eisengehalt von $1,53 \%$ und einem Bismutgehalt von 0,04\%. Der erhöhte Eisengehalt kann möglicherweise auf Korrosion zurückgeführt werden. Einen Nickelgehalt von $1,05 \%$ zeigt ein Niet des Schwertes von Langen (Nr. 35).

88 Christoforidis et al. 1988; Krause 2003, 166-169; 179 Abb. 155.

89 Vgl. Joel et al. 1997; Rohl/Needham 1998, bes. 175.
Klinge und Niet wurden beim Schwert von Nindorf (Nr. 36) möglicherweise aus derselben Charge gegossen, da die Spurenelementgehalte und der Zinngehalt nahezu übereinstimmen. Da leider nicht von beiden Teilen Bleiisotopenanalysen vorliegen, lässt sich kein eindeutiger Nachweis erbringen. Währenddessen wurden bei den Fundstücken von Bramstedt (Nr. 30) und Langen (Nr. 35) eher andere Kupfersorten für beide Teile genutzt. Ein gezielter Einsatz höherer Konzentrationen von Zinn für die Klingen lässt sich nicht nachweisen, die Schwerter von Nindorf und Bramstedt weisen sogar einen geringfügig höheren Zinnanteil bei den Nieten auf.

Der Knauf des Schwertes von Ruschwedel (Nr. 37) zeigt zwar einen ungewöhnlich hohen Zinnanteil von 23,3\%. Dieser dürfte jedoch auf einen Korrosionseffekt zurückzuführen sein, da in diesem Fall Korrosionspulver analysiert wurde.

Nach den Spurenelementen gehört das Material der Griffzungenschwerter zu den „ostalpinen Kupfersorten“.

\section{Metallanalysen der Vollgriffdolche}

Es wurden zwei einteilig gegossene Vollgriffdolche und ein solches Vollgriffschwert per Röntgenfluoreszenzanalyse untersucht. Der Zinnanteil schwankt zwischen 7,9-14,9\%. Spurenelemente sind nur in sehr geringen Konzentrationen vorhanden, insbesondere Antimon und Nickel.

\section{Metallanalysen der Nordischen Vollgriffschwerter}

An den nordischen Vollgriffschwertern wurden 23 Röntgenfluoreszenzanalysen vorgenommen, dabei wurden fünf Schwerter je dreimal beprobt (Griff, Niet, Klinge; beim Schwert von Loxstedt (Nr. 48) stattdessen Knaufplatte, ein Stift im Knauf, Klinge), zwei Schwerter zweimal (Griff, Klinge) und das Schwert von Estorf (Nr. 44; Nr. 45) viermal (Griff, Niet, Klinge, Klingenbruchstück). Außerdem wurden drei Bleiisotopien durchgeführt, davon zwei am Schwert von Loxstedt (Knaufplatte, Klinge).

Die Griffe weisen einen Zinnanteil von 10,9-16,4\% bei einem Mittelwert von 13,7\% auf. Die Knaufplatte (und damit der Griffteil) des Schwertes von Loxstedt (Nr. 48) weist die ungewöhnlich hohen Gehalte von 19,1 \% Zinn, 4 \% Eisen und $0,03 \%$ Bismut auf. Der hohe Eisenanteil kann durch Korrosion erklärt werden, möglicherweise auch der hohe Zinnanteil (es handelt sich bei der Probe um Späne mit Korrosionspulver). Das Material der eingesetzten Stifte in den Spiralen auf der Knaufplatte enthält „nur“ 15,8\% Zinn. Es kann sein, dass hier durch Farbunterschiede im Material eine polychrome Wirkung hervorgerufen werden sollte (s.o.).

Die Niete enthalten 11,7-15,8\% Zinn bei einem Mittelwert von 12,8\%. Ein Niet des Schwertes aus der „Umgebung von Stade“ (Nr. 51) weist 1,05\% Nickel auf.

Die Klingen haben einen Zinngehalt von 9,9-16,3\% mit einem Mittelwert von 13,7\%. Die einzige Klinge mit einem Zinnanteil von weniger als $11 \%$ gehört zu dem Schwert aus 
der „Umgebung von Stade“ (Nr. 51). Nicht zusammengehörig sind die beiden beprobten Klingenbruchstücke von Estorf (Nr. 44; Nr. 45), da ihre Spurenelementgehalte unterschiedlich sind (weiterhin sprechen eine unterschiedliche Klingenprofilierung und nicht zusammenpassende Bruchstellen dafür).

Besonders niedrige Arsen- und Antimongehalte finden sich bei den Fundstücken von Harsefeld (Klinge, Nr. 47), Westerwanna (Niet, Nr. 53) und Estorf (einzelnes Klingenbruchstück, Nr. 45). Letzteres weist zudem einen sehr geringen Nickelanteil auf. Mehr als 0,01\% Bismut findet sich bei den Schwertern von Westerwanna (Griff, Nr. 53), Hammah (Griff, Nr. 46), Loxstedt (Knauf, Nr. 48).

Das in Periode III datierende Schwert von Deutsch Evern (Nr. 43) zeigt in Griff und Klinge neben erhöhten Bismutwerten von jeweils $0,17 \%$ deutlich höhere Silberwerte $(0,08 \%$ bzw. $0,07 \%)$ als die übrigen, in Periode II gehörenden Vollgriffschwerter. Diese Beobachtung trifft für die nordischen Vollgriffschwerter der Periode III in Ostdeutschland aber nicht $\mathrm{zu}^{90}$.

Die verschiedenen Schwertteile wurden in Niedersachsen aus relativ ähnlichem Kupfer, aber in unterschiedlicher Legierung, gegossen. Nur selten lässt sich eine annähernde Übereinstimmung in den Spurenelementen wie bei Griff und Klinge von Wiepenkathen (Nr. 54) feststellen. Griff und Klinge von Loxstedt (Nr. 48) bestehen zwar aus relativ ähnlichem, aber nicht demselben Material, was sowohl die Spurenelementanalyse wie auch die Bleiisotopenanalyse belegen.

Mit einem Mittelwert von 13,6\% Zinn wurde für alle Schwertteile fast durchweg sehr zinnhaltige Bronze verwendet. Es wurde aber offenbar nicht gezielt für bestimmte Schwertteile ein unterschiedlich hoher Zinngehalt eingesetzt. Der Bleiwert ist in allen Proben sehr niedrig. In den Ergebnissen der Spurenelementanalyse widerspiegeln sich in erster Linie die „ostalpinen Kupfersorten“.

\section{Metallanalysen der Achtkantschwerter}

Insgesamt 24 Röntgenfluoreszenzanalysen wurden von Achtkantschwertern gemacht. Sieben Schwerter wurden je zweimal untersucht (Griff, Klinge), die Schwerter aus der „Umgebung von Stade“ (Nr. 63) und von Bremervörde (Nr. 55) dreimal (Griff, Niet, Klinge bzw. Knaufplatte, Griff [innen], Klinge) und das Schwert von Wedel (Nr. 64) viermal (Griff, Anguss, Niet, Klinge). Zudem wurden neun Bleiisotopenanalysen vorgenommen (viermal an den Schwertern von Bremervörde und Wedel, einmal an einem Schwert von „unbekanntem Fundort“ [Nr. 56]).

Die Griffe enthalten 6,9-16,9\% Zinn mit einem Mittelwert von 10,9\%. Alle Spurenelementkonzentrationen liegen weit unter einem Prozentpunkt. Ausnahmen sind Nickel mit 1,26\% beim Griff des Schwertes von Meckelstedt (Nr. 59) und Blei mit 1,33\% beim Griff des Fundstückes

90 Riederer 2004, 310-313 Tab. 24-25. von Kuhla (Nr. 57). Es muss offen bleiben, ob das Blei als Verunreinigung mit ins Kupfer gelangte oder durch das Wiederverwerten von Bronze. Der Anguss beim Schwert von Wedel (Nr. 64) enthält 14,7\% Zinn.

Bei den beiden beprobten Nieten von Wedel (Nr. 64) und aus der „Umgebung von Stade“ (Nr. 63) beträgt der der Zinngehalt 8,4 bzw. 16,8 \%.

Die Klingen weisen zwischen 7,8-12,5\% Zinn auf. Der Mittelwert beträgt $10,7 \%$. Die Spurenelemente liegen sämtlich unter einem Prozent.

Ungewöhnlich niedrige Anteile an Arsen und Antimon finden sich bei den Schwertern von Wiepenkathen (Griff und Klinge, Nr. 65), Lühnenspecken (Griff, Nr. 58) und aus der „Umgebung von Stade“ (Klinge, Nr. 63). Die Nickelgehalte sind bei den Stücken von Wiepenkathen (Griff und Klinge) und aus der „Umgebung von Stade“ (Klinge) ebenfalls sehr niedrig. Sehr niedrig sind zudem die Silbergehalte bei den Stücken von Wiepenkathen (Klinge), Lühnenspecken (Griff) und Schwinge (Klinge, Nr. 62). Ein erhöhter Bismutanteil lässt sich mit $0,03 \%$ beim Schwert von Meckelstedt (Klinge, Nr. 59) sowie mit 0,02\% bei dem von „unbekanntem Fundort“ (Griff und Klinge, Nr. 56) feststellen.

Bei einer Gruppe von Achtkantschwertern wurde für die verschiedenen Schwertteile dasselbe Kupfer mit unterschiedlich hohem Zinnanteil genutzt; so sind bei den Fundstücken von Bremervörde (Nr. 55), Schwinge (Nr. 62), Wiepenkathen (Nr. 65) und Wedel (Nr. 64) die Spurenelementgehalte der einzelnen Teile annähernd identisch. Bei letzterem stimmt nur die Zusammensetzung bei Griff und Klinge überein, während der Niet und der Nachguss aus anderem Material bestehen. Die Ergebnisse der Bleiisotopenanalysen an den Schwertern von Bremervörde und Wedel bestätigen diesen Befund exakt. Eventuell gehören auch die Schwerter von Lühnenspecken (Nr. 58), Meckelstedt (Nr. 59), Ottensen (Nr. 61) und von „unbekanntem Fundort" (Nr. 56) in diese Gruppe. Unterschiedlich ist die Materialzusammensetzung bei den einzelnen Teilen der Fundstücke von Kuhla (Nr. 57) und aus der „Umgebung von Stade“ (Nr. 63). Bei letzterem Schwert stimmt jedoch das Material des untersuchten Nietes und des Griffes überein.

Mit einem Mittelwert von 10,7\% Zinn wurde eine durchschnittlich weniger zinnreiche Legierung als bei den nordischen Vollgriffschwertern verwendet. Ebenso wie in Ostdeutschland ist die Legierung sehr bleiarm ${ }^{91}$. Zinn wurde offenbar auch bei den Achtkantschwertern nicht gezielt eingesetzt, um den speziellen Anforderungen einzelner Schwertteile noch besser zu entsprechen. Die Spurenelementkonzentrationen deuten im Allgemeinen auf die Varianten der „ostalpinen Kupfersorten“ hin.

91 Vgl. Wüstemann 2004, 126. 


\begin{tabular}{|l|c|c|c|c|c|c|c|c|c|c|c|}
\hline Objekttyp & $\mathrm{Fe}$ & $\mathrm{Co}$ & $\mathrm{Ni}$ & $\mathrm{Cu}$ & $\mathrm{Zn}$ & $\mathrm{As}$ & $\mathrm{Ag}$ & $\mathrm{Sn}$ & $\mathrm{Sb}$ & $\mathrm{Pb}$ & $\mathrm{Bi}$ \\
\hline Achtkantschwerter & 0,18 & 0,03 & 0,43 & 88,28 & $<0,02$ & 0,27 & 0,01 & 10,68 & 0,08 & 0,10 & $<0,01$ \\
\hline Nordische Vollgriffschwerter & 0,39 & 0,03 & 0,58 & 84,78 & $<0,02$ & 0,44 & 0,02 & 13,58 & 0,11 & 0,06 & $<0,03$ \\
\hline $\begin{array}{l}\text { Einteilig gegossene Vollgriffdolche } \\
\text { bzw. Vollgriffschwert }\end{array}$ & 0,02 & 0,01 & 0,03 & 88,33 & $<0,02$ & 0,13 & 0,01 & 11,41 & 0,01 & 0,03 & $<0,01$ \\
\hline Griffplattenschwerter & 0,10 & 0,01 & 0,33 & 88,54 & $<0,02$ & 0,71 & 0,04 & 10,00 & 0,22 & 0,09 & 0,02 \\
\hline „Westeuropäische“ Griffplattenschwerter & 0,07 & 0,02 & 0,44 & 86,67 & $<0,02$ & 0,53 & 0,01 & 11,90 & 0,28 & 0,03 & $<0,01$ \\
\hline Griffzungenschwerter & 0,18 & 0,03 & 0,44 & 87,16 & $<0,02$ & 0,30 & 0,01 & 11,43 & 0,15 & 0,15 & $<0,01$ \\
\hline
\end{tabular}

Tab. 2. Mittelwerte der einzelnen Schwertgattungen

\section{Zusammenfassung der Ergebnisse}

Die Schwerter wurden allgemein aus einer Zinnbronze mit rund $10 \%$ Zinnanteil hergestellt. Es handelt sich somit um eine Legierung, die gut für Nachbearbeitung durch Dengeln und Zwischenglühen geeignet ist und hohe Härtesteigerungen ermöglicht, ohne dass das Material spröde wird und reißt ${ }^{92}$. Neben dem Zinn hat man die übrigen Elemente wohl nicht intentionell zulegiert, stattdessen sind sie durch das Ausgangserz oder metallurgische Prozesse bedingt ${ }^{93}$. Die Legierungen aller in Niedersachsen beprobten Schwerter sind relativ ähnlich und lassen keine deutlichen Gruppierungen erkennen, wobei sich durchaus einige Abweichungen feststellen lassen (Tab. 2).

Während sich die Abweichungen bei Arsen, Silber und auch Blei allgemein mit Ausreißern bei einzelnen Fundstücken erklären lassen, ist doch auffällig, dass die Vollgriffdolche/Kurzschwerter bei Eisen, Nickel, Arsen, Antimon sowie Blei die Durchschnittswerte der übrigen Schwerter noch unterschreiten. Die „Rapierschwerter“ zeigen geringe Eisen- und Bleianteile und die Achtkantschwerter einen geringen Antimongehalt. Deutlich ist ebenfalls, dass die nordischen Vollgriffschwerter den höchsten Zinngehalt haben.

Als Herkunftsgebiet des Kupfers kommt nach den Spurenelementmustern am ehesten der Ostalpenraum, wahrscheinlich das Revier von Mitterberg im Bundesland Salzburg, Österreich, in Frage. Diese Lagerstätte ist durch eine einheitliche chemische Zusammensetzung und sehr variable Bleiisotopenverhältnisse gekennzeichnet ${ }^{94}$. Die Materialherkunft der „westeuropäischen“ Griffplattenschwerter, also inwiefern möglicherweise tatsächliche Importe z.B. von den Britischen Inseln vorliegen, lässt sich nicht eindeutig klären.

Eine chronologische Auswertung innerhalb der hier vertretenen Perioden I b bis III erbringt interessante Ergebnisse. Der Mittelwert für Zinn beträgt $10 \%$ für Periode I $\mathrm{b}$, $11,9 \%$ für Periode II und 12,4 \% für Periode III. Er steigt also kontinuierlich leicht an; möglicherweise ein Zeichen dafür, dass im Laufe der Zeit mehr Zinn im Umlauf war. Allerdings kann auf Basis der Untersuchung nur einer, noch

\footnotetext{
92 Vgl. Mödlinger 2007, 104; 2011, 29.

93 Krause 2003, 201-204.

94 Pernicka et al. 2008, 335-336.
}

dazu relativ speziellen Fundgruppe natürlich kein allgemeiner Trend postuliert werden.

Demgegenüber nehmen die Mittelwerte für Arsen und Antimon chronologisch kontinuierlich ab (As von $0,74 \%$ in Periode I b über $0,36 \%$ in Periode II auf $0,122 \%$ in Periode III; Sb von $0,23 \%$ in Periode I b über $0,117 \%$ in Periode II auf $0,047 \%$ in Periode III), was belegen könnte, dass später reineres Kupfererz genutzt wurde. E. Pernicka vermutet, dass entweder andere Lagerstätten, die recht reinen Kupferkies führen (z.B. Kitzbühel, Bezirk Kitzbühel, Tirol, Österreich), hinzugekommen sind oder andere Lagerstättenbereiche im Raum Mitterberg genutzt wurden ${ }^{95}$.

Am Untersuchungsmaterial lässt sich nicht belegen, dass für einzelne Schwertteile regelhaft unterschiedliche Legierungen mit höheren oder niedrigeren Zinnanteilen genutzt wurden. Dasselbe Ergebnis zeigt sich bei den ostdeutschen Schwertern ${ }^{96}$. Beim Knauf des nordischen Vollgriffschwertes von Loxstedt (Nr. 48) und den darin eingesetzten Bronzestiften besteht jedoch die Möglichkeit, dass aus ästhetischen Gründen eine Legierung mit unterschiedlichen Zinngehalten gewählt wurde.

Die Analysen einiger Fundstücke demonstrieren die Verwendung desselben Kupfers, aber mit leicht unterschiedlichen Zinnanteilen, für die verschiedenen Teile eines Schwertes. Beispiele sind das Griffplattenschwert von Lemförde (Nr. 14), das Griffzungenschwert von Nindorf (Nr. 36), das nordische Vollgriffschwert von Wiepenkathen (Nr. 54) sowie mehrere Achtkantschwerter (Nr. 55; Nr. 62; Nr. 64; Nr. 65; evtl. auch Nr. 56; Nr. 58; Nr. 59; Nr. 61). Die vorliegenden Bleiisotopenverhältnisse von drei Fundstücken (Nr. 48; Nr. 55; Nr. 64) bestätigen die aufgrund der Spurenelementanalysen gemachten Vermutungen exakt. Da es sich aber wegen unterschiedlicher Zinnanteile nicht um dieselbe Charge - das heißt eine exakt gleiche Legierung mit identischen Spurenelementmustern und Bleiisotopen handelt, wurden die einzelnen Teile jedoch in verschiedenen Arbeitsschritten gegossen.

In welchem Maße Primärbronze und Bronzeschrott zur Herstellung der Schwerter genutzt wurden, kann nur vermutet werden. Da es sich um eine allgemein relativ homogene Zusammensetzung der Spurenelemente handelt, ist die

95 Freundl. Mitt. Prof. Dr. E. Pernicka, Tübingen/Mannheim. 
Verwendung von Primärbronze wahrscheinlich. Eventuell können die vereinzelten Fundstücke mit etwas abweichender Metallzusammensetzung durch die Verwendung von wiedereingeschmolzener Bronze erklärt werden ${ }^{97}$. Dennoch sollte berücksichtigt werden, dass ein relativ homogen erscheinendes Spurenelementmuster auch bei einem selektiven Recycling von Altmaterial, wenn also gezielt ähnliches Material zusammengeschmolzen wird, erhalten bleibt ${ }^{98}$.

Der Vergleich mit den Metallanalysen der Schwerter aus Ostdeutschland zeigt, dass sich bei den Achtkantschwertern und nordischen Vollgriffschwertern die gleichen Ergebnisse abzeichnen ${ }^{99}$. Die Legierungen weisen allgemein nur geringe Mengen an Spurenelementen auf und entsprechen sich in Niedersachsen und Ostdeutschland weitestgehend. Auffällig ist jedoch, dass in Niedersachsen der Durchschnittswert für Zinn bei Achtkantschwertern und nordischen Vollgriffschwertern der Periode II um ca. 1,5\% höher liegt als in Ostdeutschland.

Für die Schwerter aus Ostdeutschland wurden zwei Materialgruppen aufgestellt ${ }^{100}$. Eine gute Einordnung der niedersächsischen Ergebnisse in diese Gruppen gelingt nicht, da die Grenzwerte von relativ vielen Fundstücken unteroder überschritten werden. Falls sich diese Materialgruppen tatsächlich auf Erze zurückführen lassen101, wurden möglicherweise nicht genau dieselben Erzvorkommen genutzt.

Leider erlaubt die Tatsache, dass für Achtkantschwerter und nordische Vollgriffschwerter sehr ähnliche Legierungen und vermutlich Kupfererze von ähnlicher Herkunft genutzt wurden, keine Aussage zur Herkunft der Achtkantschwerter im Norden, also ob es sich um importierte oder im Norden nach südmitteleuropäischer Technik gefertigte Stücke handelt (s.u.) ${ }^{102}$.

\section{Handwerksorganisation}

\section{Entwicklung und Tradition der Herstellungstechnik der Vollgriffschwerter}

Im Folgenden soll der Frage der chronologischen Entwicklung und der Abhängigkeiten der Herstellungstechniken mittelbronzezeitlicher Vollgriffwaffen von frühbronzezeitlichen Traditionen nachgegangen werden. Dabei nimmt die Aunjetitzer Kultur in der mitteleuropäischen Perspektive der Bronzezeitforschung im Hinblick auf die Entwicklung von Gusstechniken in der frühen Bronzezeit traditionell eine zentrale Stellung ein. Zahlreiche Funde belegen den Beitrag der Aunjetitzer Metallhandwerker zur Herausbildung bestimmter Gusstechniken und technischer Tradi-

\footnotetext{
97 Ebd. 283.

98 Freundl. Hinweis Prof. Dr. M. Bartelheim, Tübingen.

$99 \mathrm{Zu}$ den Metallanalysen aus Ostdeutschland siehe Riederer 2004.

100 Ebd. 280.

101 Ebd. 281; 289.

102 Ebd. 262-263.
}

tionen. Dass der ursprüngliche Antrieb dennoch keine autochthone Entwicklung in Mitteleuropa oder der Aunjetitzer Metallhandwerker war, sondern vielmehr darauf beruhte, Formen und Typen zu importieren und in eigener Technik umzusetzen, dabei aber vermutlich auch fremde Gusstechniken zu adaptieren, wurde an anderer Stelle ausführlich dargelegt ${ }^{103}$.

Ein bezeichnendes Beispiel soll an dieser Stelle dennoch noch einmal hervorgehoben werden. Es handelt sich um den Hortfund von Horoměřice, Stadt Prag, Tschechische Republik, dessen Bedeutung für die Chronologie der Entwicklung in der frühbronzezeitlichen Gusstechnik und der Zinnmetallurgie lange Zeit nicht ausreichend beachtet wurde ${ }^{104}$.

In diesem aus einem Kerngebiet der Aunjetitzer Kultur stammenden Hort ist ein Dolch, dessen Importherkunft aus Italien außer Frage steht (Abb. 24a), mit mehreren zweifellos lokal, also von den Bronzegießern der Aunjetitzer Kultur hergestellten Stücken vergesellschaftet. Unter diesen lokal hergestellten Stücken ist nun eines, das zweifelsfrei eine Nachahmung des italischen Importstückes darstellt (Abb. 24b). Wenn also aus den wenigen uns bekannten Fundvergesellschaftungen frühbronzezeitlicher Vollgriffwaffen auf eine chronologische Abfolge geschlossen werden darf, dann ist mit Sicherheit davon auszugehen, das in Italien bereits solche Waffen auf hohem Niveau gegossen wurden, als die Stücke aus dem Hort von Horoměřice hergestellt wurden.

Auch die Implikationen dieses Fundes für die Frage der Zinntechnologie sind wichtig. Während der italische Import hohe, differenziert eingesetzte Zinnanteile aufweist (Klinge $10 \%$; Griff $3 \%$ ), ist bei den Stücken der Aunjetitzer Metallhandwerker zwar ebenfalls teilweise ein differenzierter Einsatz von Zinn nachweisbar, jedoch mit deutlich niedrigeren Anteilen. Die gleichzeitig nachweisbaren, zum Teil hohen Anteile anderer Elemente wie Arsen und Antimon scheinen dafür zu sprechen, dass im Raum der Aunjetitzer Kultur mit dem längeren Überleben einer Fahlerzmetallurgie zu rechnen ist, bei der die Härte und das Aussehen der Stücke nicht durch Beimengung von Zinn, sondern durch die Verwendung von Fahlerzmetallen beeinflusst wurde ${ }^{105}$.

Für ein derart differenziertes Bild verschiedener Technologiekreise spricht auch eine Gesamtbetrachtung des Verbreitungsmusters der Zinnanteile frühbronzezeitlicher Vollgriffdolche. Im Norden und Osten dominieren Stück ohne oder mit geringeren Zinnanteilen, im Süden solche mit höheren Zinngehalten (Abb. 25). Wenn Funde wie jener von Horoměřice und andere Überlegungen eine chronologische Deutung im Sinne einer Älterdatierung jener Exemplare mit niedrigem Zinnanteil verbieten, bleibt letztlich nur die oben

103 Schwenzer 2004b.

104 Ders. 2004a, Kat. Nr. 308-313 Taf. 94-97. Zudem wurde der Fund ausführlich monographisch publiziert von Divac/Sedláček 1999.

105 Vgl. dazu auch Schwenzer 2009a, 85 ff. 

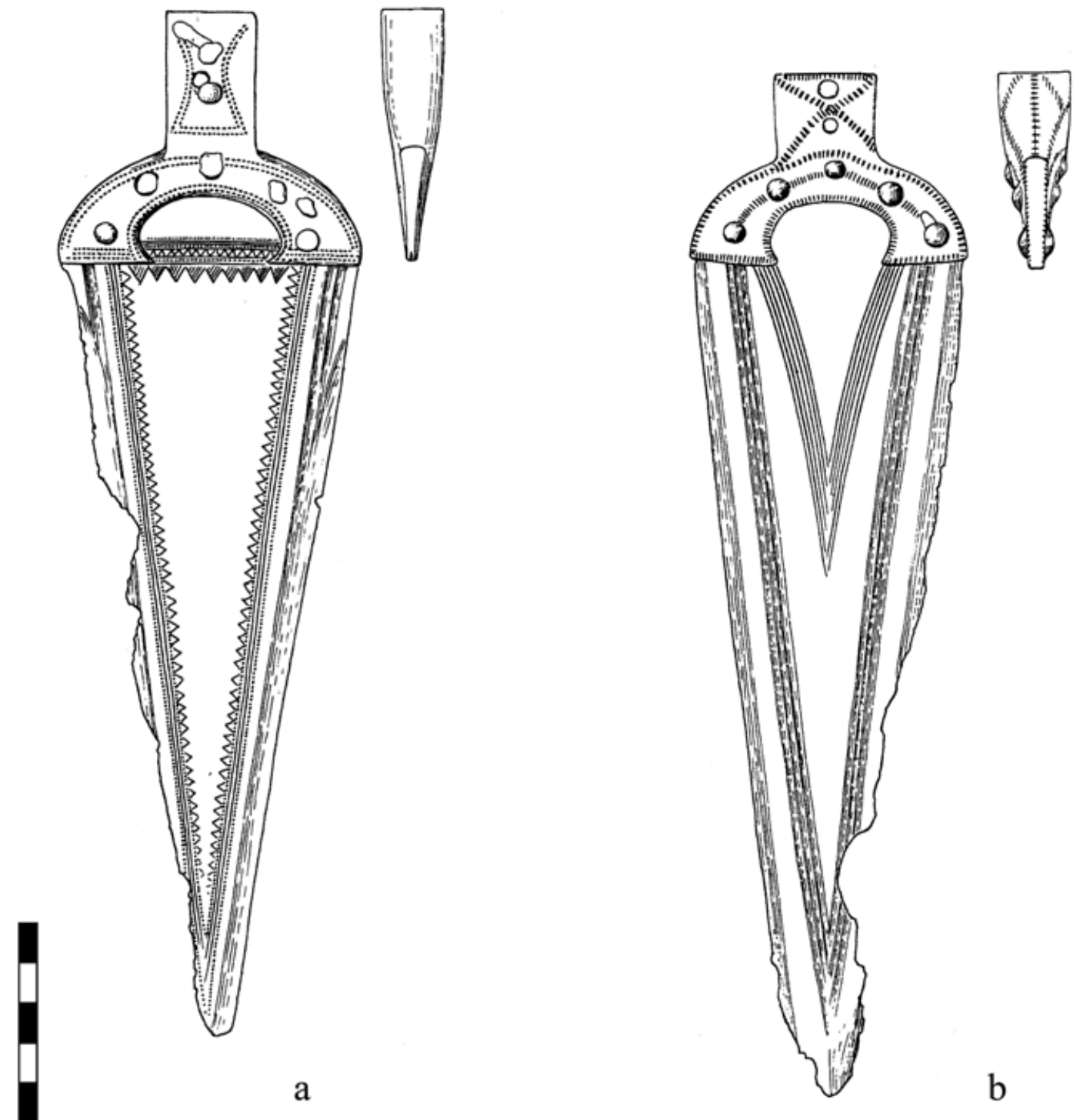

Abb. 24. Vollgriffdolche aus dem Hortfund von Horoměřice (nach Schwenzer 2004a, Taf. 96,312 u. Taf. 97,313)

genannte Annahme, dass es sich eben um unterschiedliche Technologiekreise mit einer weitestgehenden zeitlichen Überlappung handelt.

Was aber geschah mit jenen technologischen Traditionen am Ende der Frühbronzezeit? Welchen Weg nahmen die Wissenstraditionen der Metallhandwerker am Übergang zur Mittelbronzezeit? Die Aunjetitzer Kultur findet insbesondere in ihren nördlichen Verbreitungsräumen ein in prähistorischen Maßstäben relativ abruptes Ende ${ }^{106}$. Doch das Wissen und die Techniken der Metallhandwerker sind damit nicht verloren gegangen.

Es sind in der Frühbronzezeit verschiedene Gusstechniken für Vollgriffdolche nachzuweisen. Neben einteilig und mehrteilig gegossenen Griffen sind Überfangguss und der Guss ganzer Stücke in einteiligen oder mehrteiligen Formen

106 Vgl. hierzu ders. 2004a, 210f. sowie die Arbeiten von Zich 1996 und Bartelheim 1998. geläufig ${ }^{107}$. In den Aunjetitzer Funden stechen aus technologischer Sicht zwei Stücke hervor, bei denen der Griff bzw. Teile des Griffes durchbrochen gegossen wurden. Es handelt sich um ein Exemplar aus dem oben bereits angesprochenen Hort von Horoměřice und um ein Stück aus dem Hortfund von Bresinchen, dessen Knauf durchbrochen gegossen ist ${ }^{108}$. Beide Stücke können technisch als Vorläufer jener Gusstechnik gelten, die in der Periode II in großem Maßstab bei den Vollgriffschwertern des nordischen Kreises zur Anwendung kam ${ }^{109}$.

Eine weitere Technik, bei der der Griff in zwei getrennten Hälften gegossen wurde, ist ebenfalls charakteristisch für

107 Schwenzer 2004a, $141 \mathrm{ff}$.

108 Ebd. Taf. 94,308; Taf. 6,18.

109 Z.B. die im Rahmen dieses Projektes untersuchten Stücke von Loxstedt (Nr. 48, Abb. 19) und Umgebung von Stade (Nr. 51, Abb. 8). 

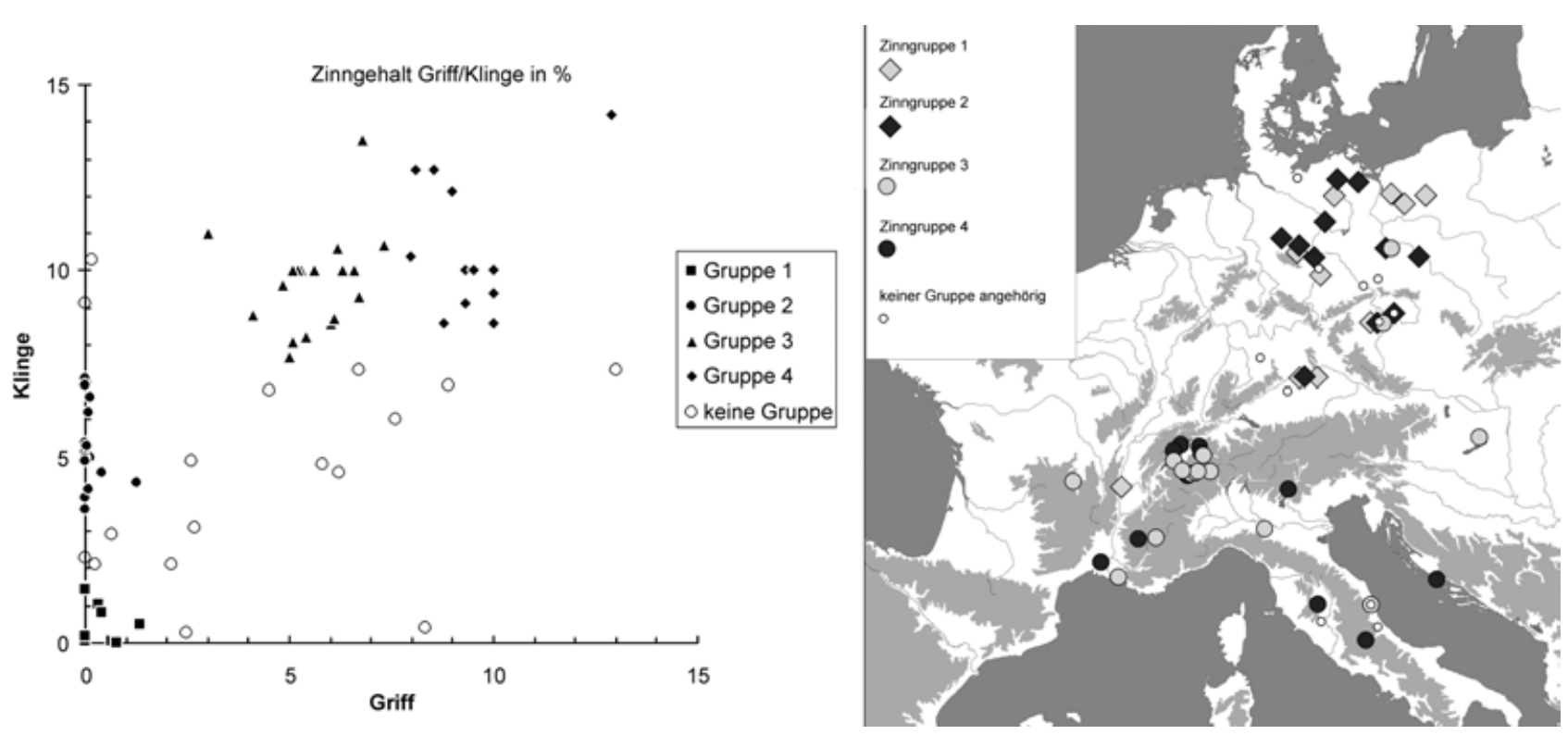

Abb. 25. Zinnanteile in Griff und Klinge frühbronzezeitlicher Vollgriffdolche. Links: grafische Darstellung der Gruppen. Rechts: Verbreitung (Schwenzer 2009a, Abb. 18 u. 19)

einen Dolchtypus der Frühbronzezeit ${ }^{110}$. Vieles spricht dafür, für diese Technik eine Herkunft aus dem norditalischen Raum anzunehmen. Am Übergang zur Mittelbronzezeit ist jene Technik nur noch vereinzelt bzw. in abgewandelter Form nachweisbar. Die beiden Vollgriffschwerter aus dem Fundensemble von Nebra sind mit jeweils einer metallenen Griffhälfte ausgestattet, ebenso ein Schwert aus dem dänischen Roum, Ksp. Felsø, Viborg Amt, sowie ein Schwert aus einem mittelbronzezeitlichen Grabfund von Rastorf, Lkr. Plön, Schleswig-Holstein ${ }^{111}$. Auch bei zwei frühmittelbronzezeitlichen Vollgriffwaffen aus Ungarn ist diese Technik noch zu finden ${ }^{112}$. Die Waffen zeigen zudem auch in der Verzierung Verwandtschaft mit Vollgriffdolchen aus dem Raum der Aunjetitzer Kultur ${ }^{113}$. Abermals zeigt sich, dass sich frühbronzezeitliche Handwerkstraditionen teils leicht abgewandelt bis in die Mittelbronzezeit fortsetzen, wenn sie sich dort auch nur noch vereinzelt wieder finden und in diesem Falle nicht dauerhaft etablieren.

Auch der Ganzguss, bei dem Schwert-/Dolchgriff und Klinge gemeinsam in einem Stück vermutlich in zweiteiligen Formen gegossen wurden, bleibt bis in die Mittelbronzezeit erhalten. Er wurde in der Frühbronzezeit insbesondere bei Dolchen des Elbe-Warthe Typs verwendet, und kann somit als eine charakteristische Gusstechnik der Aunjetitzer Metallhandwerker gelten ${ }^{114}$. In der Mittelbronze-

110 Schwenzer 2004a, $142 \mathrm{ff}$. Gruppe I. Es handelt sich um die charakteristische Herstellungstechnik der Dolche des BaltischPadanischen Typs.

111 Nebra: Meller 2002; Roum: Aner/Kersten 2008, 247 Nr. 6179 Taf. 120; Rastorf: Bokelmann 1977, 97 Abb. 8,1.

112 Vollgriffdolch von Simontornya (Kemenczei 1991, Taf. 3,9) und Vollgriffschwert von Zajta (ebd. Taf. 3,12).

113 Schwenzer 2004a, 114f. Abb. 81.

114 Ebd. 175 f. Tab. 27. zeit begegnet diese Technik nicht nur bei den zum Teil eher unscheinbaren Dolchen des Typs Lerup ${ }^{115}$ sondern auch bei einigen repräsentativen Schwertfunden aus dem Karpatenbecken ${ }^{116}$ und vermutlich auch in dem durchaus spektakulären Hortfund aus dem dänischen Dystrup, Ksp. Ørum, Randers Amt ${ }^{117}$.

Das Ende der Blütezeit des Aunjetitzer Metallhandwerkes kann mit dem Erscheinen von Vollgriffwaffen vergleichbarer Gusstechnik am Ende der Früh- bzw. dem Beginn der Mittelbronzezeit im Nordischen Kreis und im Karpatenbecken synchronisiert werden. Der Zusammenbruch des Aunjetitzer Systems setzte möglicher Weise Ressourcen und Potential frei, welche dann in anderen Regionen wirksam wurden. Ob es sich dabei lediglich um einen Wissens- und Technologietransfer handelte oder ob tatsächlich Handwerker den Aunjetitzer Raum verließen, um sich andernorts dauerhaft anzusiedeln, bleibt bisher aus Mangel an einschlägigen Befunden spekulativ.

Einen weiteren Beleg für den möglichen Transfer von gusstechnischem Wissen von der Früh- zur Mittelbronzezeit bieten die Achtkantschwerter. Die Griffe einer großen Gruppe von Vollgriffdolchen wurden wie jene der Achtkantschwerter auf einen Tonkern gegossen (Abb 26). Aber nur eine kleine Gruppe von Vollgriffdolchen aus der Westschweiz weist im Knaufbereich lunkerartige Strukturen auf, die auch bei den Achtkantschwertern beobachtet werden können (Gruppe IIb5, Abb. 27). Die Ähnlichkeiten im Befund lassen vergleichbare Bedingungen beim Herstellungs-

115 Hachmann 1957, $42 \mathrm{f}$.

116 Apa (Hachmann 1957, Taf. 63,3); Hajdusamson (ebd. Taf. 64,1); „Bezirk Satu Mare“ (Bader 1991, Taf. 8,29).

117 Rasmussen/Boas 2006. Röntgenuntersuchungen der Stücke liegen bislang leider nicht vor. 


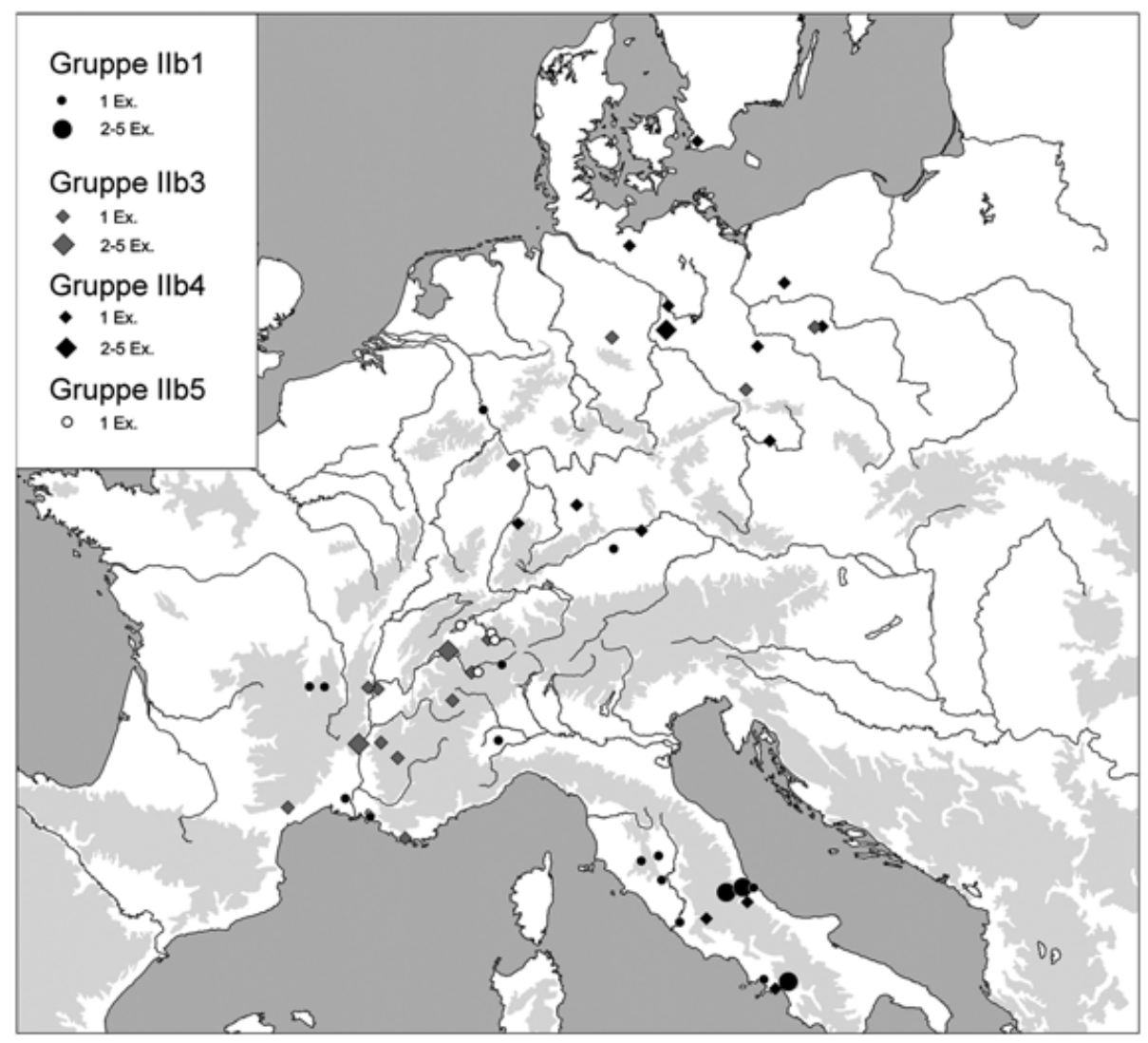

Abb. 26. Verbreitung verschiedener technischer Gruppen von Vollgriffdolchen mit auf Tonkern gegossenen Griffen (Schwenzer 2009a, Abb. 16)
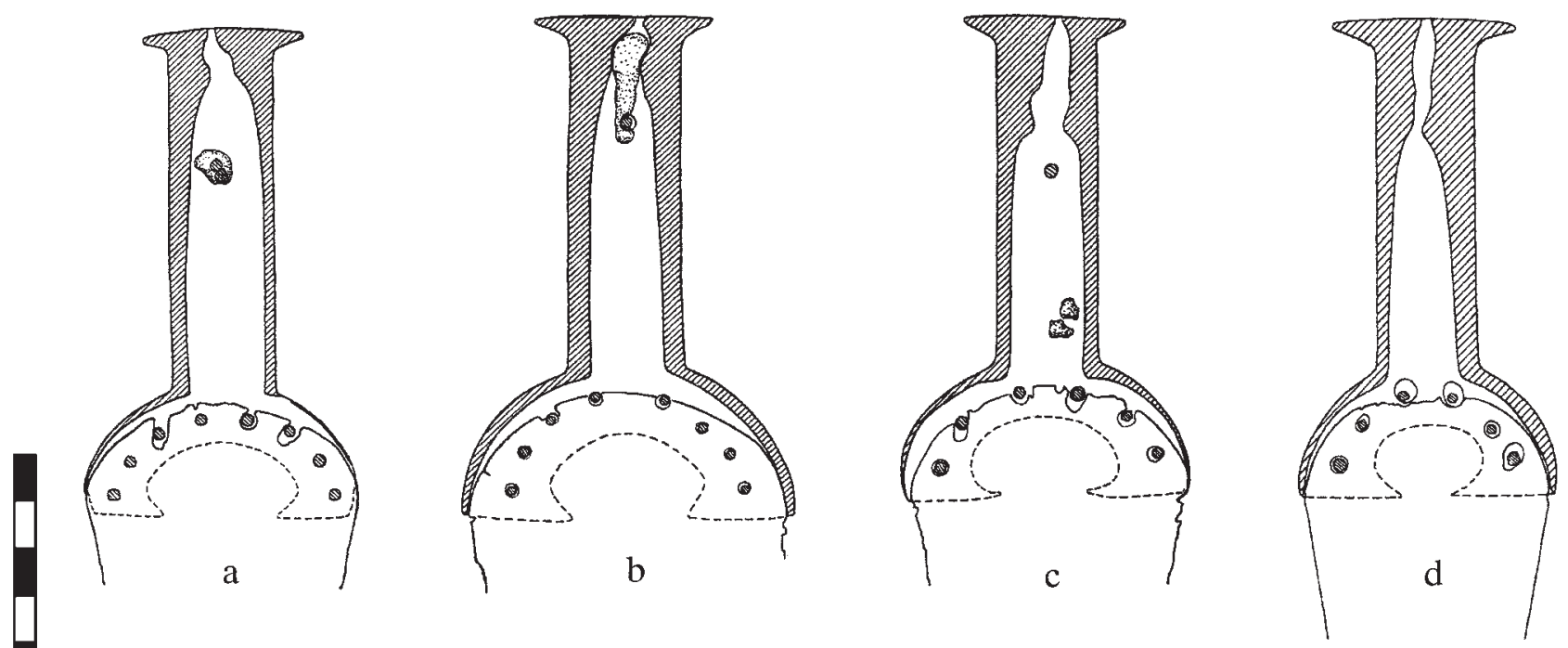

Abb. 27. Röntgenumzeichnungen von Vollgriffdolchen der technischen Gruppe IIb5

(a: Cortaillod; b: Sierre; c: Sigriswil-Ringoldswil; d: Hilterfingen) (nach Schwenzer 2004a, Taf. 85,280; Taf. 90,294 u. 295; Taf. 87,285) 
prozess vermuten. Während also im Norden Techniken der frühbronzezeitlichen Aunjetitzer Kultur in die Mittelbronzezeit tradiert wurden, könnte es im Süden ähnliche Prozesse gegeben haben, bei denen das Wissen der frühbronzezeitlichen Gießer im westalpinen Raum an die Hersteller von Vollgriffschwertern überging. Deren Herstellungszentrum vermutete schon H.-J. Hundt aufgrund ihrer Verbreitung im süddeutschen Voralpenraum, wo neben der Ressource Kupfer aus den ostalpinen Lagerstätten offenbar auch das gusstechnische Wissen verfügbar war ${ }^{118}$.

Eine Reihe von Beispielen belegt somit den Transfer technischen Wissens von der Früh- zur Mittelbronzezeit. Dabei handelt es sich um ein vielschichtiges Kommunikationsgeschehen, das neben der zeitlichen Dimension auch eine räumliche Komponente aufweist ${ }^{119}$. Die kombinierte Betrachtung von Typologie, Ornamentik und Technologie kann diese komplexen Prozesse erhellen, wie auch die folgenden Ausführungen zeigen.

\section{Beziehungen zwischen Norden und Süden}

Seit langem ist die Herkunft der Griffplattenschwerter des Typs Sögel umstritten. Obwohl sie im Einzelfall nicht voneinander unterschieden werden können, muss man wohl von Importen aus dem Mitteldonaugebiet und lokalen Nachahmungen ausgehen. Ähnliches gilt für die Schwerter des Typs Wohlde, die aus dem westlichen Ungarn hergeleitet werden können und über die Elbe und Aller nach Niedersachsen gelangten ${ }^{120}$.

Als komplex aufgebaute Kompositwaffen versprechen die Vollgriffschwerter die meisten Erkenntnisse zur Organisation und Arbeitsweise von Metallhandwerkern. Deshalb soll ihnen im Folgenden unser besonderes Augenmerk gelten.

Das Verbreitungsbild der Achtkantschwerter mit zwei räumlich getrennten Schwerpunkten im südlichen Mitteleuropa und in Nordeuropa, wobei die Schwerter der Stader Gruppe im nordöstlichen Niedersachsen die südlichsten Fundstücke darstellen, gibt seit langem Anlass zu Diskussionen (Abb. 28 links). Da sie in ihrer ganzen Erscheinung und Technik südlich geprägt sind, kam die Frage auf, ob die im Norden gefundenen Achtkantschwerter auf Import, teilweise einheimische Nachahmung oder auf süddeutsche Wanderhandwerker ${ }^{121}$ zurückgehen ${ }^{122}$. Eine weitere Möglichkeit ist der Import von unverzierten Schwertern, die erst im Norden vollendet wurden ${ }^{123}$.

\footnotetext{
118 Hundt 1965.

119 Zum Kommunikationsbegriff in der prähistorischen Archäologie vgl. Schwenzer 2009b.

120 Sicherl 1996; Laux 2009, 143-150.

121 Ausführlich zum Thema Wanderhandwerker: Neipert 2006.

122 Vgl. hierzu u.a. Holste 1953, 23; Hachmann 1956, 52-55; 1957; Hundt 1965, 47; Driehaus 1968, 360-364; Randsborg 1968, 54-55; von Quillfeldt 1995, 85-86; Wüstemann 2004, 126-127.

123 Driehaus 1968, 361; Hofmann 2003, 66.
}

Offensichtlich wurden die Achtkantschwerter - selbst wenn sie den einheimischen Vollgriffschwertern technisch keinesfalls durchweg überlegen waren - von den Menschen des Nordischen Kreises nachgefragt. Dies mag eher damit zusammenhängen, dass importierte Objekte durch ihre fremde Herkunft das Ansehen ihres Besitzers erhöhen und zeigen, dass dieser in ein Austauschsystem eingebunden ist, über welches er Kontakte aufrecht erhält, fremde Sitten kennen lernt und neue Fähigkeiten erlangt ${ }^{124}$. K. Kristiansen und T. Larsson erklären die auffälligen Verbreitungsbilder einiger Schwerttypen, darunter das der Achtkantschwerter, mit elitären „Krieger-Handwerkern“, die über Austausch- und Heiratsallianzen weiträumig miteinander vernetzt und sehr mobil waren ${ }^{125}$.

Die überaus unterschiedlichen Gusstechniken unterstreichen, dass für die Produktion von nordischen Vollgriffschwertern und Achtkantschwertern nicht die Träger derselben Metallverarbeitungstradition verantwortlich waren, also einheimische Nachahmungen sehr unwahrscheinlich $\operatorname{sind}^{126}$.

Metallanalytisch zeigt sich bei den Vollgriffschwertern jedoch kein Unterschied, es handelt sich vielmehr stets um „ostalpines Kupfer“. H. Wüstemann denkt sogar über eine Einfuhr von Achtkantschwertern als „hochwertige Metallbarren "127 nach. Tatsächlich stellt sich die Frage, warum mutmaßliche Wanderhandwerker sich den Luxus der dickwandigen süddeutschen Gusstechnik auch im Norden erlauben konnten, während die nordischen Gießer materialsparend arbeiteten. Als Lösung wurde ein Zusammenhang zwischen dem Import von Bronze und den Achtkantschwertproduzenten erwogen ${ }^{128}$. Aber auch folgende Überlegungen könnten dieses Dilemma lösen.

Die einzelnen Typen und Varianten der Achtkantschwerter zeigen eine klare Differenzierung in ihrer Verteilung auf die beiden Hauptverbreitungsgebiete. Einige Typen treten sowohl im Norden als auch im Süden auf. Dazu gehören der im Süden dominierende, aber auch im Norden häufige Typ Hausmoning, außerdem Typ Forstmühler Forst, Typ Kirchbichl, Typ Leonberg mit seiner Variante Aichach/Schrobenhausen sowie Typ Erbach. Demgegenüber gibt es eine ganze Reihe von Typen und Varianten, die nahezu ausschließlich im Norden vorkommen, nämlich der zahlreich auftretende Typ Vasby mit seiner Variante Ågerup sowie Variante Hammoor des Typs Forstmühler Forst, Variante Bedsted des Typs Hausmoning, Variante Sorgenfri des Typs Leonberg, Variante Roskilde und Variante Væggerløse Kirke des Typs Erbach, Typ Ådum, Typ Ramløse, Typ Klakring, Typ „Schonen“ und Typ Vedbæk. Im Norden zeichnet sich somit eine höhere Vielfalt von Verzierungsmotiven $a b^{129}$.

\footnotetext{
124 Kristiansen/Larsson 2005, 36-51.

125 Ebd. 231-240.

126 Hundt 1965, 47; Driehaus 1968, 360-361.

127 Wüstemann 2004, 224.

128 Mit dem Verschwinden der Achtkantschwerter beginnt nach J. Driehaus eine Materialverknappung. Eventuell hängt beides ursächlich miteinander zusammen (Driehaus 1968, 364).

129 von Quillfeldt 1995, Taf. 111B-116B; Hofmann 2003, 56-57.
} 


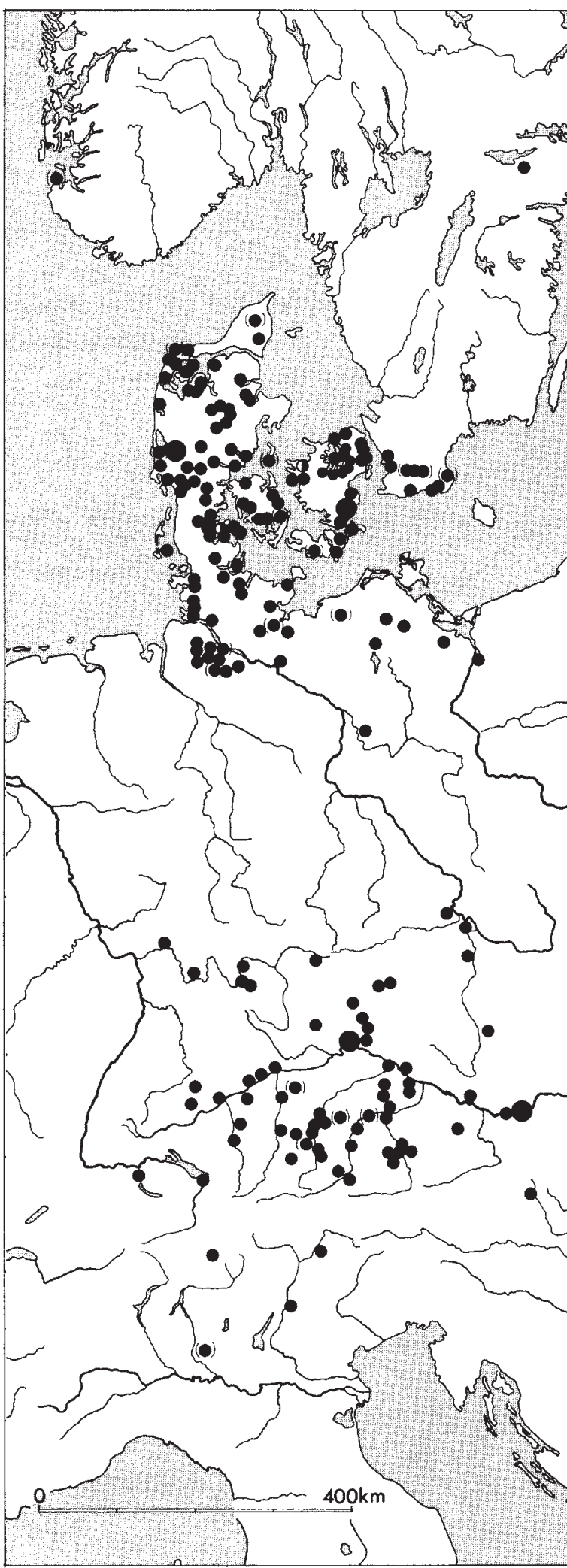

Nordische

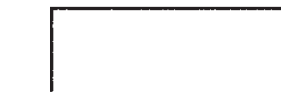

Vollgriffschwerter

selten Übernahme nordischer"

Verzierungselemente
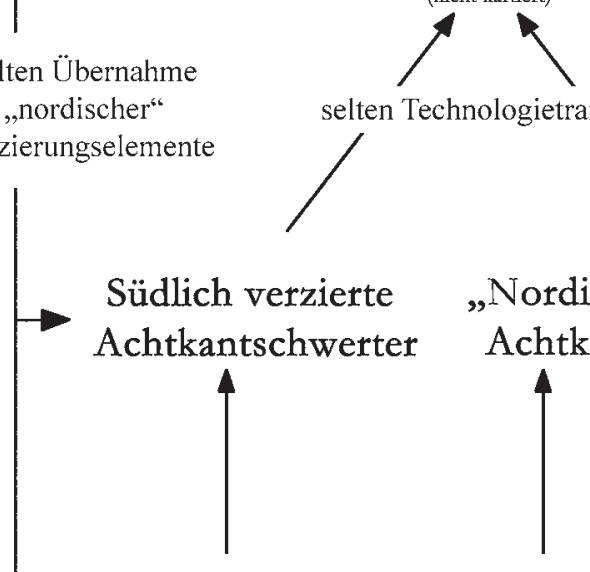

selten Technologietransfer

Nicht institutionalisierter oder institutionalisierter Import (Handel, Gabenselten Import (Handel, Gaben-

Import

tausch, Geschenke,
Beute u.v.m)

tausch, Geschenk
Beute u.v.m)

$\longrightarrow$ Verzierte

Achtkantschwerter tausch) mit anschließender Verzierung im Norden<smiles>C1CCC1</smiles>

„Nordisch" verzierte Achtkantschwerter

Unverzierte Achtkantschwerter

Abb. 28. Verbreitung der Achtkantschwerter (größere Zeichen stehen für zwei oder mehr Fundstücke) und Modell ihrer Distribution (Karte: nach von Quillfeldt 1995, Taf. 112) 
Da ein einfacher Import folglich eher auszuschließen ist, wurden die Achtkantschwerter der Typen mit nordischer Verbreitung („,nordische Achtkantschwerttypen“, im Fundmaterial: Bremervörde [Nr. 55], „unbekannter Fundort“ [Nr. 56], Lühnenspecken [Nr. 58], Ottensen [Nr. 61], Schwinge [Nr. 62]) entweder von Wanderhandwerkern aus dem Süden für nordeuropäische Auftraggeber angefertigt und auf deren Wunsch hin variantenreicher als in ihrer Heimat verziert oder die Stücke wurden unverziert importiert und erst im Norden vollendet (Abb. 28 rechts). Dass Gussstücke häufig nachträglich verziert wurden, konnte oben gezeigt werden (siehe Kapitel Verzierung). Die vornehmlich bzw. ausschließlich nordische Verbreitung einiger Typen und Varianten der Achtkantschwerter scheint auf dem ersten Blick für die Produktion durch Wanderhandwerker zu sprechen ${ }^{130}$. Da die Gruppierung der Fundstücke aber über die Griffverzierung vorgenommen wurde ${ }^{131}$, ist dieses Verbreitungsbild ebenso möglich, wenn sie unverziert eingeführt und dann im Norden vollendet wurden. Wenn diese Vermutung zutrifft, ist die oben aufgeworfene Frage, warum die postulierten Wanderhandwerker dickwandig gießen konnten, natürlich obsolet ${ }^{132}$.

Sicherlich haben sich die Handwerker aber auch von den Verzierungen der schon fertig eingeführten Stücke beeinflussen lassen, so dass nicht exakt dieselbe Ornamentik wie auf nordischen Vollgriffschwertern entstand. Gleichzeitig gibt es einige Verzierungen, vor allem am Heft, die sowohl bei nordischen Vollgriffschwertern als auch, meistens in abgewandelter Form, bei Achtkantschwertern, auftreten. Vielleicht kam es in diesem Kontext der gegenseitigen Beeinflussung auch zu der von von Quillfeldt vermuteten Entwicklung des Paragraphenmusters (kennzeichnend für den Typ Erbach mit Varianten und besonders die späteren Riegseeschwerter) aus der Spiralverzierung ${ }^{133}$. Außerdem wurden anscheinend im Zuge der Kontakte auch vereinzelte Schwerter bzw. nordische Verzierungselemente in den Süden gebracht, wie unter anderem die sehr vereinzelt vorkommenden „nordischen Achtkantschwerttypen“ oder sehr seltene Funde mit „nordischem“ Heftornament in diesem Raum zeigen ${ }^{134}$.

Andere Achtkantschwerttypen mit einem Verbreitungsschwerpunkt in Süddeutschland stellen im Norden wohl zumeist einfache Importe dar ${ }^{135}$. Beispiele hierfür sind die Schwerter des Typs Hausmoning (Kuhla [Nr. 57]) oder des Typs Erbach (Wiepenkathen [Nr. 65]). Letzteres könnte aufgrund sehr hoher Ähnlichkeit von Form und Verzierung sogar aus derselben Werkstatt stammen wie ein Fundstück aus der Gegend „zwischen Aichach und Schrobenhausen" 136 .

\footnotetext{
130 Ebd. 94.

131 Ebd. 45-46.

132 Vgl. Driehaus 1968, 361; Hofmann 2003, 66.

133 Vgl. von Quillfeldt 1995, 84; 88-89.

134 Ebd. 88; 94.

135 Vgl. Wüstemann 2004, 126.

136 von Quillfeldt 1995, 90-91.
}

Insgesamt können die im Norden gefundenen und offenbar für die dortigen Menschen begehrenswerten Achtkantschwerter damit auf zwei Hauptursachen zurückgeführt werden: zum einen auf einen Import süddeutsch verzierter Fundstücke, der nicht unbedingt institutionalisiert gewesen sein muss (denkbar sind neben Handel und Gabentausch beispielsweise Gastgeschenke und Beute), zum anderen aber auf einen Import unverzierter Schwerter in dann vermutlich institutionalisierter Form (z.B. Handel oder Gabentausch) und deren anschließende Verzierung durch einheimische Handwerker nach nordischem Geschmack (was dann die „nordischen Achtkantschwerttypen“ ergab) ${ }^{137}$. Sehr unwahrscheinlich ist wegen der unterschiedlichen Gusstechnik, dass einheimische Bronzegießer Achtkantschwerter nachgeahmt haben. Auch die vielfach postulierten Wanderhandwerker sind in größerem Ausmaß (auch mangels anderweitiger Nachweise) wohl nicht anzunehmen.

Das Verbreitungsbild der Achtkantschwerter deutet auf die Bedeutung der Elbe als Verkehrs- und Kommunikationsweg für die südmitteleuropäischen Einflüsse hin ${ }^{138}$. Es zeigt sich, dass Achtkantschwerter - ebenso wie auch nordische Vollgriffschwerter - nur auf der Stader Geest nahe der Elbmündung vorkommen. Dies hat natürlich auch damit zu tun, dass die Stader Gruppe als südlichste Grenze des Nordischen Kreises und seiner Bestattungs- und Deponierungssitten gelten muss. Dennoch scheinen unterschiedliche Grabsitten innerhalb Niedersachsens nicht allein für dieses Bild verantwortlich zu sein, da zumindest in der Allermündungs-Gruppe und in der Lüneburger Heide Schwerter ebenfalls lange zu den üblichen Grabbeigaben zählten ${ }^{139}$.

Offensichtlich übernahmen die nordischen Handwerker die ihnen durchaus bekannte und einfachere süddeutsche Gusstechnik im Allgemeinen nicht, sondern hielten an ihrer komplizierteren Arbeitsweise fest. Die Ablehnung der materialintensiveren süddeutschen Gusstechnik könnte zwar in einer Materialknappheit begründet sein, die für den Norden postuliert wird und vielleicht die Nutzung arbeitsintensiver technischer Alternativen erzwang ${ }^{140}$. Andererseits war das Metallhandwerk sicher geprägt von Traditionen und dem Erfahrungsschatz vorhergehender Generationen (siehe

137 Vgl. Kristiansen/Larsson 2005 und zum Thema Kommunikation Schwenzer 2009b, 17. Ähnlich beschreibt ein von K. Hofmann vorgelegtes Modell die Distribution der Achtkantschwerter. Sie vermutet jedoch, dass nach anfänglichem Import von süddeutschen Achtkantschwertern mit steigender Nachfrage bald Rohformen von Griff und Klinge im Norden eingeführt und von Wanderhandwerkern zusammengesetzt und nachgeschmiedet wurden. Die Wanderhandwerker oder auch einheimische Handwerker hätten die Griffe danach verziert (Hofmann 2003, 66). Meines Erachtens ist der Zwischenschritt einer erst im Norden erfolgenden Zusammensetzung jedoch unnötig - und folglich auch die Wanderhandwerker.

138 R. Hachmann $(1956,49)$ erachtet die Elbe in dieser Zeit als weniger wichtig und sieht Rhein sowie Weser als Hauptrouten. Generell unterstreichen solche Beobachtungen aber die Bedeutung der Flüsse als Kommunikationswege in der Urgeschichte.

139 Laux 2009, 140-143.

140 Driehaus 1968, 360; 363. 
auch oben) ${ }^{141}$. Die Auswahl von Technologien ist somit nicht nach rein materiellen und arbeitsökonomischen Kriterien zu verstehen. Soziale und symbolische Gründe spielen dabei ebenfalls eine entscheidende Rolle („technological choice") ${ }^{142}$.

Dennoch gibt es einige interessante Fundstücke, die die Adaption fremder Techniken erahnen lassen. Demnach scheint die dickwandigere Gusstechnik des nordischen Vollgriffschwertes von Lehsen, Lkr. Ludwigslust, Mecklenburg-Vorpommern, mit seinem massiven Knauf in einer süddeutschen Tradition zu stehen. Die ungewöhnliche Gusstechnik des Schwertes aus der „Umgebung von Stade“ (Nr. 51) könnte ebenfalls von einer fremden Tradition beeinflusst sein. Womöglich sind dies erste Hinweise auf nordische Handwerker, die vereinzelt andere Gusstechniken adaptierten oder es gab doch hier und da Wanderhandwerker aus dem Süden, die auch einmal nordische Vollgriffschwerter in ihrer eigenen Weise angefertigt haben.

Unzweifelhaft ist allerdings die Adaption der Verkeilschäftung durch nordische Handwerker, die sie wiederholt an ihren Vollgriffschwertern anwendeten, so z.B. in Estorf (Nr. 44, Abb. 16). Andere Schwerter aus Schleswig-Holstein und Mecklenburg-Vorpommern gehören ebenso in jene Gruppe (s. Anm. 57). Wie bei den nordischen Vollgriffschwertern üblich, wurde auch bei diesen Stücken zusätzlich auf eine sehr gute Einpassung ins Heft und die Vernietung mit vier bis sechs Nieten geachtet, obwohl eine solche doppelt sichere Schäftung technisch nicht notwendig gewesen wäre.

Grundsätzlich deutet sich mit den vereinzelten Adaptionen süddeutscher Guss- und vor allem Schäftungstechniken durch nordische Handwerker ein vielschichtiges Bild gegenseitiger Einflüsse und Kontakte zwischen den bronzezeitlichen Handwerkstraditionen des Nordischen Kreises und Süddeutschlands an. Auf jeden Fall können diese Aspekte von Kulturkontakten und Technologietransfer nicht nur auf die scheinbar einfache Frage nach der Distribution der Achtkantschwerter reduziert werden.

\section{Produktionsablauf}

Im Folgenden soll die Organisation der Werkstätten und eine mögliche Arbeitsteilung bei der Herstellung einzelner Teile von Vollgriffschwertern untersucht werden.

Die nordischen Vollgriffschwerter der Periode II sind nach H.-J. Hundt angesichts des Arbeitsaufwandes, der Vielfalt der verwendeten Techniken und der genauen Klingeneinpassung als Produkt der „typische[n] Arbeitsweise von Einzelmeistern" 143 anzusehen.

Im Gegensatz dazu sind die Vollgriffschwerter der Periode III in ihrer Herstellung deutlich einheitlicher. Driehaus vermutet, dass eine zunehmende Materialknappheit eine

141 Hundt 1965, 41.

142 Lemonnier 1993, 2-7.

143 Hundt 1965, 47. noch sparsamere Arbeitsweise erzwungen hat ${ }^{144}$. Die Griffangelklingen könnten unabhängig von den Griffen in einer Reihenproduktion gefertigt worden sein. Wüstemann kommt gleichwohl zu dem Schluss, dass das sehr ähnliche Material der einzelnen Teile auf eine Einzelanfertigung jedes Schwertes hindeutet ${ }^{145}$.

Für die Achtkantschwerter vermutet Hundt ausdrücklich eine „Reihenproduktion in Großwerkstätten“146. Zu diesem Schluss kommt er aufgrund der einfachen Gusstechnik und der neuartigen Schäftungsweise, die seiner Meinung nach nur in einer Großwerkstatt entwickelt worden sein kann, die die Qualität und gleichzeitig die Wirtschaftlichkeit der Abläufe verbessert hat, um die Arbeitszeit zu reduzieren und mehr Produkte herzustellen. Eine unabhängige Herstellung von abgestimmten Griffen und Klingen, die nach einer kurzen Korrektur durch Schmieden eingepasst werden können, erscheint möglich. Hundt vergleicht diese Fertigung sogar mit der modernen industriellen Produktion nach den Grundsätzen „schneller, besser, mehr“147.

$\mathrm{Ob}$ Griffe und Klingen speziell füreinander gegossen wurden, lässt sich am besten unter Berücksichtigung von Metallanalysen und der Qualität der Schäftungen untersuchen. Gussstücke mit derselben Materialzusammensetzung und eine exakt in das Heft eingepasste Klinge sprechen für eine gemeinsame Produktion ${ }^{148}$. Dennoch ist auch denkbar, dass Klingen sorgfältig passend für einen bereits existenten Griff gefertigt wurden. Hingegen lassen unterschiedliche Metallzusammensetzung und besonders eine schlechte Einpassung der Klinge eher eine separate Fertigung vermuten. Deutlich erkennbar ist eine sekundäre Verwendung der Klinge, wenn versetzte oder nicht verwendete Nietlöcher vorhanden sind. Ein sekundär genutzter Griff ist hingegen nur über die Kombination mit einer offensichtlich jüngeren Klinge eindeutig nachzuweisen ${ }^{149}$.

Für das nordische Vollgriffschwert von Wiepenkathen (Nr. 54), eine Vorgängerform und ein echtes Achtkantschwert, nämlich die Fundstücke von Wedel (Nr. 64) und Bremervörde (Nr. 55), ist durch die Kombination von Spurenelement- und Bleiisotopenanalyse nahezu sicher nachgewiesen, dass für Griff und Klinge dasselbe Kupfer, aber in einer unterschiedlichen Legierung, genutzt wurde. Für einige andere niedersächsische Achtkantschwerter (s.o.) scheint dies aufgrund fast identischer Spurenelementmuster ebenso zuzutreffen. Gleiches gilt für Griff und Klinge der nordischen Vollgriffschwerter von Havemark, Lkr. Jerichower Land, aus Sachsen-Anhalt und Retzow, Lkr. Parchim, Silmenitz, Lkr. Rügen, (alle Per. II) sowie Braunsberg, Lkr. Güstrow, und Bredentin, Lkr. Güstrow, (beide Per. III) aus Mecklenburg-Vorpommern. Alle anderen untersuchten Vollgriffschwerter in Niedersachsen und Ostdeutschland

144 Driehaus 1968, 363.

145 Wüstemann 2004, 233.

146 Hundt 1965, 47.

147 Ebd. 46-47.

148 Wüstemann 2004, 217-218.

149 Mödlinger 2007, 114; dies. 2011, 46. 
sind zwar aus einem ähnlichen, aber nicht übereinstimmenden Material hergestellt ${ }^{150}$. Insgesamt legen die Metallanalysen also für keine der beiden Vollgriffschwertformen eine regelhaft getrennte Produktion von Griff und Klinge nahe; für einige Objekte beweisen sie stattdessen sogar eine gemeinsame Herstellung beider Teile.

Für die nordischen Vollgriffschwerter ist eine gleichzeitige Herstellung von Griff und Klinge aufgrund der meist sehr gut in das Heft eingepassten Klingenenden anzunehmen. Die Fundstücke von Apensen (Nr. 41, Nr. 42) und Harsefeld (Nr. 47) haben jedoch Klingen, die wohl nicht speziell für den Griff hergestellt wurden, da ihre Einpassung in das Heft schlecht ist. Leider wurde hier nur das Schwert von Harsefeld metallanalytisch untersucht; das unterschiedliche Ergebnis für Klinge und Griff bestätigt diese Überlegungen womöglich. Auch beim Schwert von Retzow, Mecklenburg-Vorpommern, scheint die Klinge nicht für den Griff gemacht zu sein, da die Griffplatte dem Heft zwar in der Form entspricht, aber zu klein ist; dennoch scheint hier für beide Teile identisches Kupfermaterial genutzt worden zu sein ${ }^{151}$.

Bei den meisten Achtkantschwertern könnten Griff und Klinge durchaus unabhängig voneinander gefertigt und durch Ausschmieden angepasst worden sein. Bei den Schwertern von Lühnenspecken (Nr. 58) und Meckelstedt (Nr. 59) deuten deutlich ausgeweitete Nietlöcher bzw. ein versetztes Nietloch an, dass Griff und Klinge nicht füreinander gefertigt wurden und deshalb beim Einpassen eine Nachbesserung an der Klinge nötig war. Manch andere Achtkantschwerter (Ottensen [Nr. 61], Schwinge [Nr. 62], „Umgebung von Stade“ [Nr. 63] und Vielist, Lkr. Müritz, Mecklenburg-Vorpommern ${ }^{152}$ ) zeigen aber eine ungewöhnlich gute Übereinstimmung von Klinge und Heft; möglicherweise wurden hier beide Teile speziell füreinander hergestellt.

Nach den Ergebnissen der Metallanalysen und der Untersuchung der Schäftungen sind Griff und Klinge bei nordischen Vollgriffschwertern und auch bei Achtkantschwertern offenbar mehrfach aufeinander abgestimmt hergestellt worden. Eine einfache und strikte Unterscheidung zwischen von „Einzelmeistern“ gearbeiteten nordischen Vollgriffschwertern und in Serie produzierten Achtkantschwertern ist somit nicht haltbar. Die von Hundt für die Achtkantschwerter postulierten Großwerkstätten mit ihrem „modernen " Streben nach Rationalisierung und Gewinn erscheinen überdies in gewisser Weise dem Zeitgeist seiner Generation geschuldet. Eher ist von mehreren größeren und kleineren Werkstätten auszugehen, in denen das handwerkliche Wissen und die Tradition von Generation zu Generation weitergegeben wurden ${ }^{153}$. Diese Annahme bietet außerdem eine mögliche Erklärung für die zahlreichen bei den

150 Vgl. Riederer 2004, 260; 292-293 Tab. 12; 308-313 Tab. 24-25; Wüstemann 2004, 126; 224-225; 233.

151 Riederer 2004, 310-311 Tab. 24; Wüstemann 2004, 22.

152 Wüstemann 2004, 121-122 Nr. 424.

153 Brandherm/Sicherl 2001, 235.
Vollgriffschwertern vorkommenden Varianten, wobei es wiederum sehr schwierig ist zu entscheiden, wann und von wem die Verzierung angebracht wurde und wer über das Ornament bestimmte.

Da Schwerter aufgrund der langen und schmalen Form der Klingen bzw. wegen des Gusses über einem Tonkern bei den Vollgriffen sehr viel komplizierter herzustellen sind als einfachere Gebrauchsgegenstände wie Beile, kann man hier wohl von mehr oder minder spezialisierten Handwerkern ausgehen. Wie das Handwerk jedoch genau organisiert war, beispielsweise ob die Handwerker für einen „freien Markt“ oder in einem Abhängigkeitsverhältnis für eine bestimmte soziale Gruppe produzierten und ob sie ortsfest waren oder in einem gewissen Umkreis umherzogen, bleibt noch un$\operatorname{klar}^{154}$.

\section{Gebrauchsspuren}

\section{Gebrauchsspuren am Griff}

Am Griff und an den Heftschultern, die nach K. Kristiansen beim Führen des Schwertes von der Hand mit umschlossen wurden ${ }^{155}$, sind vor allem Gebrauchs- und Abnutzungsspuren in Form von abgeriebenen Ornamenten zu erwarten. Natürlich sind sie besonders wegen der Korrosion schwierig zu erfassen.

Dennoch scheint das Ornament auf den Heftschultern und am Knauf des nordischen Vollgriffschwertes aus der „Umgebung von Stade“ (Nr. 51) und auf den Heftschultern des Achtkantschwertes von Ottensen (Nr. 61) abgewetzt zu sein. Im Griffbereich finden sich auf den Achtkantschwertern von Bremervörde (Nr. 55, Abb. 10) und Lühnenspecken (Nr. 58, Abb. 20) Abnutzungsspuren. Ob die Griffsäule des Fundstücks von Wedel (Nr. 64, Abb. 13), einer Übergangsform vom Typ Spatzenhausen zu den Achtkantschwertern, intentionell einen nur angedeuteten achtkantigen Querschnitt hat oder die Facetten einfach stark abgenutzt sind, bleibt offen.

\section{Gebrauchsspuren an der Klinge}

Die Identifizierung von antiken Gebrauchs- und Abnutzungsspuren ist mit großen Schwierigkeiten behaftet. Solche Beschädigungen können - insbesondere bei gering patinierten Fundstücken - häufig nicht hinreichend datiert werden ${ }^{156}$. Sie können vor der Deponierung, intentionell anlässlich der Deponierung oder erst bei oder nach der Auffindung entstanden sein. Bei vielen Klingen verhindern ausgeprägte Korrosion oder Restaurierungsmaßnahmen bereits das Erkennen von entsprechenden Spuren ${ }^{157}$. Dennoch lassen sich mit der nötigen Vorsicht Aussagen zu alten Beschädigungen treffen. S. Bridgford hat typische Verletzungen spätbronze-

154 Jantzen 2008, 305-311; Mödlinger 2011, 33.

155 Kristiansen 2002, 320.

156 Vgl. von Quillfeldt 1995, 20-21.

157 Mödlinger 2007, 114-115; dies. 2011, 83. 


\begin{tabular}{|l|l|}
\hline Bezeichnung & Beschreibung \\
\hline Wölbung & $\begin{array}{l}\text { Deformation ohne Materialverlust; } \\
\text { Länge größer als Tiefe }\end{array}$ \\
\hline Abschlag & $\begin{array}{l}\text { Beschädigung mit Materialverlust; } \\
\text { Länge größer als Tiefe }\end{array}$ \\
\hline Scharte & $\begin{array}{l}\text { v-förmige Beschädigung ohne Materialverlust; } \\
\text { Tiefe größer als Länge }\end{array}$ \\
\hline Kerbe & $\begin{array}{l}\text { Beschädigung mit Materialverlust; } \\
\text { Tiefe größer als Länge }\end{array}$ \\
\hline Ritz & Längerer, ziemlich schmaler Einschnitt \\
\hline Riss & Riss ohne Materialverlust \\
\hline
\end{tabular}

Tab. 3. Definition von Klingenbeschädigungen (nach Bridgford 2000, Kap. 3.3)

zeitlicher Klingen in Großbritannien zusammengestellt und verschiedene Bezeichnungen definiert (Tab. 3).

Die ursprüngliche Klingenform eines Schwertes und ihr Querschnitt können durch Nachschärfung starken Veränderungen unterliegen. Die Gliederung des Querschnitts durch Rillen, Riefen und Mittelrippe verschleift sich dabei allmählich. Man kann deshalb nur bei noch vorhandener deutlicher Gliederung von einem Zustand ausgehen, der ungefähr dem Ursprungszustand der Klinge entspricht ${ }^{158}$. Das Nachschärfen bedingt den Gebrauch des Schwertes, da nur bei Schäden an der Klinge ausgedengelt oder nachgeschärft werden muss ${ }^{159}$.

Im Folgenden sollen die Gebrauchsspuren im Fundmaterial unter Verwendung der genannten Begriffe (siehe Tab. 3) vorgestellt werden.

Am Griffplattenschwert von Baven (Nr. 2) lässt sich eine Reparatur erkennen. Hier wurde ein ausgebrochenes Nietloch repariert, indem das Material unterhalb eingeschnitten und so verbogen wurde, dass der Schaden fast verschlossen ist (Abb. 29). Während das Fundstück von Lemförde (Nr. 14) nur eine minimale Verformung an der Klingenspitze aufweist, zeigt die Schneide des Fundstückes von Nienburg (Nr. 17) trotz starker Korrosion vermutlich Gebrauchsspuren. Beim Schwert von Osterholz-Scharmbeck (Nr. 20) bleibt unklar, ob die kleinen Verletzungen an der scharfen Schneide durch Gebrauch oder Korrosion verursacht sind, beim Schwert von Soltau (Nr. 23) hingegen ist eine deutliche Wölbung und ein Abschlag an der Schneide zu sehen. Das Griffplattenschwert aus der „Umgebung von Bremen“ (Nr. 27) weist möglicherweise nachträglich überarbeitete Abschläge im unteren Klingenbereich auf.

Das Griffzungenschwert von Eggestedt (Nr. 31) zeigt trotz rezentem Abschliff und Korrosion einzelne kleine

158 Kristiansen 1984, 188-195; Kristiansen 2002, 323; Thrane 2006, 495-496. Daher kann der heutige Zustand der Klinge für die Klassifikation eigentlich keine entscheidende Rolle spielen. Laux (2009) bildet seine Varianten dennoch z. T. nach dem Kriterium des Klingenquerschnittes.

159 Mödlinger 2007, 117; dies. 2011, 83-84.
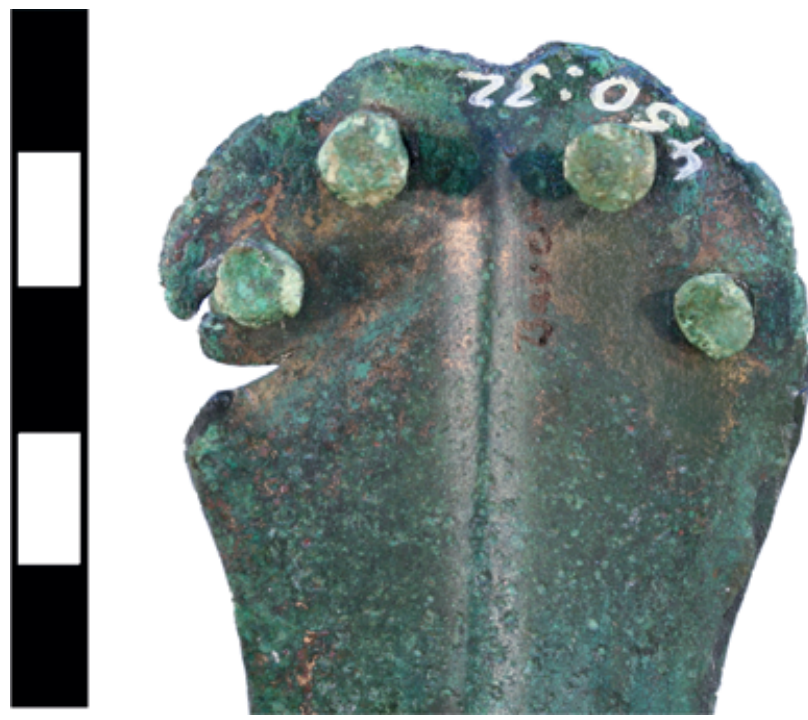

Abb. 29. Griffplatte des Schwertes von Baven (Foto: S. Schwenzer)

Wölbungen, die vermutlich auf Gebrauch hindeuten. Die Klinge des Schwertes von Harsefeld (Nr. 34) weist unregelmäßige Schneiden und kleine Ausbrüche auf. Möglicherweise liegt hier neben der Korrosion auch Nachschärfung vor. Im griffnahen Drittel der Klinge hat das Schwert von Nindorf (Nr. 36) eine unregelmäßige Klingenform, Abschläge und Scharten. Das Griffzungenschwert aus der „Umgebung von Stade“ (Nr. 39) offenbart an der Schneide und im Ricassobereich deutliche Beschädigungen, nämlich Abschläge, Scharten und einen Riss am Übergang zum Heft (Abb. 30).

Die Schneiden des nordischen Vollgriffschwertes von Apensen (Nr. 41) sind relativ gut erhalten und zeigen keine Abnutzungsspuren. Die schneidenparallelen Rillen auf der Klinge sind aber besonders im unteren Klingenteil verschliffen. Ein Nietloch fehlt zusammen mit der gesamten Ecke des Heftes. Das Klingenbruchstück von Estorf (Nr. 45) lässt an seinen korrodierten Schneiden zumindest ein paar Abschläge und eine Scharte erkennen. Die Schneide des Schwertes von Nordholz (Nr. 50) weist Abschläge und Scharten auf, während auf der Klinge des Schwertes aus der „Umgebung von Stade“ (Nr. 51) keine deutlichen Gebrauchsspuren erkennbar sind. Möglicherweise wurde sie aber häufig nachgeschliffen, da sie stellenweise bis an die Mittelrippe verschmälert erscheint und sehr dünn ist.

Nur bei einem einzigen Achtkantschwert sind - auch wegen starker Korrosion der übrigen untersuchten Stücke entsprechende Gebrauchsspuren erkennbar: das Klingenfragment des Schwertes von Bremervörde (Nr. 55) zeigt mindestens eine starke Wölbung.

Die Nietlöcher sind bei 24 Schwertern, besonders Griffplattenschwertern (16 Stück) und den „westeuropäischen“ Griffplattenschwertern (vier Stück), ausgerissen. Insgesamt 34 Schwerter sind gebrochen, davon fehlt zehn Fundstücken die Klingenspitze. Brüche treten bei Griffplattenschwertern anteilsmäßig seltener auf. Bei vier Griffzungen- 


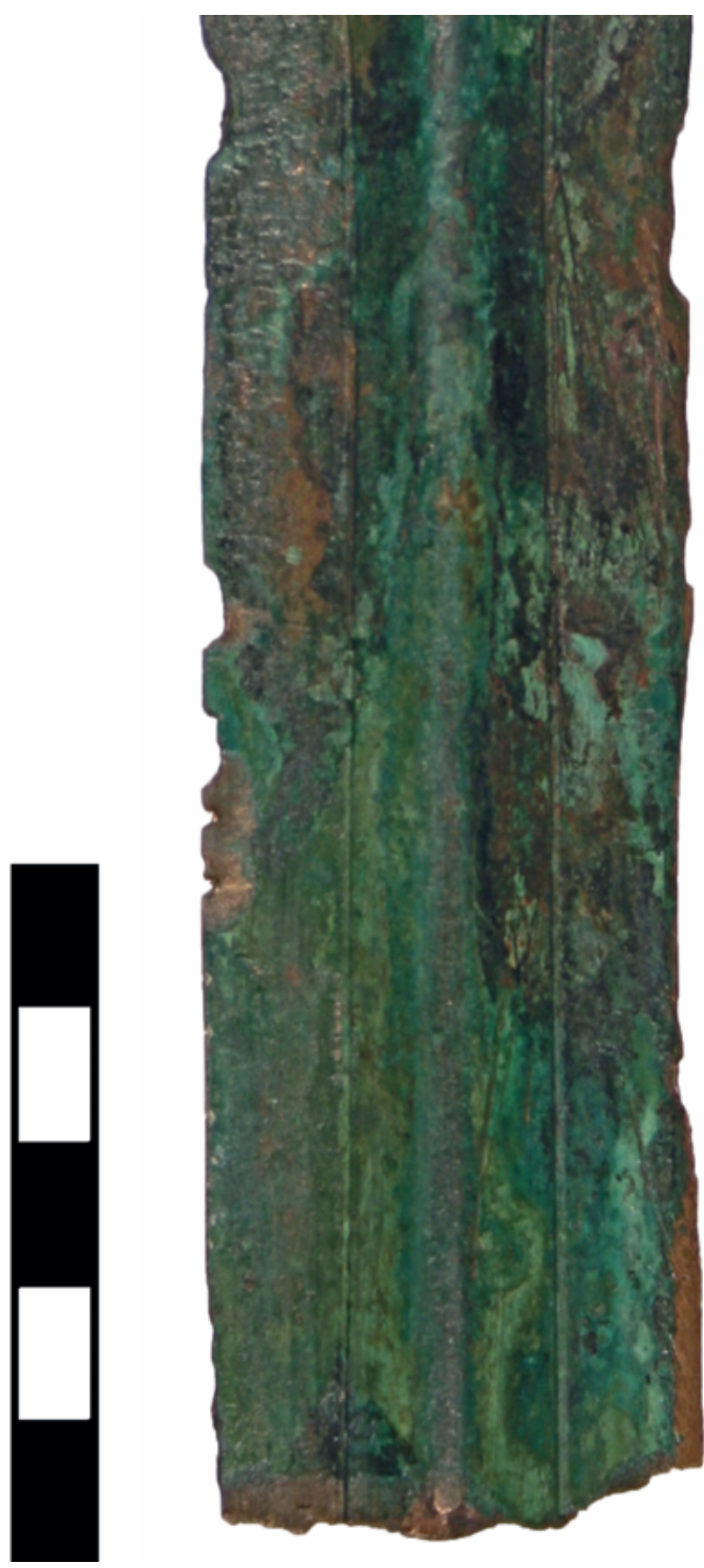

Abb. 30. Beschädigungen an der Klinge des Griffzungenschwertes aus der „Umgebung von Stade“ (Foto: S. Schwenzer)

schwertern fehlt die Zunge oder ein Teil derselben. Einzelne Brüche sind wegen der fehlenden Patina aber sicherlich auch rezent zu datieren.

In Niedersachsen finden sich kleinere oder größere Klingenverletzungen besonders an Griffzungenschwertern. Ebenfalls, aber seltener kommen sie an Griffplattenschwertern, nordischen Vollgriffschwertern und Achtkantschwertern vor. Nachschärfungsspuren wiederum sind an nordischen Vollgriffschwertern am häufigsten vertreten. Insgesamt scheinen die Vollgriffschwerter etwas weniger Gebrauchsspuren aufzuweisen als solche mit organischem Griff. Angesichts der geringen Anzahl von Untersuchungen muss man aber sehr vorsichtig mit Verallgemeinerungen sein.

\section{Funktion der Schwerter}

Die Funktion bronzezeitlicher Waffen, insbesondere der Schwerter, wird seit langem diskutiert. Im Mittelpunkt steht der (scheinbare) Gegensatz einer praktischen oder sozialen bzw. symbolischen Nutzung ${ }^{160}$. Besonders vor dem lange Zeit vorherrschenden Bild einer friedfertigen Urgeschichte wurde eine praktische Verwendung von Schwertern ignoriert bzw. bestritten ${ }^{161}$.

Als eines der bekanntesten Gegenargumente zum praktischen Gebrauch wird der vermeintlich zu kurze Griff angeführt. Wenn die Heftschulter von der Hand des Kämpfenden aber mit umschlossen wird, lässt dies ausreichend Platz für die Hand und erlaubt zudem eine gute und sichere Führung der Waffe ${ }^{162}$.

Erkenntnisse zur Funktion von Schwertern versprechen Form, Schwerpunkt, Herstellungstechnik und Gebrauchsspuren. Besonders die letzten beiden Punkte sollen hier besprochen werden. Die Form wird schon in zahlreichen typologischen Studien ausführlich behandelt ${ }^{163}$. Der Schwerpunkt hingegen ist außer bei Vollgriffschwertern wegen des fehlenden organischen Griffes schwierig zu bestimmen ${ }^{164}$.

Das Material ist durchweg eine Zinnbronze mit rund $10 \%$ Zinnanteil. Gerade bei den nordischen Vollgriffschwertern liegt der Zinnanteil mit einem Mittelwert von $13,6 \%$ besonders hoch. Von Periode I b bis Periode III nimmt der mittlere Zinnanteil kontinuierlich von $10 \%$ auf $12,4 \% \mathrm{zu}$, es wurde also im Laufe der Zeit mehr Zinn verwendet. Das Material ermöglicht durch Nachbearbeitung mittels Dengeln und Zwischenglühen eine hohe Härtesteigerung ohne spröde zu werden und spricht durchaus für eine praktische Verwendung der Schwerter als Waffen. An niedersächsischen Kurzschwertern des Typs Dahlenburg wurde bei metallographischen Untersuchungen zudem nachgewiesen, dass sie effektiv nachbearbeitet wurden ${ }^{165}$.

Bei den Vollgriffschwertern ist besonders die Schäftung ein wichtiger Faktor für die Funktionsbestimmung. Die Schäftung der meisten nordischen Vollgriffschwerter basiert in Periode II auf einer möglichst genauen Einpassung der Klinge in das Heft und mehrmaliger Vernietung, was für die wohl vornehmlich als Stichwaffen gebrauchten Schwerter ausreicht ${ }^{166}$. In Periode III weisen die Vollgriff-

160 Vgl. u.a. Kristiansen 1984, 194-195; Bridgford 2000, 105; Kristiansen 2002, 320; Molloy 2007, 108-109.

161 Vandkilde 2006.

162 Kristiansen 2002, 320.

163 Insbesondere die zahlreichen Bände zu Schwertern in der PBFReihe, Abteilung IV.

164 Mödlinger 2007, 116; dies. 2011, 86.

165 Kaufmann et al. 1996, 273-278.

166 Driehaus 1968, 362; Wüstemann 2004, 217. 
schwerter eine Griffangel auf, die durch die Griffsäule verläuft und am Knauf vernietet ist ${ }^{167}$. Auch dies kann als funktionale Lösung gelten. Die Schäftung der Achtkantschwerter ist eine wichtige Neuerung gegenüber ihren Vorgängern, die den Nutzen dieser Schwerter stark verbessert hat. Da die Griffzunge im Griffinneren verkeilt ist, wird die Gefahr eines Bruches am Heft ausgeschaltet. Die beiden Niete und die zwei Berührungspunkte von Zunge und Griff geben der Verbindung, besonders bei größeren Berührungsflächen, auch bei stärkeren Hebelbelastungen Stabilität ${ }^{168}$. Die vorliegenden Schwerter aus Niedersachsen zeigen die genannten Merkmale und können als funktionsfähig gelten. In Mecklenburg-Vorpommern dagegen wirkt die Schäftung einzelner Fundstücke instabil. Die Achtkantschwerter aus „Mecklenburg“, Alt Tellin, Lkr. Demmin, und „bei Schwaan“, Lkr. Bad Doberan, aus MecklenburgVorpommern, deren Griffzungen nicht mit der Griffinnenwand verkeilt sind, weil sie zu schmal bzw. nicht entsprechend ausgeschmiedet sind, fallen hier auf. Beim Schwert von Alt Tellin passt auch die Griffplatte nicht zum Heft. Wüstemann schlägt für diese unzureichend geschäfteten Schwerter eine Herstellung speziell als Votivgaben vor ${ }^{169}$.

Bei den nordischen Vollgriffschwertern von Apensen (Nr. 41, Nr. 42) und Harsefeld (Nr. 47) sowie den Achtkantschwertern von Lühnenspecken (Nr. 58) und Meckelstedt (Nr. 59) finden sich Indizien dafür, dass die Klinge nicht speziell für den Griff gefertigt wurde; es wäre möglich, ist aber nicht zu beweisen, dass es sich um einen Klingenaustausch handelt. Möglicherweise ist das Auswechseln alter Klingen ein Grund, weshalb Kristiansen Gebrauchsspuren seltener an Vollgriffschwertern als an Griffzungenschwertern feststellen konnte ${ }^{170}$.

Eine besonders kritische Stelle eines Schwertes liegt unterhalb der Schäftung im oberen Bereich der Klinge. Hier befinden sich bei Achtkantschwertern häufig Gasblasen, die zum Zerbrechen der Klinge führen können ${ }^{171}$. Obwohl solche Blasen die Stabilität der Klinge negativ beeinflussen, haben sie die Funktionstüchtigkeit in den Augen der bronzezeitlichen Handwerker und Kämpfer offenbar nicht erheblich beeinträchtigt, da eine bessere Qualität wohl durchaus möglich gewesen wäre ${ }^{172}$. Auch die niedersächsischen Schwerter weisen vielfach Gasblasen auf, wobei die Klingen der Achtkantschwerter besonders auffallen.

Nach Klingenform und Balance wird bei den Schwertern häufig zwischen Stich- und Hiebwaffen unterschieden. Der Begriff Hiebwaffe täuscht jedoch darüber hinweg, dass weniger wuchtige Schläge, als eher schneidende Bewegungen ausschlaggebend sind ${ }^{173}$. M. Mödlinger rekonstruiert die hauptsächliche Kampfweise über die Verteilung der Gas-

\footnotetext{
167 Wüstemann 2004, 231-232.

168 Hundt 1965, 46; Wüstemann 2004, 124 Anm. 51.

169 Wüstemann 2004, 124-125.

170 Willroth 1999, 54.

171 Driehaus 1968, 362.

172 Mödlinger 2007, 123 Anm. 72; 2011, 32-33; 93.

173 Molloy 2007, 94-95.
}

blasen, da der Angusspunkt vermutlich so gewählt wurde, dass die Bereiche mit der Hauptbelastung - je nach Funktion die Spitze oder das obere Klingenende - blasenfrei bleiben. Allerdings merkt sie gleichzeitig an, dass eine scharfe Trennung nicht sinnvoll ist, da stets beide Verwendungen möglich sind ${ }^{174}$. So wurde das niedersächsische Griffplattenschwert von Hambostel (Nr. 10), obwohl der Form nach eine Stichwaffe, dieser Logik folgend eher als Hiebwaffe gegossen. Bei den untersuchten „westeuropäischen“ Griffplattenschwertern und knapp einem Drittel der Griffplattenschwerter aus Niedersachsen sind die Nietlöcher ausgerissen. Solche Beschädigungen können entstehen, wenn die Schwertklinge auf ein hartes Ziel geschlagen und geprellt wird. Dabei wird von innen Druck auf die Heftschultern ausgeübt und der dünne Bereich um die Löcher reißt schnell aus. Ebenso zeigen über die komplette Klingenlänge verlaufende Schneiden, dass die gemeinhin als Stichwaffen interpretierten Griffplattenschwerter auch zum Schlagen genutzt werden sollten ${ }^{175}$.

Gebrauchsspuren sind schwierig zu datieren. Es kommen Klingenverletzungen verschiedener Art, Brüche, Nachschärfungen, ausgerissene Nietlöcher und Abnutzung an Griff und Verzierungen in Frage. In Niedersachsen finden sich kleinere oder größere Klingenverletzungen besonders an Griffzungenschwertern. Seltener kommen sie auch an Griffplattenschwertern, nordischen Vollgriffschwertern und Achtkantschwertern vor. Viele Schwerter sind zerbrochen, während Nachschärfungen nur bei wenigen Schwertern zu belegen sind. Dies mag aber auch mit ihrer schwierigen Nachweisbarkeit zusammenhängen, da bei nur geringer Intensität des Nachschleifens die überwiegend starke Korrosion und unsachgemäße Restaurierung in vergangenen Zeiten solche Spuren leicht zunichte gemacht haben können. Bei der Anzahl ausgerissener Nietlöcher stechen besonders die Griffplattenschwerter und „westeuropäischen “ Griffplattenschwerter hervor. Einige Vollgriffschwerter, in erster Linie Achtkantschwerter, zeigen zudem eine Abnutzung im Griffbereich. Insgesamt scheinen die Vollgriffschwerter etwas weniger Gebrauchsspuren aufzuweisen. Verallgemeinerungen dürfen wegen der geringen Zahl an Untersuchungen daraus jedoch nicht gezogen werden, ebenso wenig lässt sich eine Bestätigung für Kristiansens Beobachtung gewinnen, derzufolge Griffzungenschwerter in Dänemark deutlich höhere Gebrauchsspuren aufweisen als Vollgriffschwerter ${ }^{176}$.

Die Seltenheit von Scharten und Klingenverletzungen wird dadurch relativiert, dass ein geübter Kämpfer angesichts des Wertes seiner Waffe und nicht zuletzt zu seinem eigenen Besten einen Kontakt Schneide auf Schneide nach Möglichkeit vermieden haben wird. Daher stellen deutlich erkennbare Schäden an der Schneide einen Ausnahmefall dar ${ }^{177}$. Das Schlagen auf organisches Material führt hinge-

174 Mödlinger 2007, 115-117; dies. 2011, 76.

175 Mödlinger 2007, 115-116; dies. 2011, 75-76.

176 Kristiansen 1984.

177 Molloy 2007, 99; Mödlinger 2007, 115; dies. 2011, 83. 
gen nicht zu spürbaren Schäden ${ }^{178}$. Experimentelle Erfahrungen zeigen zudem, dass kleinere Scharten, die noch den Wulst aufweisen, durch Erhitzen und Dengeln nahezu spurlos beseitigt werden können, so dass scheinbar makellose Schneiden nicht unbedingt ein Zeichen für ein unbenutztes Schwert sind ${ }^{179}$.

Zusammenfassend lässt sich feststellen, dass trotz der Schwierigkeiten bei der Gebrauchsspurenanalyse für die niedersächsischen Schwerter eine praktische Verwendung der Schwerter als Hieb- und Stichwaffen nicht zu leugnen ist. Eine mögliche soziale, symbolische und/oder kultische Funktion beispielsweise als Statussymbol, Herrschaftszeichen oder Opfergabe bleibt davon selbstverständlich unbenommen, ist für die historische Zeit vielfach nachgewiesen und wird insbesondere durch wiederholte Personifizierung von Waffen in jüngeren Epochen verdeutlicht ${ }^{180}$. Ohne dass der bronzezeitliche Mensch das Schwert als Waffe kannte und respektierte, ist seine Rolle als „lesbares“ Symbol jedoch nicht vorstellbar ${ }^{181}$.

\section{Fazit}

Bronzezeitliche Schwerter wurden im Guss hergestellt und anschließend in mehreren Schritten nachbearbeitet. Die Verzierungen können punziert, ziseliert und eventuell graviert sein. Inwieweit feine Ornamente auch bei niedersächsischen Fundstücken mitgegossen wurden, bleibt offen. An zwei nordischen Vollgriffschwertern konnte eine ungewöhnliche Verzierung der Knaufplatte durch eingesetzte Stifte dokumentiert werden, die einen farblichen Kontrast hervorrufen sollte.

Die nordischen Vollgriffschwerter mit ihrem dünnwandigen Guss und die aus dem südlichen Mitteleuropa stammenden Achtkantschwerter mit ihrer dickwandigen Technik wurden sicherlich von den Trägern zweier verschiedener Handwerkstraditionen gefertigt. Diese lassen sich auf ältere Technologiekreise zurückführen und belegen das Fortleben der in der frühen Bronzezeit entwickelten Gusstechnologien in der Mittelbronzezeit. Im Norden verlagern sich die Verbreitungs- und Herstellungszentren vom Aunjetitzer Raum in den Nordischen Kreis. Parallel dazu wird eine Fortführung charakteristischer Aunjetitzer Herstellungstechnologien im Raum des Karpatenbeckens deutlich. Dass beide Zentren noch zu Beginn der Mittelbronzezeit in engem Austausch standen, wurde von vielen Autoren betont und wird u.a. durch den Fund von Dystrup eindrucksvoll belegt. Auch im Süden ist mit der Anknüpfung der Achtkantschwerter an frühbronzezeitliche Gusstechniken ein vergleichbares Bild greifbar.

Nicht nur die Gusstechnik, auch die Schäftung der mittelbronzezeitlichen Vollgriffschwerter lässt Unterschiede

178 Bridgford 2000, Kap. 3.3.

179 Freundl. Mitt. M. Siedlaczek M. A., Berlin.

180 Vgl. Kristiansen 2002, 329-331.

181 Ebenso Mödlinger 2007, 123 Anm. 73. erkennen. So weisen Achtkantschwerter einheitlich eine sogenannte Verkeilschäftung auf, während nordische Vollgriffschwerter der Periode II sehr variantenreich geschäftet sind. In Periode III zeigen alle nordischen Vollgriffschwerter eine gleichartige Schäftung mit Griffangel.

Bemerkenswert ist, dass sich bei nordischen Vollgriffschwertern sehr selten auch Hinweise auf die Adaption der süddeutschen Guss-, vor allem aber Schäftungstechnik finden. Hier deuten sich komplexe Beziehungen und ein offenbar selektiver Technologietransfer zwischen den beiden Handwerkstraditionen an.

Bei allen analysierten Schwertern wurde relativ einheitliche Bronze mit rund 10\% Zinnanteil verwendet. Auch wenn sich keine regelhafte Nutzung verschiedener Legierungen für die unterschiedlichen Schwertteile zeigt, kann das Material durch Dengeln und Zwischenglühen eine hohe Härtesteigerung erzielen. Leider fehlen metallographische Untersuchungen am Fundmaterial zur Kenntnis der genauen Arbeitsschritte. Weiterhin ließ sich nachweisen, dass für die einzelnen Schwertteile mancher Vollgriffschwerter exakt dasselbe Kupfer, aber in anderer Legierung, benutzt wurde. Deswegen und angesichts der teilweise sehr guten Einpassung der Klingen kann man nicht mehr von einer generellen arbeitsteiligen Produktion der Achtkantschwerter in einer Großwerkstatt ausgehen; es müssen eher mehrere Werkstätten in Betracht gezogen werden. Die postulierten Werkstattkreise bei den nordischen Vollgriffschwertern konnten aufgrund der geringen Datenbasis nicht nach technischen Kriterien überprüft werden. Als Herkunftsregion des Kupfers kommt am ehesten der Ostalpenraum, z. B. der Mitterberg, in Frage.

Bei der Distribution von Achtkantschwertern (und ihrer Vorgängerformen) in den Norden, einer lange diskutierten Frage, handelt es sich wohl vornehmlich um Import von süddeutsch verzierten Stücken sowie unverzierten Schwertern, die danach im Norden verziert wurden. Dabei kam es $\mathrm{zu}$ gegenseitigen Kontakten und Beeinflussungen, so dass beispielsweise auch vereinzelt nordisch verzierte Achtkantschwerter im Süden vorkommen.

Die Funktion der Schwerter, deren praktischer Gebrauch lange Zeit aus dem Fokus geraten war und sogar negiert wurde, wird durch das verwendete gut geeignete Material, die fast immer sichere Schäftung und vor allem die Gebrauchsspuren erhellt. Trotz mancher Schwierigkeiten bei der Erhaltung und Datierung fanden sich an jeder Schwertform Niedersachsens zumindest einige Spuren, die ihre Verwendung im Kampf stützen. Neben ihrer primären Funktion als Waffen hatten Schwerter aber sicherlich auch eine soziale und symbolische Bedeutung für den bronzezeitlichen Menschen. 


\section{Literaturverzeichnis}

Aner/Kersten 2008: E. Aner/K. Kersten, Die Funde der älteren Bronzezeit des nordischen Kreises in Dänemark, Schleswig-Holstein und Niedersachsen. Band 12: Viborg Amt (Neumünster 2008).

Ankner 1977: D. Ankner, Röntgenuntersuchungen an Riegseeschwertern. Ein Beitrag zur Typologie. Arch. u. Naturwiss. 1, 1977, 269-459.

Armbruster 2000: B. R. Armbruster, Goldschmiedekunst und Bronzetechnik. Studien zum Metallhandwerk der Atlantischen Bronzezeit auf der Iberischen Halbinsel. Monogr. instrumentum 15 (Montagnac 2000).

Bader 1991: T. Bader, Die Schwerter in Rumänien. PBF IV, 8 (Stuttgart 1991).

Bargel/Schulze 2008: H.-J. Bargel/G. Schulze, Werkstoffkunde ( ${ }^{10}$ Berlin, Heidelberg 2008).

Bartelheim 1998: M. Bartelheim, Studien zur böhmischen Aunjetitzer Kultur. Chronologische und chorologische Untersuchungen. Univforsch. z. Prähist. Arch. 46 (Bonn 1998).

Berger/Pernicka 2010: D. Berger/E. Pernicka, Alles eine Frage des guten Geschmacks - Archäometallurgische Untersuchungen zur künstlichen Korrosion von Buntmetallen in Urin. In: O. Hahn u. a. (Hrsg.), Archäometrie und Denkmalpflege 2010. Jahrestagung vom 15. bis 18. September 2010 in Bochum. Metalla 2010, Sonderh. 3 (Bochum 2010) 82-84.

Bokelmann 1977: K. Bokelmann, Ein Grabhügel der Stein- und Bronzezeit bei Rastorf, Kreis Plön. Offa 34, 1977, 90-99.

Born 1989: H. Born, Antike Lochung in Metall. Acta Praehist. et Arch. 21, 1989, 117-130.

Brandherm/Sicherl 2001: D. Brandherm/B. Sicherl, Überlegungen zur Schwertproduktion der späten Urnenfelderzeit. Bemerkungen zur Herstellung späturnenfelderzeitlicher Vollgriffschwerter anhand zweier Beispiele von nördlich und südlich der Alpen. Arch. Korrbl. 31, 2001, 223-241.

Bridgford 2000: S. D. Bridgford, Weapons, Warfare and Society in Britain 1250-750 BC. (Unpubl. Diss., Sheffield 2000).

Charles 1984: J. A. Charles, The Middle Bronze Age Iron Punch of Southeast Drenthe. Palaeohist. 26, 1984, 95-99.

Christoforidis et al. 1988: A. Christoforidis/E. Pernicka/H. Schickler, Ostalpine Kupferlagerstätten und ihre Bedeutung für die prähistorische Metallgewinnung in Mitteleuropa. Jahrb. RGZM 35 , 1988, 533-536.

Divac/Sedláček 1999: G. Divac/Z. Sedláček (Hrsg.), Hortfund der altbronzezeitlichen Dolche von Praha 6-Suchdol. Fontes Arch. Pragenses Suppl. 1 (Prag 1999).

Drescher 1956/58: H. Drescher, Zur Verwendung von Bronzewerkzeugen in der älteren Bronzezeit. Hammaburg 11, 1956/58, 23-29.

- 1958:-, Der Überfangguss. Ein Beitrag zur vorgeschichtlichen Metalltechnik (Mainz 1958).

- 1961:-, Die Gusstechnik des Vollgriffschwertes aus Meckelfeld, Kr. Harburg. Hammaburg 13, 1961, 57-66.

- 1968: -, Punzen der jüngeren Bronzezeit aus Altmaterial. Jahresschr. Mitteldt. Vorgesch. 52, 1968, 131-142.

- 1978a: -, Ein Vollgriffschwert der Periode II der nordischen Bronzezeit in Hamburger Privatbesitz. Offa 35, 1978, 60-63.

- 1978b: -, Bohrer. I.: Archäologisches. Reallex. Germ. Altkde 3 (Berlin, New York 1978) 189-203.

Driehaus 1961: J. Driehaus, Röntgenuntersuchungen an bronzenen „Vollgriffschwertern“. Germania 39, 1961, 22-31.

- 1968: -, Ein bronzezeitliches Vollgriffschwert aus der Niers. Bonner Jahrb. 168, 1968, 329-369.

Hachmann 1956: R. Hachmann, Süddeutsche Hügelgräber- und Urnenfelderkulturen und ältere Bronzezeit im westlichen Ostseegebiet. Offa $15,1956,43-76$.

- 1957: -, Die frühe Bronzezeit im westlichen Ostseegebiet und ihre mittel- und südosteuropäischen Beziehungen. Chronologische Untersuchungen. Beih. Atlas Urgesch. 6 (Hamburg 1957).
Herner 1989: E. Herner, Spiral Decoration in Early Bronze Age Scandinavia. A Technical and Qualitative Analysis and Study of Production. BAR 552 (Oxford 1989).

Heske et al. 2010: I. Heske/S. Grefen-Peters/M. Posselt/J. Wiethold, Die jungbronzezeitliche Außensiedlung der „Hünenburg“ bei Watenstedt, Lkr. Helmstedt. Vorbericht über die Ausgrabungen 2005-2007. Praehist. Zeitschr. 85, 2010, 159-190.

Hofmann 2003: K. P. Hofmann, Das Achtkantschwert von Alfstedt. Ein Altfund. Arch. Ber. Landkr. Rotenburg (Wümme) 10, 2003, 31-91.

Holste 1953: F. Holste, Die bronzezeitlichen Vollgriffschwerter Bayerns (München 1953).

Hundt 1962: H.-J. Hundt, Zu einigen westeuropäischen Vollgriffschwertern. Jahrb. RGZM 9, 1962, 20-57.

- 1965: -, Produktionsgeschichtliche Untersuchungen über den bronzezeitlichen Schwertguß. Jahrb. RGZM 12, 1965, 41-58.

- 1979: -, Zwei Bronzevollgriffschwerter aus Tirol und Norddeutschland. Arch. Korrbl. 9, 1979, 183-189.

Jantzen 2008: D. Jantzen, Quellen zur Metallverarbeitung im Nordischen Kreis der Bronzezeit. PBF XIX, 2 (Stuttgart 2008).

Joel et al. 1997: E. C. Joel/J. J. Taylor/R. A. Ixer/M. Goodway, Lead Isotope Analysis and the Great Orme Mine. In A. Sinclair/E. Slater/J. Gowlett (Hrsg.), Archaeological Sciences 1995. Oxbow Arch. Monogr. 64 (Oxford 1997) 123-131.

Kaufmann et al. 1996: S. Kaufmann/W. Brockner/D. Klemens/ O. M. Wilbertz, Archäometrische Untersuchungen an bronzezeitlichen Kurzschwertern vom Dahlenburger Typ aus dem nordöstlichen Niedersachsen. Die Kunde N. F. 47, 1996, 263-285.

Kemenczei 1991: T. Kemenczei, Die Schwerter in Ungarn II. PBF IV, 9 (Stuttgart 1991).

Kovács 1994: T. Kovács, Zwei Vollgriffschwerter von Hajdúsámson-Apa-Typ aus dem Donau-Theiss-Zwieschenstromgebiet. Folia Arch. 43, 1994, 51-69.

Krause 2003: R. Krause, Studien zur kupfer- und frühbronzezeitlichen Metallurgie zwischen Karpatenbecken und Ostsee. Vorgesch. Forsch. 24 (Rahden/Westf. 2003).

Kristiansen 1984: K. Kristiansen, Krieger und Häuptlinge in der Bronzezeit Dänemarks. Ein Beitrag zur Geschichte des bronzezeitlichen Schwertes. Jahrb. RGZM 31, 1984, 187-208.

- 2002: -, The Tale of the Sword - Swords and Swordfighters in Bronze Age Europe. Oxford Journal Arch. 21,4, 2002, 319-332.

- /Larsson 2005: - /T. B. Larsson, The Rise of Bronze Age Society. Travels, Transmissions and Transformations (Cambridge 2005).

Laux 2009: F. Laux, Die Schwerter in Niedersachsen. PBF IV, 17 (Stuttgart 2009).

- 2011: -, Die Dolche in Niedersachsen. PBF VI, 13 (Stuttgart 2011).

Lemonnier 1993: P. Lemonnier, Introduction. In: P. Lemonnier (Hrsg.), Technological Choice. Transformation in Material Cultures since the Neolithic (London, New York 1993) 1-35.

Meller 2002: H. Meller, Die Himmelsscheibe von Nebra - ein frühbronzezeitlicher Fund von außergewöhnlicher Bedeutung. Arch. Sachsen-Anhalt 1, 2002, 7-31.

Mödlinger 2007: M. Mödlinger, Herstellung und Qualität mittelund spätbronzezeitlicher Schwerter aus Österreich. Das Altertum 52, 2007, 101-130.

- 2008:-, Micro-X-ray Computer Tomography in Archaeology: Analyses of a Bronze Age Sword. Insight 50,6, 2008, 323-326.

- 2011:-, Herstellung und Verwendung bronzezeitlicher Schwerter Mitteleuropas. Eine vertiefende Studie zur mittelbronze- und urnenfelderzeitlichen Bewaffnung und Sozialstruktur. Univforsch. Prähist. Arch. 193 (Bonn 2011).

Molloy 2007: B. P. C. Molloy, What's the Bloody Point? Bronze Age Swordmanship in Ireland and Britain. In: B. P. C. Molloy (Hrsg.), The Cutting Edge. Studies in Ancient and Medieval Combat (Stroud 2007) 90-111. 
Neipert 2006: M. Neipert, Der „Wanderhandwerker“. Archäologisch-ethnographische Untersuchungen. Tübinger Texte 6 (Rahden/Westf. 2006).

Oldeberg 1976: A. Oldeberg, Die ältere Metallzeit in Schweden II (Stockholm 1976).

Ottenjann 1969: H. Ottenjann, Die nordischen Vollgriffschwerter der älteren und mittleren Bronzezeit. Röm.-Germ. Forsch. 30 (Berlin 1969).

Pernicka 1990: E. Pernicka, Gewinnung und Verbreitung der Metalle in prähistorischer Zeit. Jahrb. RGZM 37,1, 1990, 21-129.

- et al. 2008: -/C.-H. Wunderlich/A. Reichenberger/H. Meller/G. Borg, Zur Echtheit der Himmelsscheibe von Nebra - eine kurze Zusammenfassung der durchgeführten Untersuchungen. Arch. Korrbl. 38, 2008, 331-352.

von Quillfeldt 1995: I. von Quillfeldt, Die Vollgriffschwerter in Süddeutschland. PBF IV, 11 (Stuttgart 1995).

Randsborg 1968: K. Randsborg, Von Periode II zu III. Chronologische Studien über die ältere Bronzezeit Südskandinaviens und Norddeutschlands. Acta Arch. (København) 39, 1968, 1-142.

Rasmussen/Boas 2006: L. W. Rasmussen/N. A. Boas, The Dystrup Swords: A Hoard with Eight Short Swords from the Early Bronze Age. Journal Danish Arch. 14, 2006, 87-108.

Riederer 2004: Ergebnisse metallanalytischer Untersuchungen von Bronzeschwertern. In: H. Wüstemann, Die Schwerter in Ostdeutschland. PBF IV, 15 (Stuttgart 2004) 259-329.

Rohl/Needham 1998: B. Rohl/S. Needham, The Circulation of Metal in the British Bronze Age: The Application of Lead Isotope Analysis. British Museum Occasional Papers 102 (London 1998).

Rønne 1989: P. Rønne, Early Bronze Age Spiral Ornament - the Technical Background. Journal Danish Arch. 8, 1989, 126-143.

Schmidt 2009: J.-P. Schmidt, Ein jungbronzezeitliches Griffzungenschwert vom Typ Mainz aus Radepohl, Lkr. Parchim. Arch. Ber. Mecklenburg-Vorpommern 16, 2009, 28-32.

Schwab et al. 2007: R. Schwab/C.-H. Wunderlich/K. Peisker, Feine Linien in Bronze - Ein Beitrag zur Metallbearbeitungstechnik der Bronzezeit. Jahresschr. Mitteldt. Vorgesch. 91, 2007, 243-259. Schwenzer 2004a: S. Schwenzer, Frühbronzezeitliche Vollgriffdolche. Typologische, chronologische und technische Studien auf der Grundlage einer Materialaufnahme von Hans-Jürgen Hundt. Kat. vor- u. frühgesch. Altertümer 36 (Mainz 2004).
- 2004b: -, Zum Einfluß der Aunjetitzer Kultur auf die frühbronzezeitliche Metallverarbeitung in Mitteleuropa. Arch. Korrbl. 34, 2004, 193-210.

- 2009a: -, Vollgriffdolche der frühen Bronzezeit in der Schweiz Statussymbole und Zeugnisse gusstechnischen Könnens. Helvetia Arch. 40, 2009, 66-96.

- 2009b: -, Kommunikationskonzepte in der prähistorischen Archäologie. In: A. Krenn-Leeb u. a. (Hrsg), Mobilität, Migration und Kommunikation in Europa während des Neolithikums und der Bronzezeit. Beiträge der Sitzungen der AGs Neolithikum und Bronzezeit, Xanten 2006. Beitr. Ur-Frühgesch. Mitteleuropas 53 = Varia neolithica V (Langenweissbach 2009) 7-19.

Sicherl 1996: B. Sicherl, Nochmals zu den ältesten Schwertformen Niedersachsens. Die Kunde N. F. 47, 1996, 287-302.

Thrane 2006: H. Thrane, Swords and other Weapons in the Nordic Bronze Age: Technology, Treatment and Context. In: T. Otto/ H. Thrane/H. Vandkilde (Hrsg.), Warfare and Society. Archaeological and Social Anthropological Perspectives (Aarhus 2006) 491-504.

Vandkilde 2006: H. Vandkilde, Archaeology and War: Presentations of Warriors and Peasants in Archaeological Interpretations. In: T. Otto/H. Thrane/H. Vandkilde (Hrsg.), Warfare and Society. Archaeological and Social Anthropological Perspectives (Aarhus 2006) 57-73.

Willroth 1999: K.-H. Willroth, Krieger, Häuptlinge oder „nur“ freie Bauern. Zum Wandel in der Bronzezeitforschung. In: W. Budesheim/H. Keiling (Hrsg.), Zur Bronzezeit in Norddeutschland. Beiträge für Wissenschaft und Kultur 3. Seminar in Schwarzenbek am 16. Sept. 1994 (Neumünster 1999) 39-66.

Wüstemann 1999: H. Wüstemann, Zu einigen Ergebnissen radiographischer Untersuchungen an bronzenen Vollgriffdolchen und Vollgriffschwertern. In: W. Budesheim/H. Keiling (Hrsg.), Zur Bronzezeit in Norddeutschland. Beiträge für Wissenschaft und Kultur 3. Seminar in Schwarzenbek am 16. Sept. 1994 (Neumünster 1999) 123-144.

- 2004: -, Die Schwerter in Ostdeutschland. PBF IV, 15 (Stuttgart 2004).

Zich 1996: B. Zich, Studien zur regionalen und chronologischen Gliederung der nördlichen Aunjetitzer Kultur. Vorgesch. Forsch. 20 (Berlin, New York 1996).

Jan-Heinrich Bunnefeld M.A., Georg-August-Universität Göttingen, Seminar für Ur- und Frühgeschichte, Nikolausberger Weg 15, 37073 Göttingen. E-Mail: jan-heinrich.bunnefeld@gmx.de

Dr. Stefan Schwenzer, Tile-Wardenberg-Str. 28, 10555 Berlin.E-Mail: schwenzer@bronzezeit-online.de 


\section{Anhang: Katalog}

\section{Griffplattenschwerter}

Kat.-Nr.: 1

Fundort: Baven, Gde. Hermannsburg, Lkr. Celle

Typ u. Variante: Typ Wohlde, Variante Baven

Datierung: Periode I b

Museum u. Inv.-Nr.: LM Hannover, 426/32

Literatur: Laux 2009, 31 Nr. 45

Kat.-Nr.: 2

Fundort: Baven, Gde. Hermannsburg, Lkr. Celle

Typ u. Variante: Typ Sögel, Variante Bargloy

Datierung: Periode I b

Museum u. Inv.-Nr.: LM Hannover, 425/32

Literatur: Laux 2009, 26 Nr. 32

Kat.-Nr.: 3

Fundort: Baven, Gde. Hermannsburg, Lkr. Celle

Typ u. Variante: Typ Baven, Variante Baven

Datierung: Periode I b

Museum u. Inv.-Nr.: LM Hannover, 430/32

Literatur: Laux 2009, 39 Nr. 66

Kat.-Nr.: 4

Fundort: Bokeloh, Stadt Wunstorf, Lkr. Hannover

Typ u. Variante: Typ Sögel, Variante Bockel

Datierung: Periode I b

Museum u. Inv.-Nr.: LM Hannover, 16158

Literatur: Laux 2009, 22 Nr. 17

Kat.-Nr.: 5

Fundort: Buxtehude-Altkloster, Lkr. Stade

Typ u. Variante: Typ Harburg, Variante Ehestorf

Datierung: Periode I b-Periode II

Museum u. Inv.-Nr.: LM Hannover, 5436a/b

Literatur: Laux 2009, 40 Nr. 69

Kat.-Nr.: 6

Fundort: Ehestorf, Gde. Elstorf, Lkr. Rotenburg (Wümme)

Typ u. Variante: Typ Harburg, Variante Ehestorf

Datierung: Periode I b-Periode II

Museum u. Inv.-Nr.: LM Hannover, 5522

Literatur: Laux 2009, 40 Nr. 71

\section{Kat.-Nr.: 7}

Fundort: Garlstorf, Lkr. Harburg

Typ u. Variante: Griffplattenschwert von „westeuropäischem“ Typ, Langschwert/Rapier, Variante Garlstorf

Datierung: Periode II

Museum u. Inv.-Nr.: LM Hannover, 4704

Literatur: Laux 2009, 46 Nr. 87

Kat.-Nr.: 8

Fundort: Granstedt, Gde. Selsingen, Lkr. Rotenburg (Wümme)

Typ u. Variante: Typ Wohlde, Variante Baven

Datierung: Periode I b

Museum u. Inv.-Nr.: Mus. Bremervörde, Slg. König 81

Literatur: Laux 2009, 31 Nr. 46

Kat.-Nr.: 9

Fundort: Granstedt, Gde. Selsingen, Lkr. Rotenburg (Wümme)

Typ u. Variante: Griffplattenschwert von „westeuropäischem“ Typ, Langschwert/Rapier, Variante Granstedt

Datierung: Periode II

Museum u. Inv.-Nr.: Mus. Bremervörde, Slg. König 80

Literatur: Laux 2009, 48 Nr. 98
Kat.-Nr.: 10

Fundort: Hambostel, Stadt Soltau, Lkr. Soltau-Fallingbostel Typ u. Variante: Zwischenform der Typen Sögel und Wohlde Datierung: Periode I b

Museum u. Inv.-Nr.: LM Hannover, 8267

Literatur: Laux 2009, 35 Nr. 58

Kat.-Nr.: 11

Fundort: Heine, Gde. Wulsbüttel, Lkr. Cuxhaven

Typ u. Variante: Typ Sögel, Variante Grapperhausen

Datierung: Periode I b

Museum u. Inv.-Nr.: Mus. Bad Bederkesa, IV/20/67

Literatur: Laux 2009, 25 Nr. 28

Kat.-Nr.: 12

Fundort: Helmste, Gde. Deinste, Lkr. Stade

Typ u. Variante: Typ Sögel, Variante Bockel

Datierung: Periode I b

Museum u. Inv.-Nr.: Mus. Stade, 2318

Literatur: Laux 2009, 22 Nr. 16

Kat.-Nr.: 13

Fundort: Klein Henstedt, Gde. Prinzhoefte, Lkr. Oldenburg

Typ u. Variante: Zwischenform der Typen Sögel und Wohlde

Datierung: Periode I b

Museum u. Inv.-Nr.: Mus. Nienburg, 140

Literatur: Laux 2009, 35 Nr. 57

Kat.-Nr.: 14

Fundort: Lemförde, Lkr. Diepholz

Typ u. Variante: Typ Baven, Variante Stolzenau

Datierung: Periode I b

Museum u. Inv.-Nr.: LM Hannover, 5768

Literatur: Laux 2009, 38 Nr. 63

Kat.-Nr.: 15

Fundort: Mehlbergen, Gde. Balge, Lkr. Nienburg

Typ u. Variante: Typ Wohlde, Variante Mehlbergen

Datierung: Periode I b

Museum u. Inv.-Nr.: Mus. Nienburg, 141

Literatur: Laux 2009, 32 Nr. 50

Kat.-Nr.: 16

Fundort: Mellinghausen, Lkr. Diepholz

Typ u. Variante: Typ Sögel, Variante Bockel

Datierung: Periode I b

Museum u. Inv.-Nr.: Mus. Nienburg, U. 142

Literatur: Laux 2009, 21-22 Nr. 12

Kat.-Nr.: 17

Fundort: Nienburg, Lkr. Nienburg

Typ u. Variante: Typ Wohlde, Variante Mehlbergen

Datierung: Periode I b

Museum u. Inv.-Nr.: LM Hannover, 11014

Literatur: Laux 2009, 32 Nr. 49

Kat.-Nr.: 18

Fundort: Ostendorf, Gde. Bremervörde, Lkr. Rotenburg (Wümme)

Typ u. Variante: Typ Baven

Datierung: Periode I b

Museum u. Inv.-Nr.: Mus. Bremervörde, o. Nr.

Literatur: unpubliziert 
Kat.-Nr.: 19

Fundort: Ostereistedt, Lkr. Rotenburg (Wümme)

Typ u. Variante: Typ Harburg, Variante Stübeckshorn

Datierung: Periode I b

Museum u. Inv.-Nr.: LM Hannover, 28285

Literatur: Laux 2009, 41 Nr. 78

Kat.-Nr.: 20

Fundort: Osterholz-Scharmbeck, Lkr. Osterholz

Typ u. Variante: Typ Harburg, Variante Stübeckshorn

Datierung: Periode I b

Museum u. Inv.-Nr.: Mus. Stade, 432

Literatur: Laux 2009, 41 Nr. 76

Kat.-Nr.: 21

Fundort: Ottensen, Stadt Buxtehude, Lkr. Stade

Typ u. Variante: Griffplattenschwert mit fünf Pflocknieten, Variante Agathenburg

Datierung: Periode II

Museum u. Inv.-Nr.: Mus. Stade, 2986

Literatur: Laux 2009, 64 Nr. 151

Kat.-Nr.: 22

Fundort: Soderstorf, Lkr. Lüneburg

Typ u. Variante: Griffplattenschwert von „westeuropäischem“ Typ, Langschwert/Rapier, Variante Garlstorf

Datierung: Periode II

Museum u. Inv.-Nr.: LM Hannover, 4707

Literatur: Laux 2009, 46 Nr. 88

Kat.-Nr.: 23

Fundort: Soltau, Lkr. Soltau-Fallingbostel

Typ u. Variante: Typ Baven, Variante Baven

Datierung: Periode I b

Museum u. Inv.-Nr.: LM Hannover, 366/52

Literatur: Laux 2009, 39 Nr. 64

Kat.-Nr.: 24

Fundort: Soltau, Lkr. Soltau-Fallingbostel

Typ u. Variante: Griffplattenschwert mit fünf Pflocknieten

Datierung: Periode III

Museum u. Inv.-Nr.: LM Hannover, 4698

Literatur: Laux 2009, 63 Nr. 143

Kat.-Nr.: 25

Fundort: Umgebung von Soltau, Lkr. Soltau-Fallingbostel

Typ u. Variante: Typ Harburg, Variante Ehestorf

Datierung: Periode I b-Periode II

Museum u. Inv.-Nr.: LM Hannover, 14282

Literatur: Laux 2009, 41 Nr. 74

Kat.-Nr.: 26

Fundort: Steinkirchen, Lkr. Stade

Typ u. Variante: Typ Wohlde

Datierung: Periode I b

Museum u. Inv.-Nr.: Mus. Stade, 726

Literatur: Laux 2009, 32 Nr. 52

Kat.-Nr.: 27

Fundort: Umgebung von Bremen

Typ u. Variante: Typ Wohlde, Variante Grethem

Datierung: Periode I b

Museum u. Inv.-Nr.: Mus. Bremen, V. 308

Literatur: Laux 2009, 33 Nr. 54
Kat.-Nr.: 28

Fundort: Wessenstedt, Gde. Natendorf, Lkr. Uelzen

Typ u. Variante: Griffplattenschwert von „,westeuropäischem“ Typ, Langschwert/Rapier, Variante Granstedt

Datierung: Periode II

Museum u. Inv.-Nr.: LM Hannover, 14255

Literatur: Laux 2009, 48 Nr. 100

\section{Griffzungenschwerter}

Kat.-Nr.: 29

Fundort: Bostelwiebeck, Gde. Altenmedingen, Lkr. Uelzen Typ u. Variante: Typ Sprockhoff I a, Variante Bostelwiebek Datierung: Periode III

Museum u. Inv.-Nr.: LM Hannover, 4697

Literatur: Laux 2009, 102 Nr. 253

Kat.-Nr.: 30

Fundort: Bramstedt, Lkr. Cuxhaven

Typ u. Variante: Typ Sprockhoff I a, Variante Frelsdorf

Datierung: Periode II

Museum u. Inv.-Nr.: LM Hannover, 5441

Literatur: Laux 2009, 100 Nr. 242

Kat.-Nr.: 31

Fundort: Eggestedt, Gde. Schwanewede, Lkr. Osterholz

Typ u. Variante: Typ Nenzingen, Variante Dahlenburg

Datierung: Periode III

Museum u. Inv.-Nr.: LM Hannover, 5438

Literatur: Laux 2009, 111 Nr. 279

Kat.-Nr.: 32

Fundort: Farven, Lkr. Rotenburg (Wümme)

Typ u. Variante: Typ Sprockhoff I a

Datierung: Periode II

Museum u. Inv.-Nr.: Mus. Stade, 2962a

Literatur: Laux 2009, 104 Nr. 259

Kat.-Nr.: 33

Fundort: Gollern, Lkr. Uelzen

Typ u. Variante: Typ Schweringen, Variante Schweringen

Datierung: Periode III

Museum u. Inv.-Nr.: LM Hannover, 13084

Literatur: Laux 2009, 123 Nr. 324

Kat.-Nr.: 34

Fundort: Harsefeld, Lkr. Stade

Typ u. Variante: Typ Sprockhoff I a, Variante Westerwanna

Datierung: Periode II

Museum u. Inv.-Nr.: LM Hannover, 5440a-c

Literatur: Laux 2009, 101 Nr. 246

Kat.-Nr.: 35

Fundort: Langen, Lkr. Cuxhaven

Typ u. Variante: Typ Sprockhoff I b

Datierung: Periode II

Museum u. Inv.-Nr.: Mus. Stade, 244

Literatur: Laux 2009, 106 Nr. 263

Kat.-Nr.: 36

Fundort: Nindorf, Gde. Lamstedt, Lkr. Cuxhaven

Typ u. Variante: Typ Sprockhoff I b, Variante Nindorf

Datierung: Periode II

Museum u. Inv.-Nr.: LM Hannover, 5377

Literatur: Laux 2009, 109 Nr. 269 
Kat.-Nr.: 37

Fundort: Ruschwedel, Flecken Harsefeld, Lkr. Stade

Typ u. Variante: Typ Sprockhoff I b, Variante Ruschwedel

Datierung: Periode II

Museum u. Inv.-Nr.: Mus. Stade, 3730

Literatur: Laux 2009, 105 Nr. 261

Kat.-Nr.: 38

Fundort: Sternberg, Lkr. Stade

Typ u. Variante: Typ Sprockhoff I a, Variante Westerwanna

Datierung: Periode II

Museum u. Inv.-Nr.: Mus. Stade, 430.247

Literatur: Laux 2009, 101 Nr. 251

Kat.-Nr.: 39

Fundort: Umgebung von Stade, Lkr. Stade

Typ u. Variante: Typ Sprockhoff I a, Variante Westerwanna Datierung: Periode II

Museum u. Inv.-Nr.: Mus. Stade, 42

Literatur: Laux 2009, 101 Nr. 250

Kat.-Nr.: 40

Fundort: Wehden, Gde. Schiffdorf, Lkr. Cuxhaven

Typ u. Variante: Typ Sprockhoff I a, Variante Westerwanna

Datierung: Periode II

Museum u. Inv.-Nr.: LM Hannover, 15552 a-d

Literatur: Laux 2009, 101 Nr. 244

\section{Nordische Vollgriffschwerter/-dolche}

Kat.-Nr.: 41

Fundort: Apensen, Lkr. Stade

Typ u. Variante: Nordisches Vollgriffschwert, Typ B1

Datierung: Periode II

Museum u. Inv.-Nr.: ALM Schleswig, Slg. Roselius 167

Literatur: Ottenjann 1969, 86 Nr. 75; Laux 2009, 82 Nr. 190

Kat.-Nr.: 42

Fundort: Apensen, Lkr. Stade

Typ u. Variante: Nordisches Vollgriffschwert, Typ K3

Datierung: Periode II

Museum u. Inv.-Nr.: ALM Schleswig, Slg. Roselius 169

Literatur: Laux 2009, 79 Nr. 185

Kat.-Nr.: 43

Fundort: Deutsch-Evern, Lkr. Lüneburg

Typ u. Variante: Nordisches Vollgriffschwert (Per. III), Typ F2 oder G2

Datierung: Periode III

Museum u. Inv.-Nr.: LM Hannover, 4654

Literatur: Laux 2009, 87 Nr. 200

Kat.-Nr.: 44

Fundort: Estorf, Lkr. Stade

Typ u. Variante: Nordisches Vollgriffschwert, Typ E1

Datierung: Periode II

Museum u. Inv.-Nr.: Mus. Stade, 725

Literatur: Ottenjann 1969, 90 Nr. 148; Laux 2009, 78 Nr. 183

Kat.-Nr.: 45

Fundort: Estorf, Lkr. Stade

Typ u. Variante: Nordisches Vollgriffschwert, Klingenbruchstück

Datierung: Periode II

Museum u. Inv.-Nr.: Mus. Stade, 725

Literatur: Ottenjann 1969, 90 Nr. 148; Laux 2009, 78 Nr. 183
Kat.-Nr.: 46

Fundort: Hammah, Lkr. Stade

Typ u. Variante: Nordischer Vollgriffdolch, Typ A1

Datierung: Periode II

Museum u. Inv.-Nr.: LM Hannover, 17024

Literatur: Ottenjann 1969, 83 Nr. 34; Laux 2011, 18 Nr. 10

Kat.-Nr.: 47

Fundort: Harsefeld, Lkr. Stade

Typ u. Variante: Nordisches Vollgriffschwert, Typ E2

Datierung: Periode II

Museum u. Inv.-Nr.: Mus. Stade, 807

Literatur: Ottenjann 1969, 90 Nr. 137; Laux 2009, 79 Nr. 184

Kat.-Nr.: 48

Fundort: Loxstedt, Lkr. Cuxhaven

Typ u. Variante: Nordisches Vollgriffschwert, Typ E2

Datierung: Periode II

Museum u. Inv.-Nr.: LM Hannover, 7268

Literatur: Ottenjann 1969, 91 Nr. 164; Laux 2009, 85 Nr. 197

Kat.-Nr.: 49

Fundort: Meckelfeld, Gde. Seevetal, Lkr. Harburg

Typ u. Variante: Nordisches Vollgriffschwert (Per. III), Typ A1

Datierung: Periode III

Museum u. Inv.-Nr.: Helms-Museum Hamburg, V. 56:179

Literatur: Drescher 1961; Laux 2009, 85-86 Nr. 198

Kat.-Nr.: 50

Fundort: Nordholz, Lkr. Cuxhaven

Typ u. Variante: Nordisches Vollgriffschwert, Typ B4

Datierung: Periode II

Museum u. Inv.-Nr.: Mus. Vegesack, V.161

Literatur: Laux 2009, 83 Nr. 191

Kat.-Nr.: 51

Fundort: Umgebung von Stade

Typ u. Variante: Nordisches Vollgriffschwert, Typ M4

Datierung: Periode II

Museum u. Inv.-Nr.: Mus. Stade, 45

Literatur: Ottenjann 1969, 100 Nr. 313; Laux 2009, 81 Nr. 188

Kat.-Nr.: 52

Fundort: Umgebung von Stade

Typ u. Variante: Nordisches Vollgriffschwert, Typ A1

Datierung: Periode II

Museum u. Inv.-Nr.: Mus. Stade, 276

Literatur: Hundt 1962, 35-36; Ottenjann 1969, 83 Nr. 29; Laux 2009, 85 Nr. 196

Kat.-Nr.: 53

Fundort: Westerwanna, Gde. Wanna, Lkr. Cuxhaven

Typ u. Variante: Nordisches Vollgriffschwert, Typ K3

Datierung: Periode II

Museum u. Inv.-Nr.: LM Hannover, 5431

Literatur: Ottenjann 1969, 96 Nr. 249; Laux 2009, 80 Nr. 186

Kat.-Nr.: 54

Fundort: Wiepenkathen, Stadt Stade, Lkr. Stade

Typ u. Variante: Nordisches Vollgriffschwert, Typ K2

Datierung: Periode II

Museum u. Inv.-Nr.: Mus. Stade, 238

Literatur: Ottenjann 1969, 95 Nr. 224; Laux 2009, 77 Nr. 182 


\section{Achtkantschwerter}

Kat.-Nr.: 55

Fundort: Bremervörde, Lkr. Rotenburg (Wümme)

Typ u. Variante: Variante Ottensen/Typ Vasby

Datierung: Periode II

Museum u. Inv.-Nr.: LM Hannover, 5425

Literatur: Laux 2009, 73 Nr. 172

Kat.-Nr.: 56

Fundort: Fundort unbekannt

Typ u. Variante: Variante Ottensen/Typ Vasby

Datierung: Periode II

Museum u. Inv.-Nr.: LM Hannover, 6282

Literatur: Laux 2009, 73 Nr. 174

Kat.-Nr.: 57

Fundort: Kuhla, Gde. Himmelpforten, Lkr. Stade

Typ u. Variante: Variante Himmelpforten/Typ Hausmoning

Datierung: Periode II

Museum u. Inv.-Nr.: Mus. Stade, 4245

Literatur: Laux 2009, 74 Nr. 178

Kat.-Nr.: 58

Fundort: Lühnenspecken, Gde. Fredenbeck, Lkr. Stade

Typ u. Variante: Variante Lühnenspecken/Typ „Schonen“ bzw. Klakring

Datierung: Periode II

Museum u. Inv.-Nr.: Mus. Stade, 233

Literatur: Laux 2009, 72 Nr. 169

Kat.-Nr.: 59

Fundort: Meckelstedt, Gde. Lintig, Lkr. Cuxhaven

Typ u. Variante: Vorgängerform Achtkantschwert

Datierung: Periode II

Museum u. Inv.-Nr.: LM Hannover, 1555

Literatur: Laux 2009, 71 Nr. 168

Kat.-Nr.: 60

Fundort: Ober Ochtenhausen, Gde. Sandbostel, Lkr. Rotenburg (Wümme)

Typ u. Variante: -

Datierung: Periode II

Museum u. Inv.-Nr.: Mus. Bremerhaven, V/349/61 bzw. 92.1669

Literatur: Laux 2009, 75 Nr. 180

Kat.-Nr.: 61

Fundort: Ottensen, Stadt Buxtehude, Lkr. Stade

Typ u. Variante: Variante Ottensen/Typ Vasby

Datierung: Periode II

Museum u. Inv.-Nr.: Mus. Stade, 4819

Literatur: Laux 2009, 73 Nr. 171

Kat.-Nr.: 62

Fundort: Schwinge, Gde. Fredenbeck, Lkr. Stade

Typ u. Variante: Variante Lühnenspecken/Typ „Schonen“ bzw. Klakring
Datierung: Periode II

Museum u. Inv.-Nr.: Mus. Stade, 3938

Literatur: Laux 2009, 72 Nr. 170

Kat.-Nr.: 63

Fundort: Umgebung von Stade

Typ u. Variante: Variante Himmelpforten/Typ Hausmoning

Datierung: Periode II

Museum u. Inv.-Nr.: Mus. Stade, 44

Literatur: Laux 2009, 74 Nr. 179

Kat.-Nr.: 64

Fundort: Wedel, Gde. Fredenbeck, Lkr. Stade

Typ u. Variante: Vorgängerform Achtkantschwert

Datierung: Periode II

Museum u. Inv.-Nr.: Mus. Stade, 603

Literatur: Hundt 1979; Laux 2009, 70 Nr. 167

Kat.-Nr.: 65

Fundort: Wiepenkathen, Stadt Stade, Lkr. Stade

Typ u. Variante: Typ Erbach, Variante Roskilde, Sonderform

Datierung: Periode II

Museum u. Inv.-Nr.: Mus. Stade, 2287a

Literatur: Laux 2009, 76 Nr. 181

\section{Sonstige Fundstücke}

Kat.-Nr.: 66

Fundort: Hambostel, Stadt Soltau, Lkr. Soltau-Fallingbostel

Typ u. Variante: Vollgriffdolch, Variante Gandersheim

Datierung: Periode I b-Periode II

Museum u. Inv.-Nr.: LM Hannover, 8269

Literatur: Laux 1971, 227-228 Nr. 342; Laux 2011, 13 Nr. 4

Kat.-Nr.: 67

Fundort: Immenbeck, Stadt Buxtehude, Lkr. Stade

Typ u. Variante: Schwertknauf

Datierung: Periode I b-Periode II

Museum u. Inv.-Nr.: Mus. Bremen, 8010

Literatur: Laux 2009, 43 Nr. 82

Kat.-Nr.: 68

Fundort: Ruschwedel (im Mus. Stade unter „Kammerbusch“), Flecken Harsefeld, Lkr. Stade

Typ u. Variante: Vollgriffschwert

Datierung: Periode I b-Periode II

Museum u. Inv.-Nr.: Mus. Stade, 4370

Literatur: Laux 2009, 15 Nr. 2

Kat.-Nr.: 69

Fundort: Umgebung von Stade, Lkr. Stade

Typ u. Variante: Vollgriffdolch, Variante Wohlde

Datierung: Periode I b-Periode II

Museum u. Inv.-Nr.: Mus. Stade, 872

Literatur: Hachmann 1957, 201 Nr. 329; Laux 2011, 13 Nr. 2 


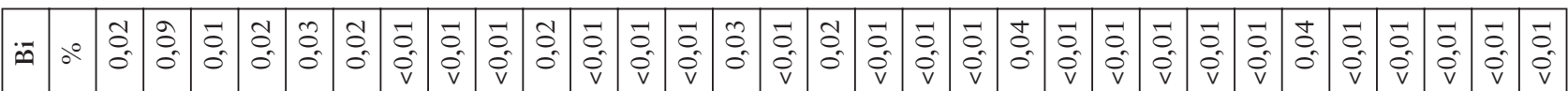

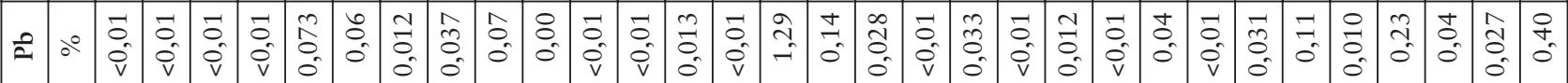

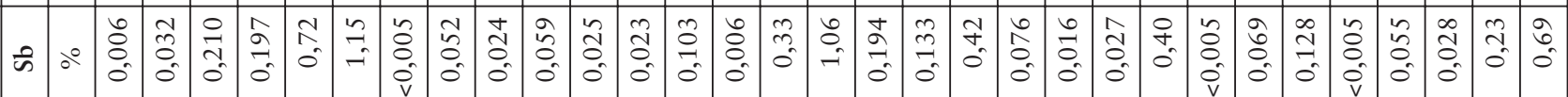

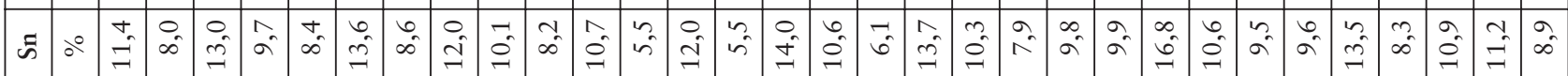

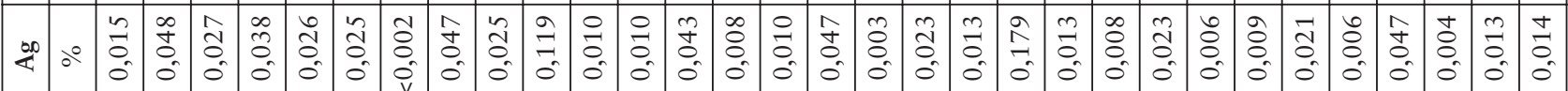

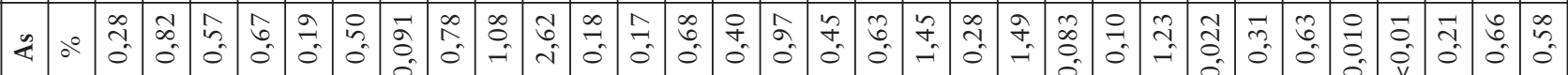

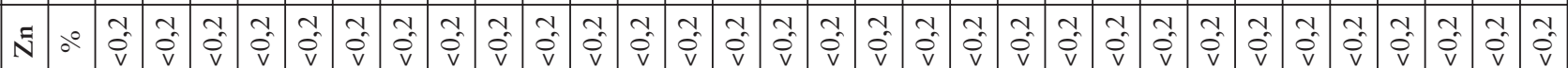

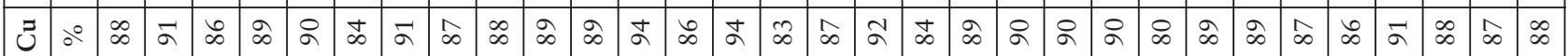

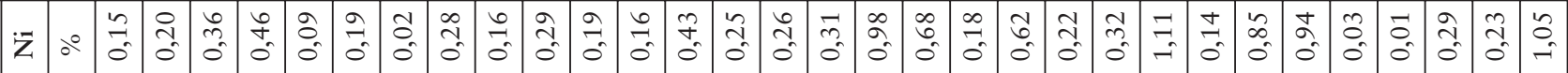

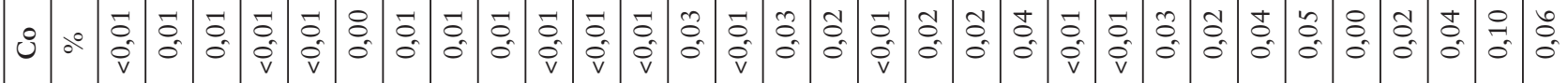

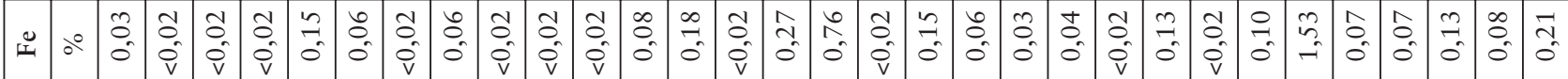

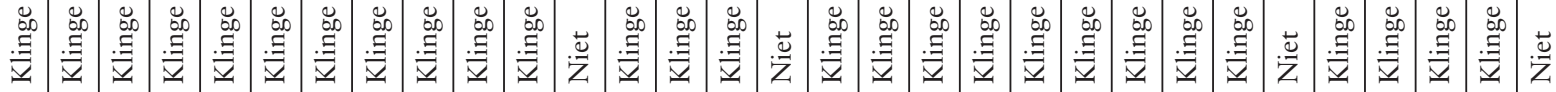

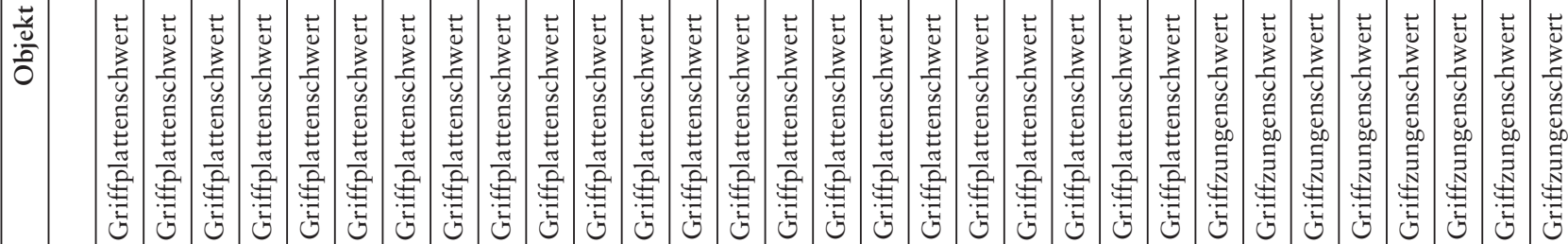

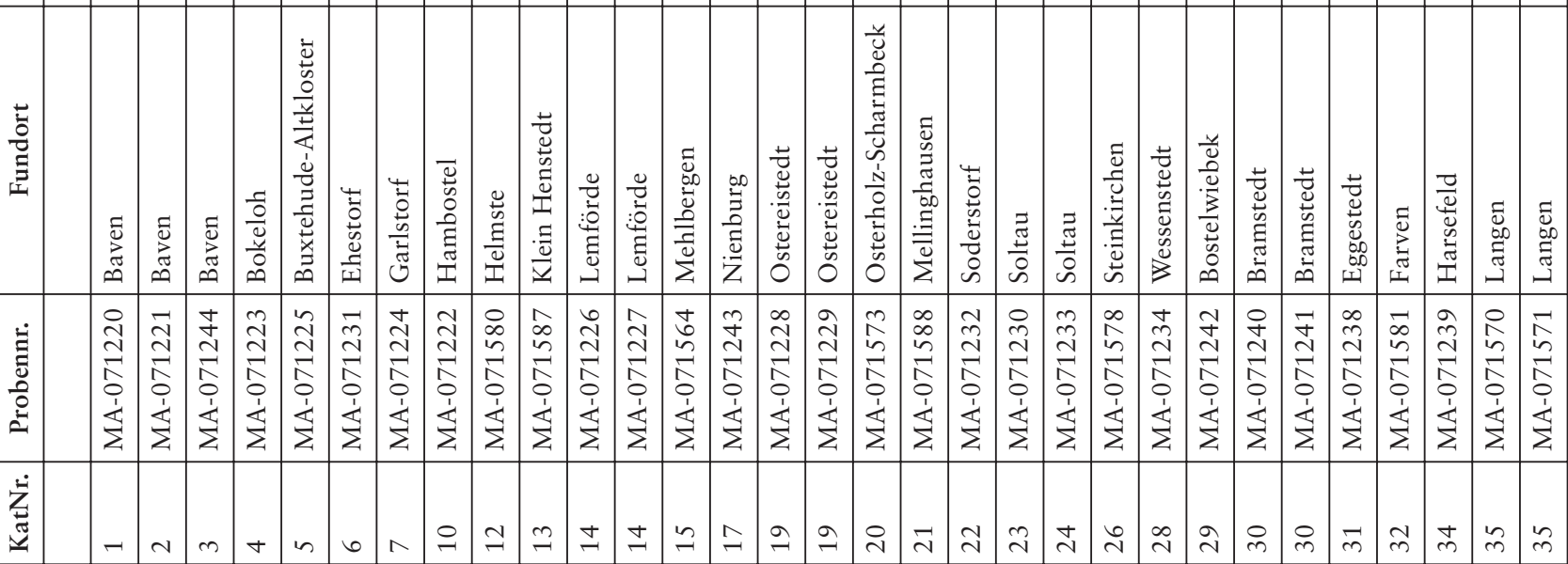




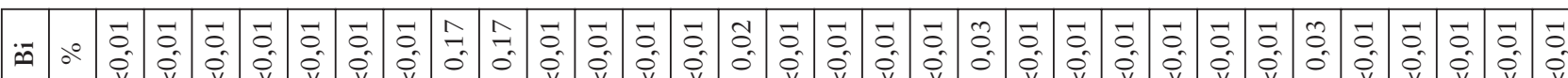

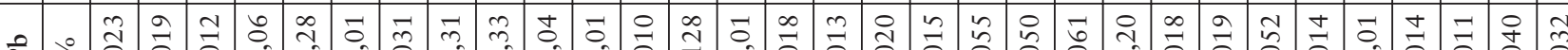

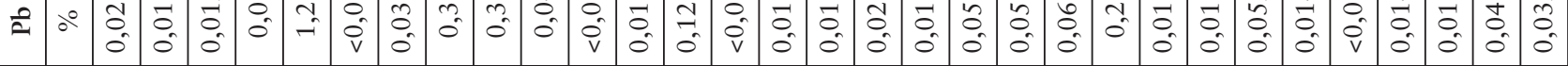

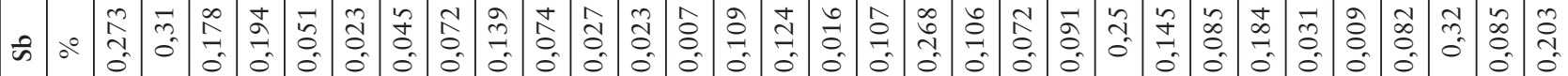

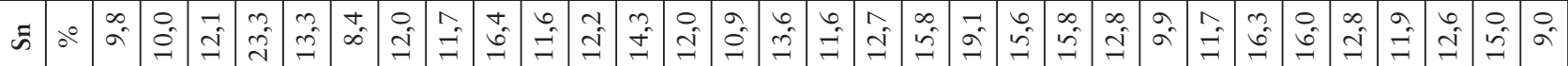
L

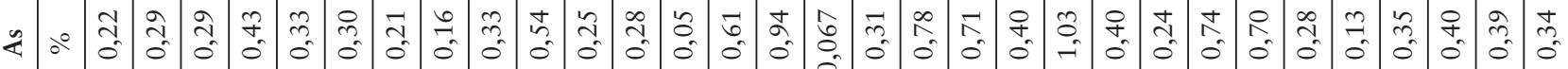

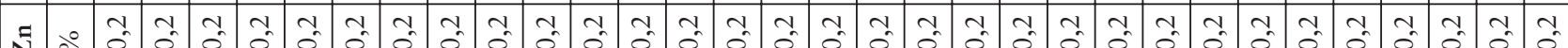

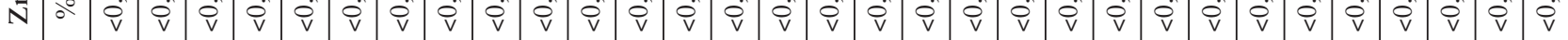

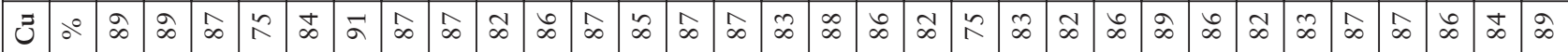

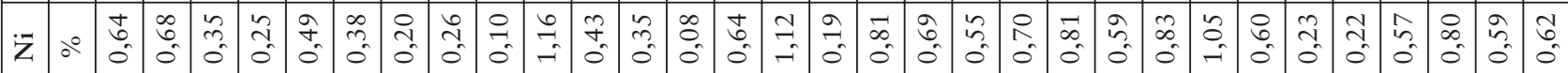

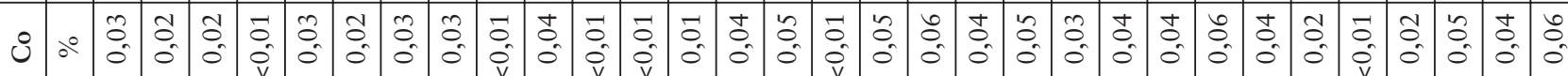

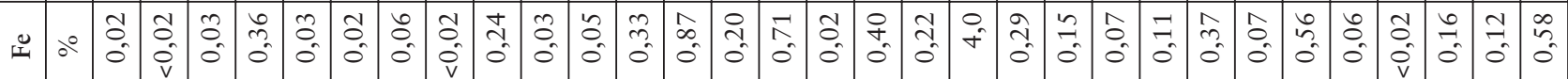

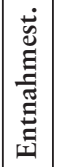

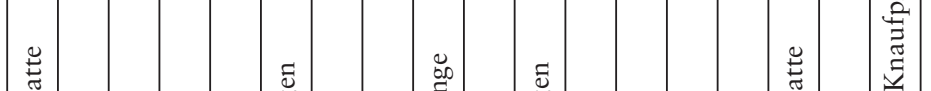

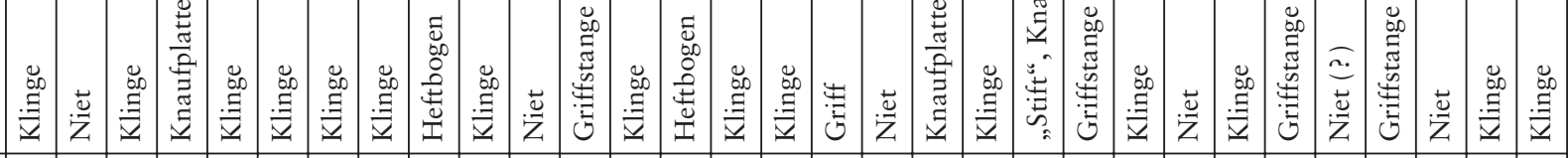
芯 $\quad \begin{aligned} & : \\ & 0\end{aligned}$

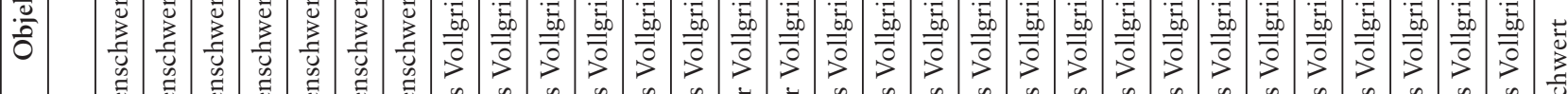

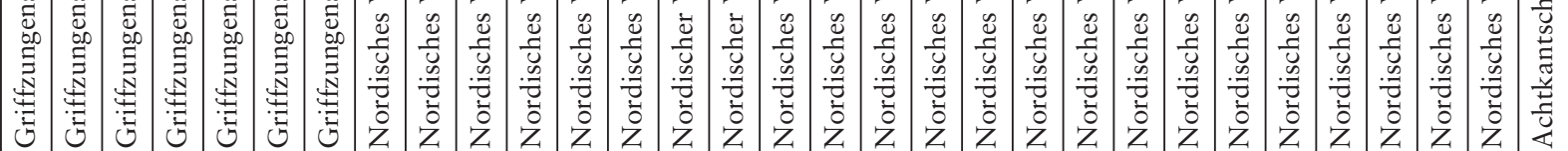

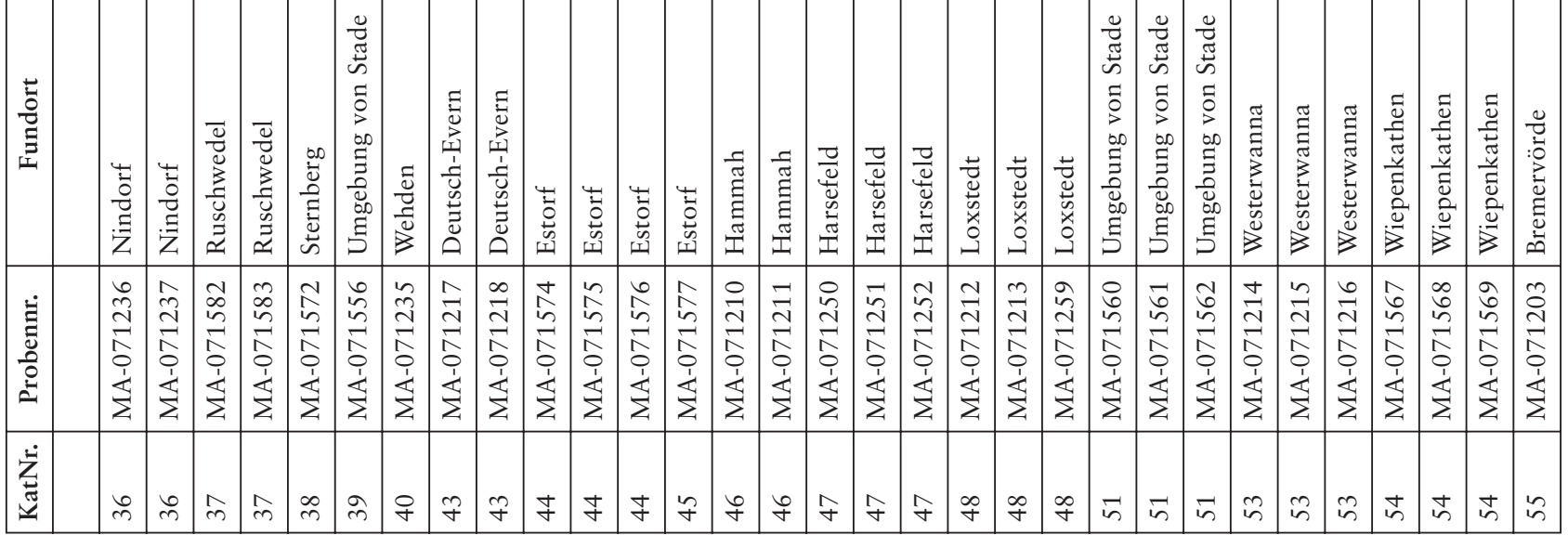




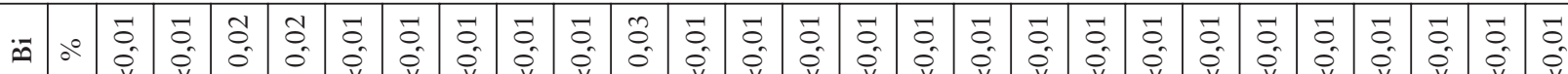

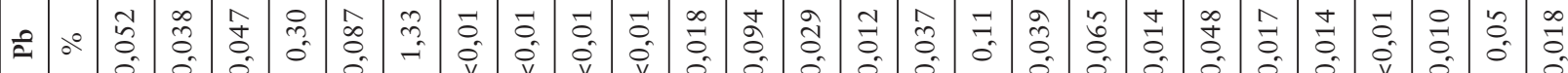

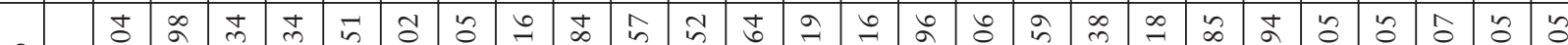

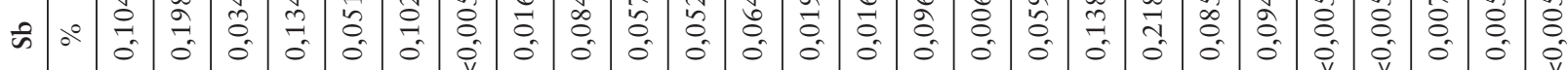

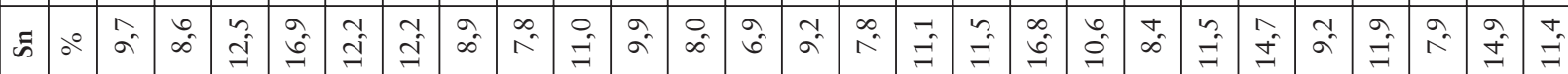
果

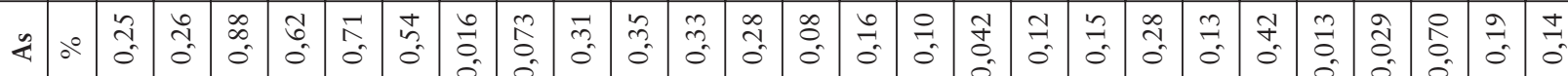

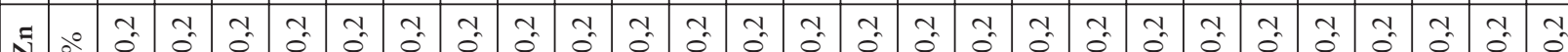

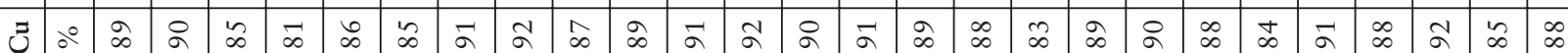
Z

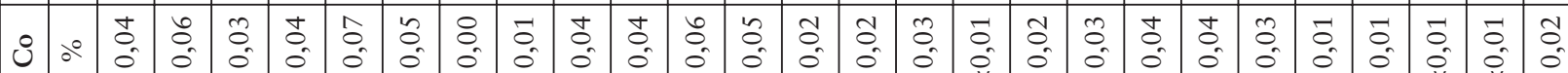

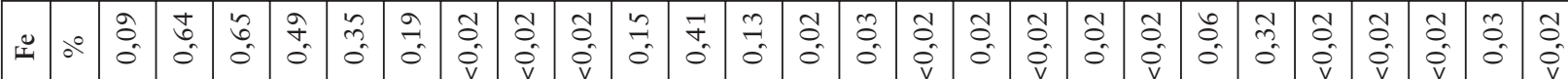

